\title{
DESIGN AND ANALYSIS OF THE IMPACT DIFFUSION HELMET THROUGH A FINITE ELEMENT ANALYSIS APPROACH.
}

\author{
A Thesis \\ presented to \\ the Faculty of California Polytechnic State University, \\ San Luis Obispo
}

\author{
In Partial Fulfillment \\ of the Requirements for the Degree \\ Master of Science in Mechanical Engineering
}

by

Steven Paul Warnert

October 2016 
(C) 2016

Steven Paul Warnert

ALL RIGHTS RESERVED 
COMMITTEE MEMBERSHIP

TITLE: Design and Analysis of the Impact Diffusion Helmet through a Finite Element Analysis Approach

AUTHOR: Steven Paul Warnert

DATE SUBMITTED: October 2016

COMMITTEE CHAIR: Peter Schuster, Ph.D.

Professor of Mechanical Engineering

COMMITTEE MEMBER: Joseph Mello, Ph.D.

Professor of Mechanical Engineering

COMMITTEE MEMBER: Brian Self, Ph.D.

Professor of Mechanical Engineering 


\begin{abstract}
Design and Analysis of the Impact Diffusion Helmet through a Finite Element Analysis Approach
\end{abstract}

Steven Paul Warnert

By applying the finite element approach to the design and analysis of the impact diffusion helmet, many helmet configurations were able to be analyzed. Initially it was important to determine what design variables had an influence on the impact reducing abilities of the helmet design. The helmet was run through a series of Abaqus simulations that determined that a design with two oval shaped channels running along the length of the helmet was best. Next, these options were optimized to generate the helmet that produced the greatest impact reduction. The optimization simulations determined that a helmet that pushed the channels as far from the impact zone as possible reported the lowest acceleration. This indicated that removing the channels from play was most advantageous from an impact reduction perspective. Finally, a 3-D printed experimental helmet was impact tested and compared to a 3-D printed control helmet. The experimental helmet brought the channels back into the impact zone in order to judge if they had a physical effect on the acceleration. Both the simulations and the subsequent physical testing indicated that the Impact Diffusion Helmet design has a negative influence on the concussion reducing properties of a football helmet. 


\section{ACKNOWLEDGMENTS}

I had a lot of help and support on this project. First of all, I'd like to thank Michael

Schuster. His expertise and knowledge were an invaluable aid to my research and having someone to work through the challenges of a thesis made the work much more manageable.

Thanks also to Dr. Schuster for his work getting the project going and keeping it moving forward. His guiding hand provided me with many suggestions to keep the project on track and how to best proceed when obstacles were encountered. His flexibility and willingness to talk about even the smallest problem was very much appreciated.

I'd like to acknowledge Dr. Mello and Dr. Self for being on my committee. Their commentary was another great thing to have.

To Brad and Alicia, thank you for bring this project to Cal Poly and allowing me to work on it with you.

I'd also like to thank my friends and family for their unwavering support in my most stressful states. My girlfriend, Hannah Wagner, faced most of this and responded with love and understanding. 


\section{TABLE OF CONTENTS}

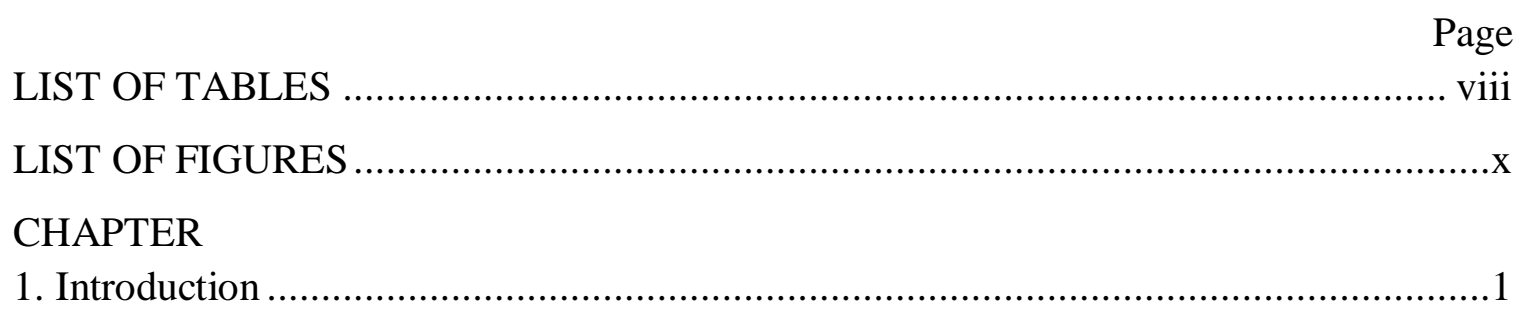

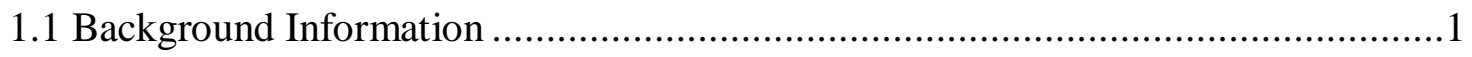

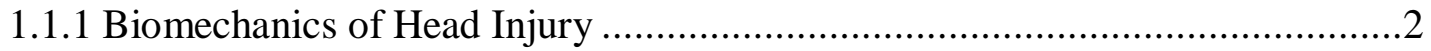

1.1.2 Football Helmet Standards and Test Equipment .......................................5

1.1.3 Effect of Peak Acceleration .................................................................. 8

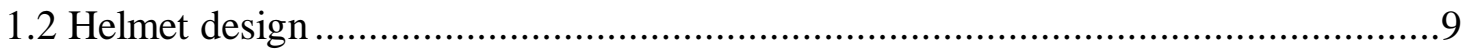

1.2.1 Traditional Football Helmets .................................................................. 9

1.2.2 Proposed Helmet Design ....................................................................... 10

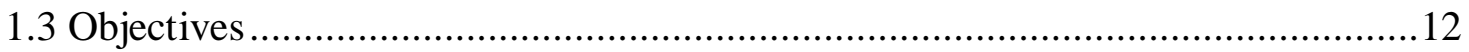

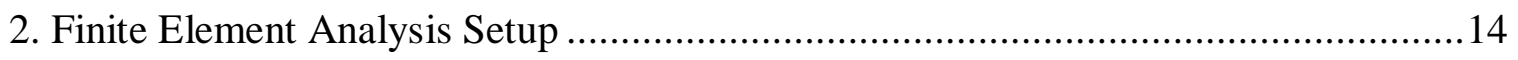

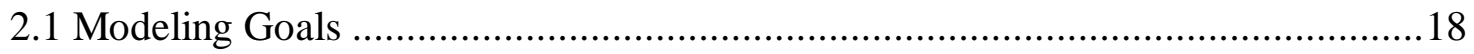

2.2 Model Development .......................................................................... 18

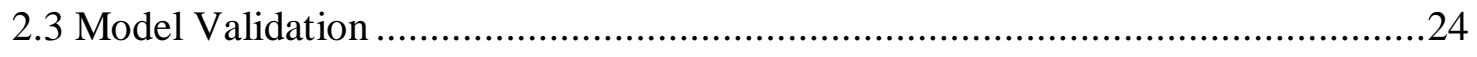

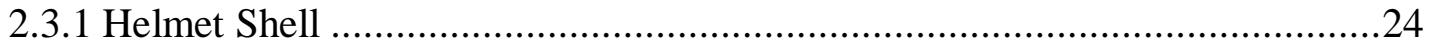

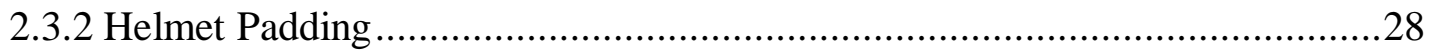

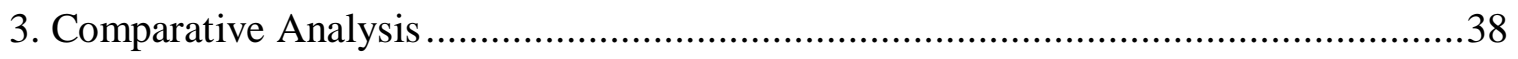

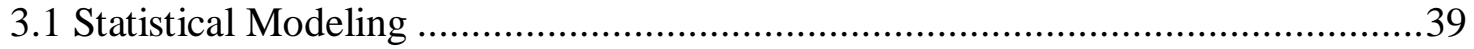

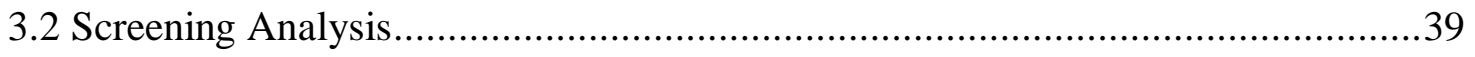

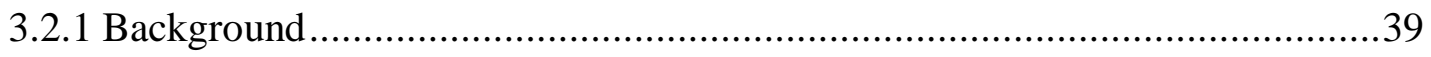

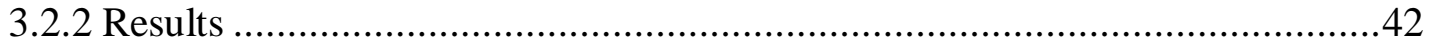

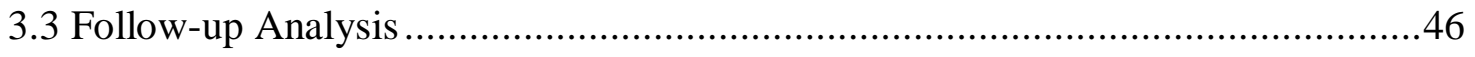

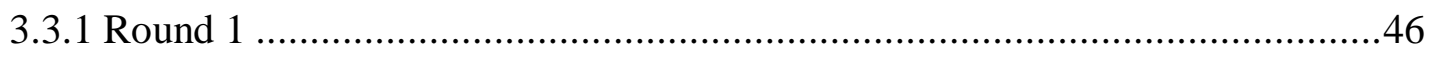

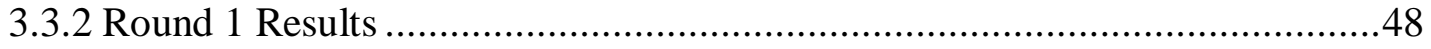

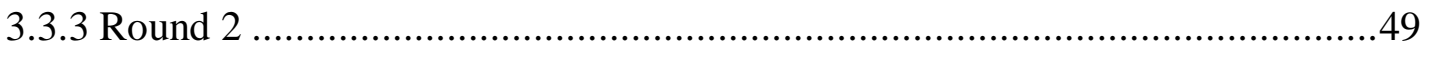

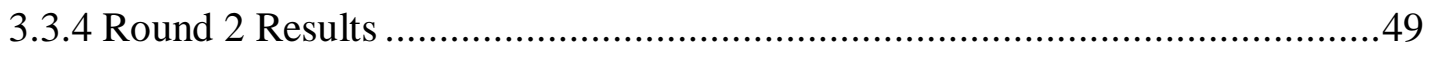




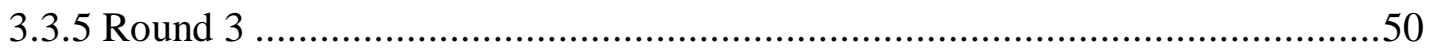

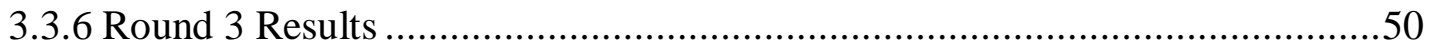

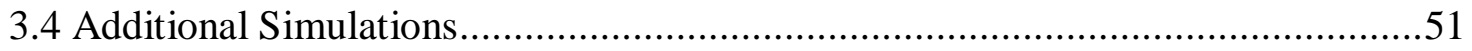

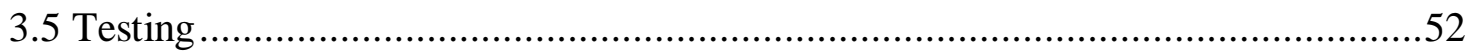

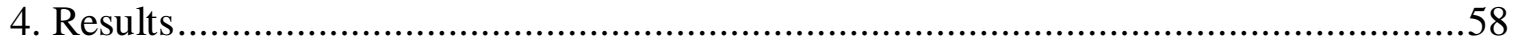

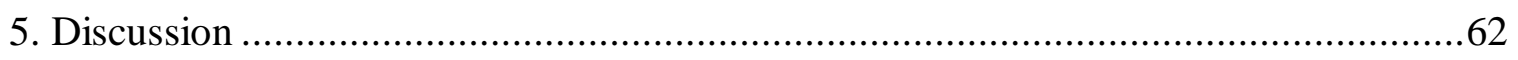

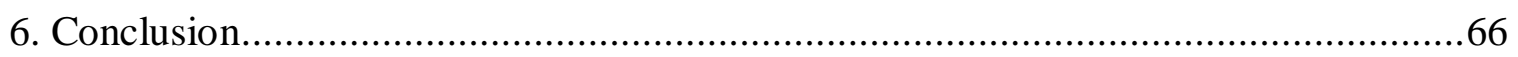

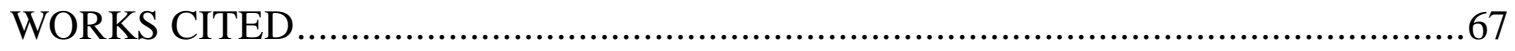

APPENDICES

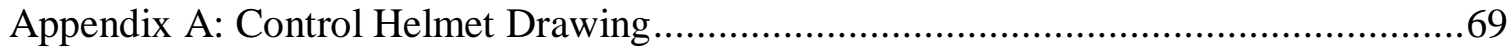

Appendix B: Properties used in Abaqus/CAE simulations.........................................70

Appendix C: Max Bending and Spherical Stress Matlab Code .....................................73

Appendix D: Thermoplastic Polyurethane Material Properties ...................................74

Appendix E: Low Density Foam Material Properties ..............................................75

Appendix F: Drawing of Representative Experimental Model.....................................76

Appendix G: Representative Matlab Code for Calculating Severity Index ....................77

Appendix H: Drawing of the Optimal Helmet Configuration ....................................81

Appendix I: High Speed camera screen captures of the first control helmet impact test ..82 


\section{LIST OF TABLES}

Table

Table 1. Element type and seed size for all the components of the Abaqus models.........22

Table 2. Table of $\mathrm{A}, \mathrm{B}$ and $\mathrm{C}$ values with respect to values of mu to calculate

maximum deflection, membrane stress, and bending stress for a partial spherical

shell under loading at one pole. [18]

Table 3. List of factors available to be modified in the design of the impact reduction helmet.

Table 4. Screening ANOVA factors and levels.

Table 5. Run table for a $2{ }_{\text {III }}^{6-3}$ Fractional Factorial ANOVA design.

Table 6. Calculated Severity Index for the screening ANOVA. The control severity indices are also included to allow for easy comparison between the experimental trials.

Table 7. Factors and levels for Follow-up ANOVA study.

Table 8. Run table for the $2^{3-1}$ fractional factorial design for Follow-up ANOVA \#1 analysis.

Table 9. Run table for the $2^{3-1}$ fractional factorial design for Follow-up ANOVA \#2 analysis.

Table 10. Run table for the $2^{3-1}$ fractional factorial design for Follow-up ANOVA \#3 analysis.

Table 11. Run table for a $2^{3-1}$ fractional factorial ANOVA design.

Table 12. Severity Index results from follow-up ANOVAs 1, 2 and 3.

Table 13. Factors and winning levels from follow-up ANOVAs 1, 2 and 3.

Table 14. Run table for the $2^{3-1}$ fractional factorial design for Follow-up ANOVA \#4 analysis.

Table 15. Run table for the $2^{3-1}$ fractional factorial design for Follow-up ANOVA \#5 analysis.

Table 16. Severity Index results from follow-up ANOVAs 4 and 5.

Table 17. Factors and winning levels from follow-up ANOVAs 4 and 5.

Table 18. Run table for the $2^{3-1}$ fractional factorial design for Follow-up ANOVA \#6 analysis. .50

Table 19. Severity Index results from follow-up ANOVA 6.

Table 20. Final Analysis Severity Index results. Includes the optimized design and a three channeled design using the optimized channel geometry.

Table 21. Impact metric values for the 3-D printed control and channeled helmet designs averaged over five runs.

Table 22. Stress and strain data for thermoplastic formed polyurethane at $23^{\circ} \mathrm{C}$ taken from the above plot. 
Table 23. Table representation of the stress and strain data taken from the Ford motor company handbook on impact reducing foams........................................ 75 


\section{LIST OF FIGURES}

Figure

Page

Figure 1. Pie chart indicating the number of concussions reported in a single sports season from "Epidemiology of sports-related concussion in seven US high school and collegiate sports." Football accounts for about four time the next largest

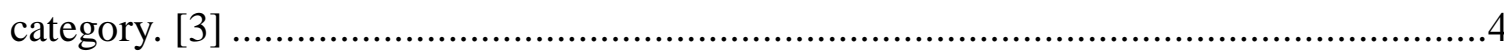

Figure 2. NOCSEA approved helmet testing apparatus [7] ......................................6

Figure 3. All necessary drop criteria to qualify a newly manufactured helmet to NOCSAE standards. [8] ............................................................................. 7

Figure 4. All necessary drop criteria to recertify a football helmet to NOCSAE standards. [9] ............................................................................................

Figure 5. Comparison of injury risk curve of previously published data. Displays head accelerations only associated with concussive impacts. [10] ..............................9

Figure 6. Channels imbedded in helmet shell going from front to back along helmet. .10

Figure 7. Image describing how a waveguide transmits an applied signal along its length.

Figure 8. Solid model of impact reduction helmet design. Note the padding between the channels: the sub-padding. .11

Figure 9. Depiction of multiple material cores.

Figure 10. Simple finite element system with two bar elements and a single degree of freedom per node.

Figure 11. Simple finite element analysis system with two bar elements and two degrees of freedom per node. .15

Figure 12. Deformed bar element shape with linear shape function (left) and quadratic shape function (right) .16

Figure 13. The simple spherical shell model used as the basis for the computer analysis. .20

Figure 14. Half model used in the Abaqus simulations.............................................21

Figure 15. Denotation of the contact method used at each interaction area. .23

Figure 16. Image accompanying equations for a partial spherical shell from Roark's

Formulas for Stress and Strain indicating the variables needed to complete the analysis. [18] .25

Figure 17. Spherical shell validation model..... .26

Figure 18. Mesh convergence plot for max displacement from the spherical shell analysis. .27

Figure 19. Mesh convergence plot for stress from the spherical shell analysis..............28

Figure 20. Schutt Air XP padding. [19]. 
Figure 21. Abaqus model of thermoplastic polyurethane Schutt Air XP padding.

Shown as a section view to display internal features.

Figure 22. Force and displacement data from the Ford handbook (theory) plotted with data from an analogous Abaqus simulation (model).

Figure 23. Foam block test model used to verify that Abaqus was accurately

representing the data from the Ford handbook.

Figure 24. Compressive force over time for different seed values for the foam

validation test. The 0.25 and 0.1 seed size were almost identical so a final seed size of 0.25 was chosen.

Figure 25. Small scale testing configuration and apparatus. The 3-D printed

prototype was taped to the aluminum impactor beneath Schutt Air XP padding.

This assembly was dropped onto the impact surface (blue) and the acceleration of

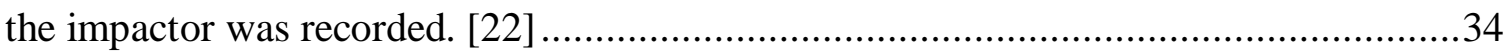

Figure 26. Control model used to validate padding properties. ..................................36

Figure 27. Channeled model used in validating padding properties. .............................36

Figure 28. Acceleration pulse from the control prototype simulation............................37

Figure 29. Acceleration pulse from the control physical testing impact. .........................37

Figure 30. Representative Abaqus model for the analysis performed. The changes

between the runs of the analysis involved changing the geometry of the helmet

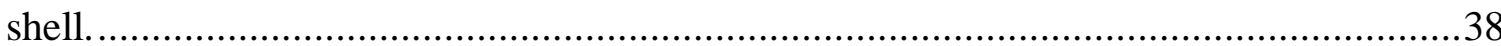

Figure 31. Channels spaces evenly in a 90 degree wedge......................................42

Figure 32. Main effects plot generated for the screening ANOVA. .............................43

Figure 33. Raw (unfiltered) acceleration data from Abaqus/CAE for Screening

ANOVA Run 1.

Figure 34. Acceleration data from Screening ANOVA Run 1 filtered with 4-pole

low pass Butterworth filter. The acceleration data is cut off at $4 \mathrm{G}$ 's to comply with

NOCSAE Severity Index calculation standards.............................................45

Figure 35. Rendering of the optimal configuration of the impact reduction helmet.........51

Figure 36. Impact testing machine used to test the 3-D printed control and channeled helmets. [22] ......................................................................................53

Figure 37. Outer side of the control and channeled 3-D printed football helmets.

Both designs utilized the same outer geometries.

Figure 38. 3-D printed control football helmet used to compare against the channeled design. 55

Figure 39. 3-D printed impact diffusion helmet design. .55

Figure 40. Plot of the filtered acceleration pulse from the third drop of the control helmet. This plot shows the entire duration of the impact that exists above $4 \mathrm{G}$ 's. Red line indicates where contact with impact pad ended. 
Figure 41. Plot of the filtered acceleration data from the third drop of the control helmet. This plot only shows up to $25 \mathrm{~ms}$ pulse time since that was the duration of impact determined through studying the high speed camera footage.

Figure 42. Graphical representation of stress and strain data for thermoplastic formed polyurethane. The data curve used is the one at $23^{\circ} \mathrm{C}$ 74

Figure 43. Stress strain data from the Ford motor company handbook on impact reducing foams. The important curve here is the "Stress" curve in blue. .75 


\section{Introduction}

With concussions becoming a much more significant issue in the faster paced and harder hitting sports of today, there is a strong movement behind further protecting the players from serious and long term health problems. Brad Bartholomay came to the Mechanical Engineering Department at Cal Poly San Luis Obispo with an idea for a new design of football helmets that could significantly reduce the chance of concussion in impact scenarios. The project was conducted under the guidance of Cal Poly professor Peter Schuster.

The overarching goal of this project was to model Brad's design for the impact diffusion helmet in Finite Element Analysis (FEA) software and run it through numerous analyses to determine the viability of the design. Further analysis in the FEA software determined the most effective geometries to reduce the impact to the player's head. Once the most effectual design was established, a prototype of the design was produced to physically validate the FEA results.

\subsection{Background Information}

The most fundamental aim of the project was to determine if the impact diffusion helmet design sufficiently reduces the chance of concussion. In order to gain a clearer understanding of how the helmet will have to behave and if this concept will in fact diffuse energy away from the athlete's head, a variety of relevant information was compiled and analyzed. 


\subsubsection{Biomechanics of Head Injury}

Actual concussions are diagnosed in a variety of ways, but the mode through which a concussion happens is consistent in every case. Concussions occur in a scenario in which the body experiences a blow that causes the head to be thrown back and forth. $[1,2]$ This force is transmitted to the cerebral tissue which jostles the brain back and forth or side to side within the skull. The resulting trauma from stretched and damaged brain cells is considered a concussion. Additionally, while concussions are most closely linked to impact situations, it is also not uncommon for a concussion to occur from whiplash or a similar non-contact event. Short-term symptoms of a concussion can include: one pupil dilating more than the other, drowsiness, headache, nausea, and loss of consciousness.

Unfortunately, accurately diagnosing a concussion can be extremely difficult. With only 200,000 emergency department visits annually for concussions, it is clear to researchers that the vast majority of head injuries are treated on site or go undiagnosed. [3] They even go so far as to speculate that the actual number of sports-related concussions seen annually could be nearer to 3.8 million. [4] This makes traumatic brain injuries a much larger problem than it would seem at first glance.

There are many existing methods to diagnose a concussion. Just in the last few years researchers have begun to consider peak rotational acceleration as a cause of concussion as much as peak linear acceleration. In 2013, a composite concussion risk criteria analysis was published that accounted for the presence of both linear and rotational accelerations. [4] While this was not the first composite analysis produced, it provided a 
further step into diagnosing concussions using actual impact data. The team developed a set of compound curves that judged concussion risk based on the peak accelerations experienced using data taken from acceleration telemetry during actual real-world impacts from the Head Impact Telemetry System (HITS), the National Football League (NFL) and lab reproduced impacts. They determined that this multipart accounting was significantly better at determining if a concussion had in fact occurred. Despite this increased chance of diagnosing a concussion if the player is wearing acceleration telemetry equipment, the diagnosis of concussions with no physical data is still very subjective.

In a study for the Concussion Prevention Initiative, athletic trainers at seven schools were tasked with identifying and grading concussions based on symptoms identified by the trainers. [3] The athlete had to exhibit one or more cognitive (headache, balance problems, dizziness, nausea, blurred vision, numbness, tingling, vomiting, drowsiness, fatigue, sensitivity to light, sensitivity to noise), neurobehavioral (sleeping more than usual, trouble sleeping irritability, sadness), or somatic (feeling "in a fog", difficulty concentrating, difficulty remembering) symptoms to be considered a concussion. The fact that any of these symptoms could be experienced without the help of an accompanying concussion made the diagnosis that much more difficult. If a concussion was detected, the athletic trainers then graded the concussion's symptoms on a sliding scale from 0 to 6 and identified the mechanisms by which the concussion occurred on a standardized form called the "concussion index". The data were then compiled to determine the prevalence 
and severity of concussions in seven sports. Football had the highest rate of concussions by a factor of four. (Figure 1)

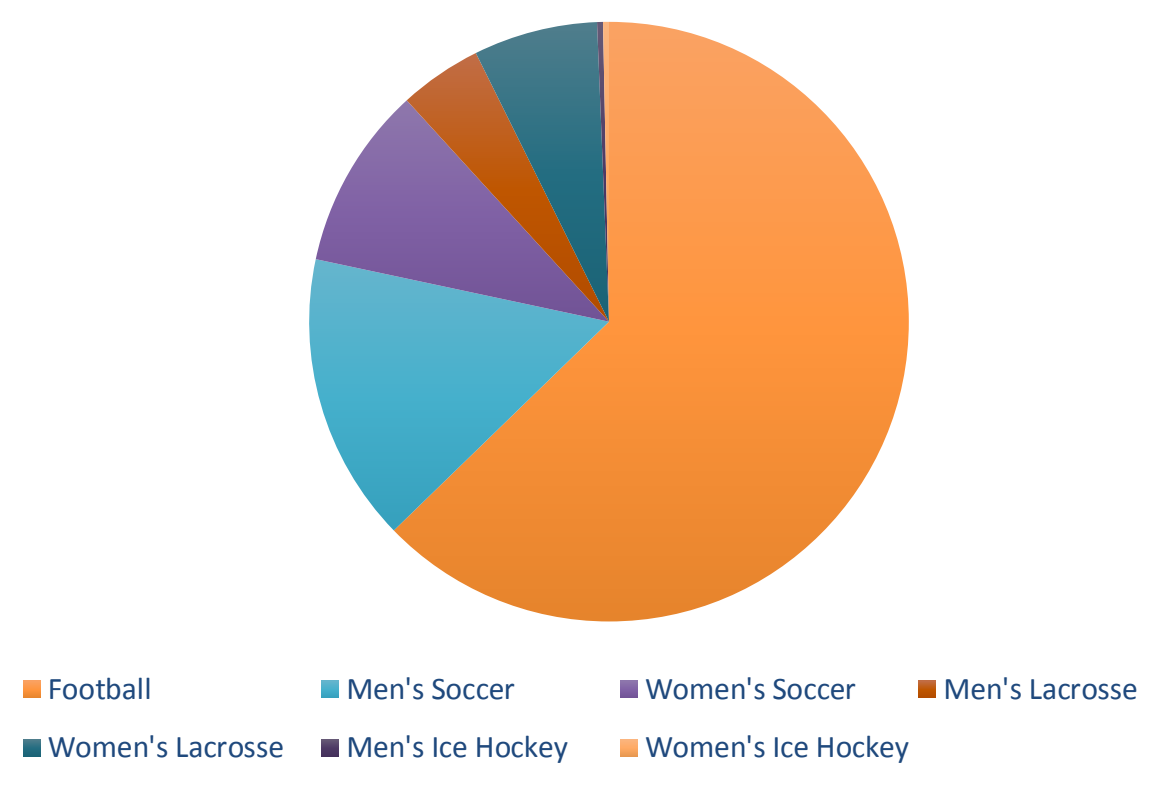

Figure 1. Pie chart indicating the number of concussions reported in a single sports season from "Epidemiology of sports-related concussion in seven US high school and collegiate sports." Football accounts for about four time the next largest category. [3]

A recent topic of intense debate has been whether the culmination of hundreds of small impacts will cause long term head trauma. In March of 2016 the NFL announced the long term implications of repeated impacts to the head. [5] Based on the work of Dr. Ann McKee, a Boston University neuropathologist, chronic traumatic encephalopathy (CTE) was found to be extremely prevalent in football players. She has diagnosed CTE in 90 of 94 former NFL players she examined. It can no longer be suggested that small impacts have no effect on later brain injury and any new technology to help mitigate these impacts would be very well received. 


\subsubsection{Football Helmet Standards and Test Equipment}

Throughout the history of sports there have been a number of equipment standards that athletic programs have used to design and maintain their equipment. Despite having once come from the American Society for Testing and Materials (ASTM) and the International Standards Organization (ISO), the current accepted standard set comes from the National Operating Committee on Standards for Athletic Equipment (NOCSAE).[6] The NOCSAE standards do reference many ANSI and ISO standards, however, so all the standards organizations are tightly interwoven.

Each individual football helmet must be rigorously tested before it's allowed out onto the field. [7, 8] A NOCSAE testing apparatus is one way for appropriately determining whether or not the helmet passes or fails the test. It consists of a large frame that holds a simulated head off the ground, which is then hoisted to a height that will achieve $11.34 \mathrm{ft} / \mathrm{s}, 13.89 \mathrm{ft} / \mathrm{s}, 16.04 \mathrm{ft} / \mathrm{s}$, and $17.94 \mathrm{ft} / \mathrm{s}$ drop speeds and is allowed to fall and strike a rubber pad. These speeds roughly correspond to $2 \mathrm{ft}, 3 \mathrm{ft}, 4 \mathrm{ft}$, and $5 \mathrm{ft}$ drops. A multi-axial accelerometer captures the acceleration data during the event. The testing apparatus can be seen in Figure 2 below. In order to most accurately test a helmet's ability to perform up to specification, NOCSAE adopted their Severity Index (SI) criterion. The Severity Index is determined through a simple impact equation seen in Equation 1 below. 


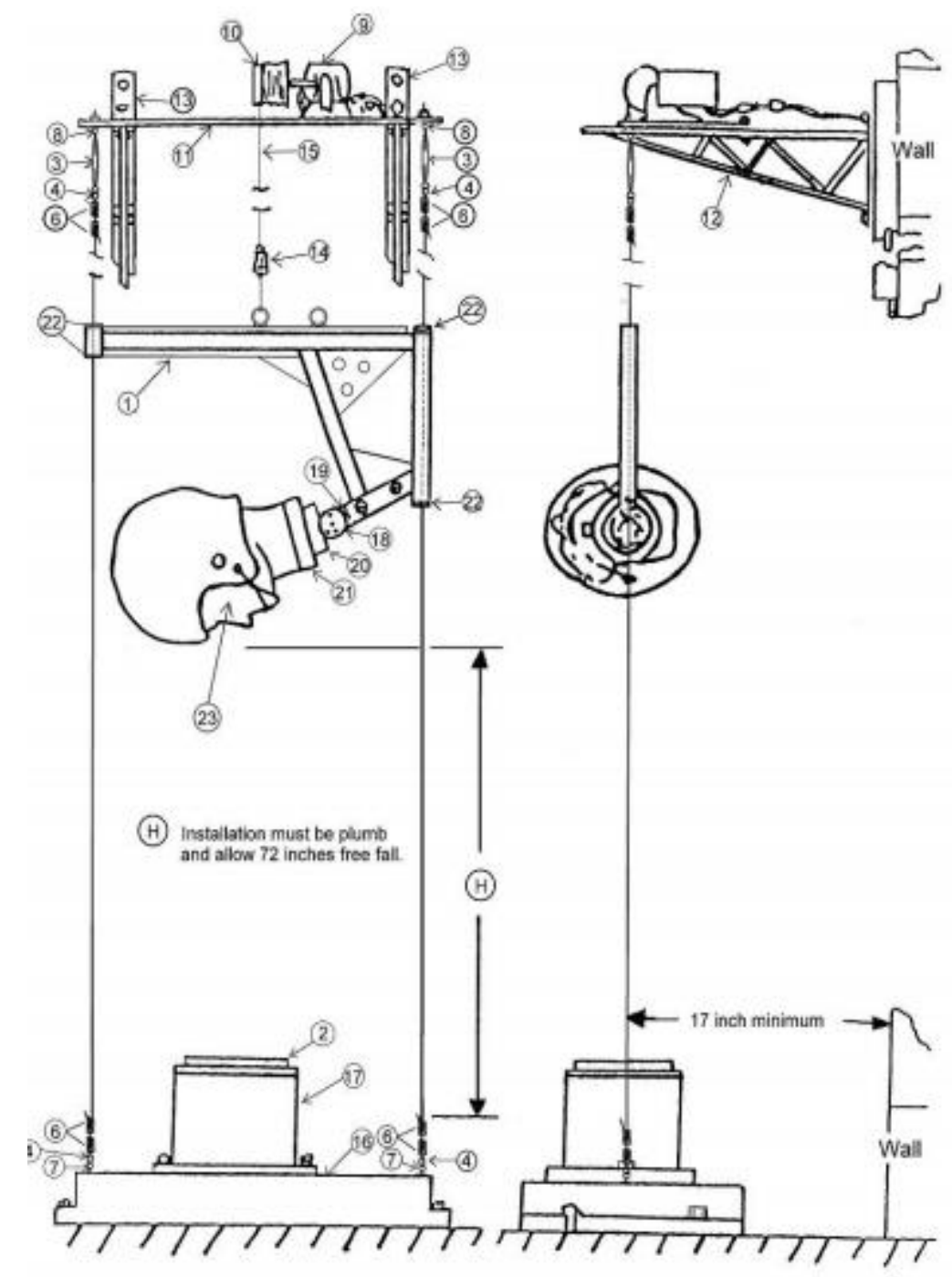

Figure 2. NOCSEA approved helmet testing apparatus [7]

$$
S I=\int_{0}^{T} A^{2.5} d t
$$

In this expression, SI is the calculated Severity Index, $\mathrm{T}$ is the total duration of the impact, and $\mathrm{A}$ is the acceleration seen by the accelerometer. [7] The duration of the impact is determined electronically from the time that the acceleration signal rises above $4 \mathrm{~g}$ 's to when it falls back below $4 \mathrm{~g}$ 's. The SI is determined for impacts at room and elevated temperatures at the front, side, front boss, rear boss, rear, top, and a random spot 
on the helmet. The testing table can be seen below in Figure 3. The regulating body determined that if the Severity Index of any impact did not exceed 1200 SI and the Severity Index of the test performed at $11.34 \mathrm{ft} / \mathrm{s}$ did not exceed $300 \mathrm{SI}$, then the helmet would pass and could be worn safely.

LOCATION - DROP velocities - ft/s (m/s)
(All drop velocities must be within +3\% -0\%)
\begin{tabular}{|c|c|c|c|c|c|c|c|}
\hline \multirow{4}{*}{$\begin{array}{c}\text { Ambient } \\
\text { Temperature }\end{array}$} & FRONT & SIDE & F. BOSS & R. BOSS & REAR & TOP & RANDOM \\
\cline { 2 - 9 } & $11.34(3.46)$ & $11.34(3.46)$ & $11.34(3.46)$ & $11.34(3.46)$ & $11.34(3.46)$ & $11.34(3.46)$ & $11.34(3.46)$ \\
\cline { 2 - 9 } & $13.89(4.23)$ & $13.89(4.23)$ & & & & & \\
\cline { 2 - 9 } & $16.04(4.88)$ & $16.04(4.88)$ & & & & & \\
\cline { 2 - 9 } & $17.94(5.46)$ & $17.94(5.46)$ & $17.94(5.46)$ & $17.94(5.46)$ & $17.94(5.46)$ & $17.94(5.46)$ & $17.94(5.46)$ \\
\hline \hline \multirow{3}{*}{$\begin{array}{c}\text { High } \\
\text { Temperature }\end{array}$} & $17.94(5.46)$ & $17.94(5.46)$ & $17.94(5.46)$ & $17.94(5.46)$ & $17.94(5.46)$ & $17.94(5.46)$ & $17.94(5.46)$ \\
\cline { 2 - 9 } & $17.94(5.46)$ & $17.94(5.46)$ & $17.94(5.46)$ & $17.94(5.46)$ & $17.94(5.46)$ & $17.94(5.46)$ & $17.94(5.46)$ \\
\hline \hline
\end{tabular}

Figure 3. All necessary drop criteria to qualify a newly manufactured helmet to NOCSAE standards. [8]

There is a slightly different standard that NOCSAE publishes that considers recertifying football helmets as opposed to certifying newly manufactured helmets. [9] The testing apparatus here is the same as the full certification but it is run through significantly fewer tests. It is only dropped to achieve the $17.94 \mathrm{ft} / \mathrm{s}$ speed at impact and only impacts the front, side, rear, and top. The testing table can be seen in Figure 4 below. The committee determined that if the SI achieved by each impact did not exceed 1200 SI for a helmet manufactured after January 1, 1997 or 1500 SI for a helmet manufactured before January 1, 1997 the helmet would pass the certification. 
LOCATION - DROP velocities $-\mathrm{ft} / \mathrm{s}(\mathrm{m} / \mathrm{s})$

(All drop velocities must be within $+3 \%-0 \%$ )

\begin{tabular}{||c|c|c|c|c||}
\hline \hline \multirow{4}{*}{$\begin{array}{c}\text { Ambient } \\
\text { Temperature }\end{array}$} & $\begin{array}{c}\mathbf{( 1 )} \\
\text { FRONT }\end{array}$ & $\begin{array}{c}\mathbf{( 2 )} \\
\text { SIDE }\end{array}$ & $\begin{array}{c}\mathbf{( 5 )} \\
\text { REAR }\end{array}$ & $\begin{array}{c}\text { (6) } \\
\text { TOP }\end{array}$ \\
\cline { 2 - 6 } & $17.94(5.46)$ & $17.94(5.46)$ & $17.94(5.46)$ & $17.94(5.46)$ \\
\cline { 2 - 6 } & $17.94)$ & $17.94(5.46)$ & $17.94(5.46)$ & $17.94(5.46)$ \\
\hline
\end{tabular}

Figure 4. All necessary drop criteria to recertify a football helmet to NOCSAE standards. [9]

\subsubsection{Effect of Peak Acceleration}

While current football helmets are designed specifically to prevent skull fracture, there does seem to be a correlation between lowering the Severity Index and lowering the incidence chance of concussion. [10] Using a statistical method named the Summation of Tests for the Analysis of Risk (STAR), researchers were able to develop a concussion incidence curve that associated the Severity Index from NOCSAE and the peak acceleration in the impact. The incidence curve can be found below in Figure 5. While there is a large spread between the results from the different sources, the shape of the regression is consistent. It is clear that the lower the peak acceleration, and consequently the lower the SI, the lower the risk of concussion. It is worth noting that the researchers only considered linear acceleration in their calculations, so this data may not be $100 \%$ applicable due it neglecting the rotational component of acceleration. Despite this, rotational and linear components of acceleration are closely related in an impact scenario, so the rotational acceleration will likely follow the linear acceleration. Because this study was mostly determining if lowering peak acceleration reduced concussion chance, it is still appropriate to consider these results. 


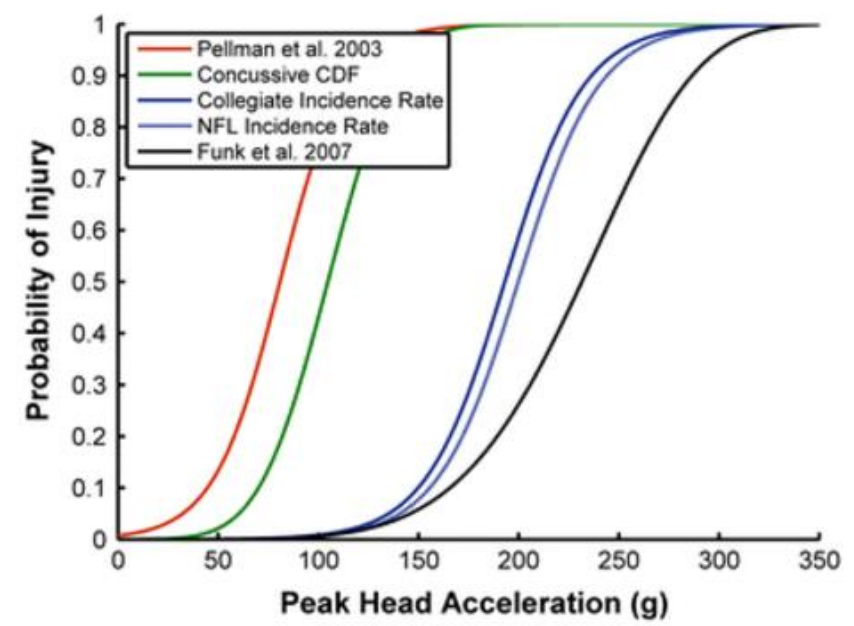

Figure 5. Comparison of injury risk curve of previously published data. Displays head accelerations only associated with concussive impacts. [10]

\subsection{Helmet design}

This project surrounds the testing and development of a completely new impact diffusing design for the hard shell of a football helmet. The helmet is comprised of several key geometric features that give it its unique impact reducing properties.

\subsubsection{Traditional Football Helmets}

It's important to consider the design of traditional football helmet to appreciate the changes made to the impact diffusion helmet. Conventional helmets consist of polycarbonate shell surrounding specially-designed helmet padding. [11] Polycarbonate is chosen for its supreme impact resistance and light weight. Frequently, air filled padding is also used to provide a custom fit for the players. In between the helmet padding and the player's head, many manufacturers include a comfort foam which does little for impact attenuation, but greatly increases the comfort of the helmet. 


\subsubsection{Proposed Helmet Design}

The new football helmet consists of a number of key attributes. The first and most important of these features is the concept of hollow channels imbedded in the helmet shell running lengthwise from front to back. This idea is visualized in Figure 6 . The channels are meant to contain and funnel a portion of the impact energy towards the openings on either end of the channel and release that energy without it moving through the padding and into the head. There is, however, no physical basis for this kind of energy transference. The closest analogue to the channels is a structure called a waveguide that directs sound or other signals through a duct or other similar geometry. $[12,13]$ This works only by introducing a signal to one end of the waveguide and rebounding the signal along the walls to the other side. (Figure 7). The phenomenon is not extended to a signal entering through the side of the guide. It is possible, however, that this mechanism or another unknown mechanism will apply to the helmet, even though there is very little literature regarding the specifics of this scenario.

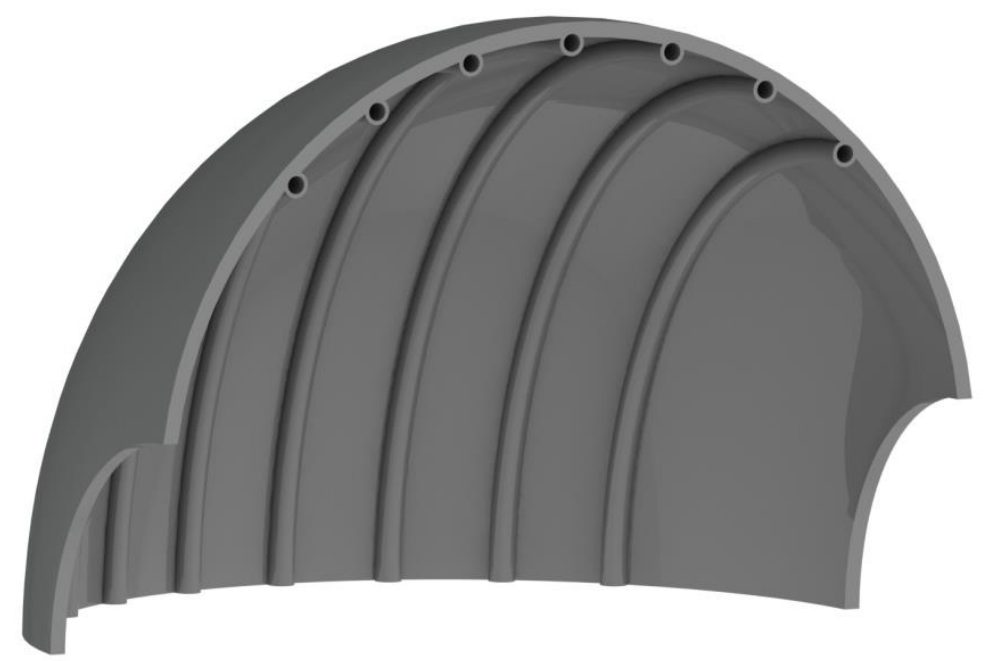

Figure 6. Channels imbedded in helmet shell going from front to back along helmet. 


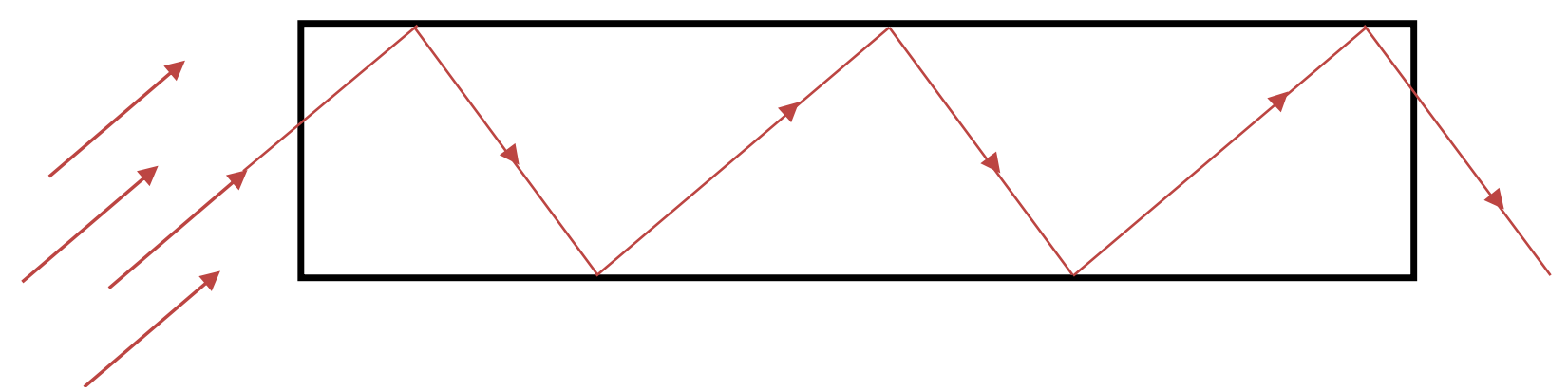

Figure 7. Image describing how a waveguide transmits an applied signal along its length.

In addition to traditional helmet padding, there will be padding between the channels to eliminate any empty space between the helmet padding and the shell. This padding is called the "sub-padding" and is denoted in Figure 8.

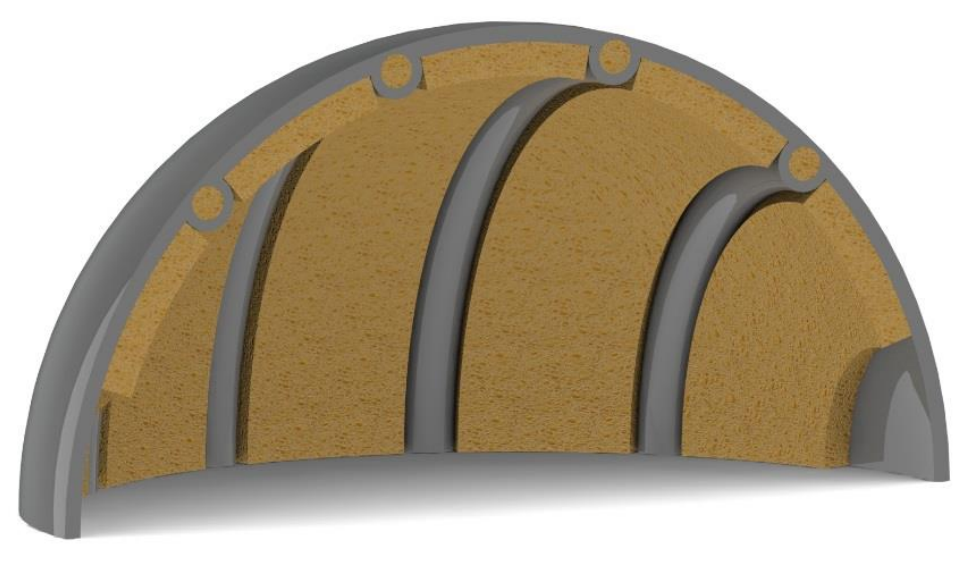

Figure 8. Solid model of impact reduction helmet design. Note the padding between the channels: the sub-padding.

Secondly, each of these channels can be filled with a core material, also shown in Figure

8. This core material is meant to help capture and transmit the energy from the impact. It was further proposed that having multiple core materials, a more rigid center core surrounded by a coating of a less dense substance, could help the performance of the helmet. The center core would be there to transmit the vibration along the channel while 
the coating would help with damping the impact. Figure 9 shows an example of multimaterial cores.

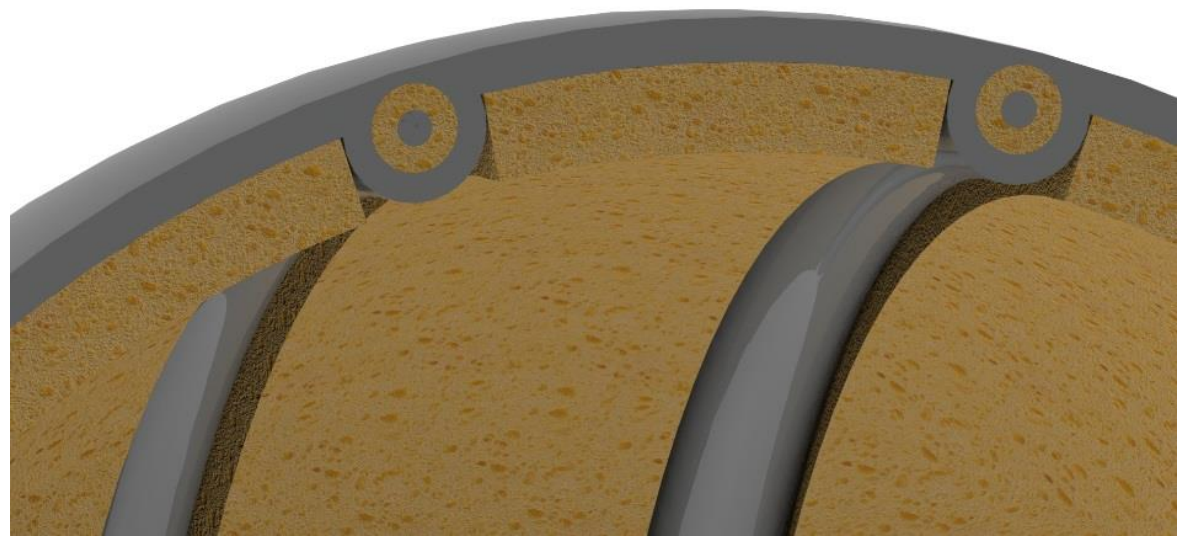

Figure 9. Depiction of multiple material cores.

Aside from the channels and the cores, many of the specifics of the design were yet to be determined. The relative sizes of the channels, the core material, the shape of the channels, the spacing of the channels, and how many channels will be on the helmet were all determined through rigorous computer analysis of various design configurations.

\subsection{Objectives}

The objectives for this project were separated into several major subgroups. This first was to verify the physical testing by simulating it in the finite element software Abaqus. By matching the results from the physical testing, the parameters that those simulations used were authenticated. This allowed them to be confidently used later in more extensive models. Once the model parameters were established, the final design of the impact diffusion helmet was determined. A series of simulations with the new design configuration were run to determine if any of the design variables had a significant impact on reducing the acceleration of the head. The results of this screening test were then input into a second more extensive analysis to optimize the chosen variables for 
impact reduction. Once an optimal configuration had been established, a 3-D printed version was physically tested and compared to a control helmet to gauge real-world effect. A final simulation was then run to compare to the final physical test and used to validate the parameters used in the optimization trials. 


\section{Finite Element Analysis Setup}

Due to complicated nature of the design, the biggest problem was determining how to establish the validity of the proposed design and then how to best optimize the design geometries. Hand calculations were quickly ruled out due to complexity and lack of constitutive models. Physical testing was the logical next step, however, it requires a prototype for each design iteration as well as the necessary equipment to accurately capture acceleration data. To allay these concerns, a computer simulation approach was chosen to analyze different configurations of the impact diffusion helmet design.

One of the most advanced methods of virtually simulating physical models is Finite Element Analysis (FEA). This method discretizes each component of the solid model into thousands of nodes, or locations, at which the simulation calculates the required parameters. Adjacent nodes are combined into elements which are given the mechanical properties of the component to be studied.

In its simplest state, an FEA problem can be solved by hand. An example of a situation of this nature can be seen in Figure 10 which depicts a cantilever beam. Here there are only three nodes combined into two elements. We could further split this structure into more elements, adding more nodes and increasing the accuracy of whatever calculation needed to be performed. 


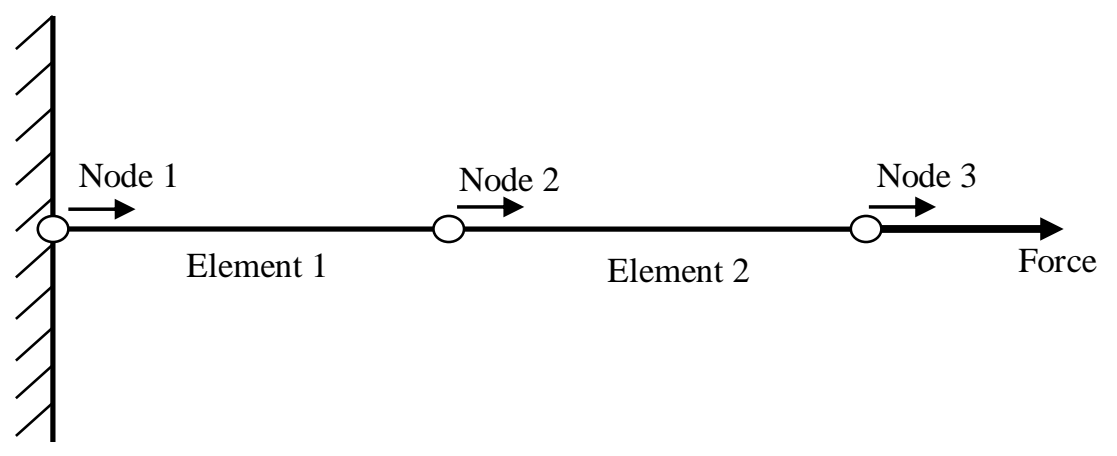

Figure 10. Simple finite element system with two bar elements and a single degree of freedom per node.

For a structural problem like the one above, the displacements at each node are the variables of interest and represent the degrees of freedom (DOF) of the system. Increasing the number of nodes increases the number of DOF allowing the analyst to observe the value of the field variable at more places in the body. The case above has DOF only in the $\mathrm{x}$-direction because the force only acts it the x-direction so there are only displacements in that direction. Figure 11 below adds a DOF in the y-direction to capture the effect on displacement that the additional downwards force produces. In addition to displacement DOF, FEA can also capture rotational degrees of freedom, so a single node can have as many as $6 \mathrm{DOF}$ in a complex scenario.

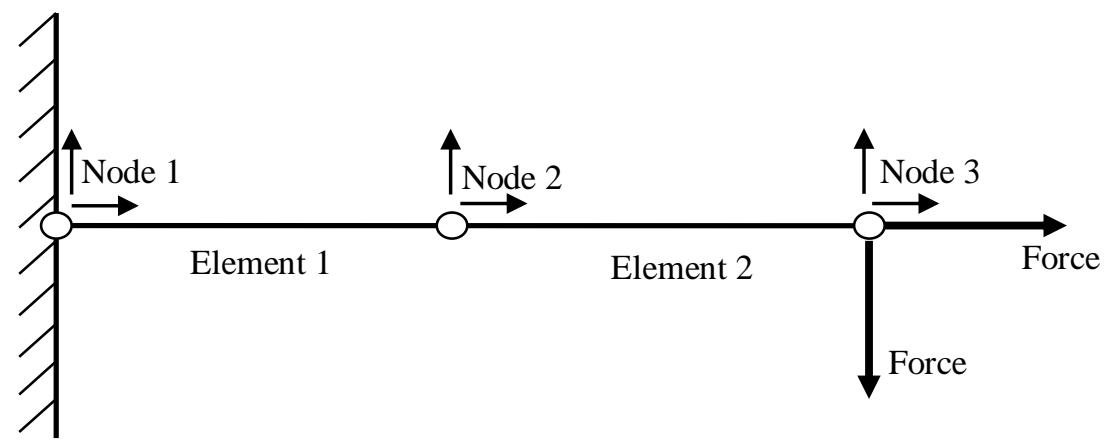

Figure 11. Simple finite element analysis system with two bar elements and two degrees of freedom per node. 
From an accuracy standpoint, the degree to which the displacement varies throughout an element is also very important. For example, if the displacement were to only vary linearly in an element, you would only see a displacement like case 1 in Figure 12. Were the displacement to vary quadratically, however, the element could look something like case 2 in its deformed state. This factor is called the shape function and it determines how the field variable, in this case displacement, varies throughout an element.

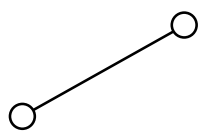

Case 1

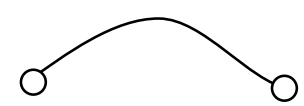

Case 2

Figure 12. Deformed bar element shape with linear shape function (left) and quadratic shape function (right)

Using the minimum potential energy theory, which states that an object will more likely occupy its state of lowest potential energy, the governing equations for FEA can be derived, where $\{F\}$ is the global force vector (the forces acting on every node), $[K]$ is the stiffness matrix, and $\{u\}$ is the DOF vector (the displacements at every node). The stiffness integral, Equation 4, includes [B], which is the derivative of the shape functions, and $[\mathrm{D}]$, which is a material property matrix.

$$
\begin{gathered}
\{F\}=[K]\{u\} \\
\{u\}=\{F\}[K]^{-1} \\
K=\int[B]^{T}[D][B] d V
\end{gathered}
$$


Once the force and stiffness are determined at every node, it is necessary to combine them in the global force vector and global stiffness matrix, respectively. Using these, it is then possible to calculate the global displacement vector using Equation 3. With the DOF vector in hand, it is a simple matter of applying it to Equations 5 and 6 to calculate the strain and stress in each element.

$$
\begin{gathered}
\{\epsilon\}=[B]\{u\} \\
\{\sigma\}=[D]\{\epsilon\}=[D][B]\{u\}
\end{gathered}
$$

While this may not be a big job to do by hand if there are only two of three elements, it becomes a much larger undertaking when analyzing full systems. If a model were to have 10,000 nodes, the global stiffness matrix would become a 10,000 x 10,000 matrix that would then have to be inverted. This is why computers are key to solving FEA problems. Even for fast computers, the inversion of such large matrices can be very time consuming. This is especially the case in any kind of dynamic FEA analysis. The computer will solve the full finite element problem for the first time step then advance to the next, sometimes less than a millisecond, and perform all the calculations again. This provides a series of solutions that can be rendered and viewed in the graphical interface of most FE software. From this interface, other outputs such as velocities and accelerations are able to be viewed for specific points over time. This dynamic method of analysis can be particularly powerful for short duration events like impact since the computation times are relatively short. 


\subsection{Modeling Goals}

Knowing the fundamentals of FEA is essential to developing an accurate model. It allows the user to understand the limitations of the software and how best to employ its strengths. Since this design problem was about the behavior of a helmet in an impact scenario, it was necessary to run the simulation dynamically. This means that instead of defining forces loading a structure and achieving a single result for the deflection and stress state, this method will take prescribed boundary conditions, like an initial helmet velocity, and simulate an entire impact event. The resulting data will include stresses and deflection for all body members, and, more importantly, accelerations. These accelerations will tell us if the impact diffusion design has a lower Severity Index, the metric for concussion risk, than the acceleration data from a control simulation.

Due to the fact that many of the design geometries were determined by the analysis, many simulations had to be run to capture as many design configurations as possible. This meant having an easily adjustable model and a relatively quick computation time.

\subsection{Model Development}

The development of a testing model was the greatest challenge of the project. The simulation went through many iterations before a final set of conditions was established that gave consistent results. Abaqus/CAE version 6.14-2 was used to carry out all the simulations. [14]

The proposed design had been previously tested by a small group of Industrial Engineering students at Cal Poly for their senior capstone project. [15] This was done 
through physical testing of a small 3-D printed prototype. This initial prototype model was the first item that was simulated in Abaqus and much of the preliminary modeling was done by graduate student Michael Schuster. This simulation was made primarily to identify the key challenges that would present themselves when simulating a larger model, but this early simulation also became instrumental in verifying the later more complicated simulations.

First was the task of deciding how detailed of a model was necessary to capture the effect of the helmet design while still maintaining a manageable model with respect to reconfiguration and solve time. The larger the model chosen, the more elements required to fill the model, and the longer the solve time. Additionally, if a more complicated model was chosen to represent the design, the mesh would inevitably have to be finer to capture the smaller details. This would, in turn, further lengthen the computation time. While a more complicated model would likely yield results slightly more similar to real life, a much simpler model is all that is needed to verify this design against a control model of a similar complexity.

The model was thus chosen to be as simple as possible. The shell portion of the helmet was modeled as a partial spherical shell of thickness 0.15 " and diameter 8.2 ". This thickness value and diameter were taken directly from an existing football helmet. The headform to simulate the head of a player was modeled as a sphere with a diameter of 6.2". [16] The subsequent internal padding within the helmet was modeled as a 1.0" thick 
spherical shell to fit within the helmet shell. A depiction of these components can be found in Figure 13 below.

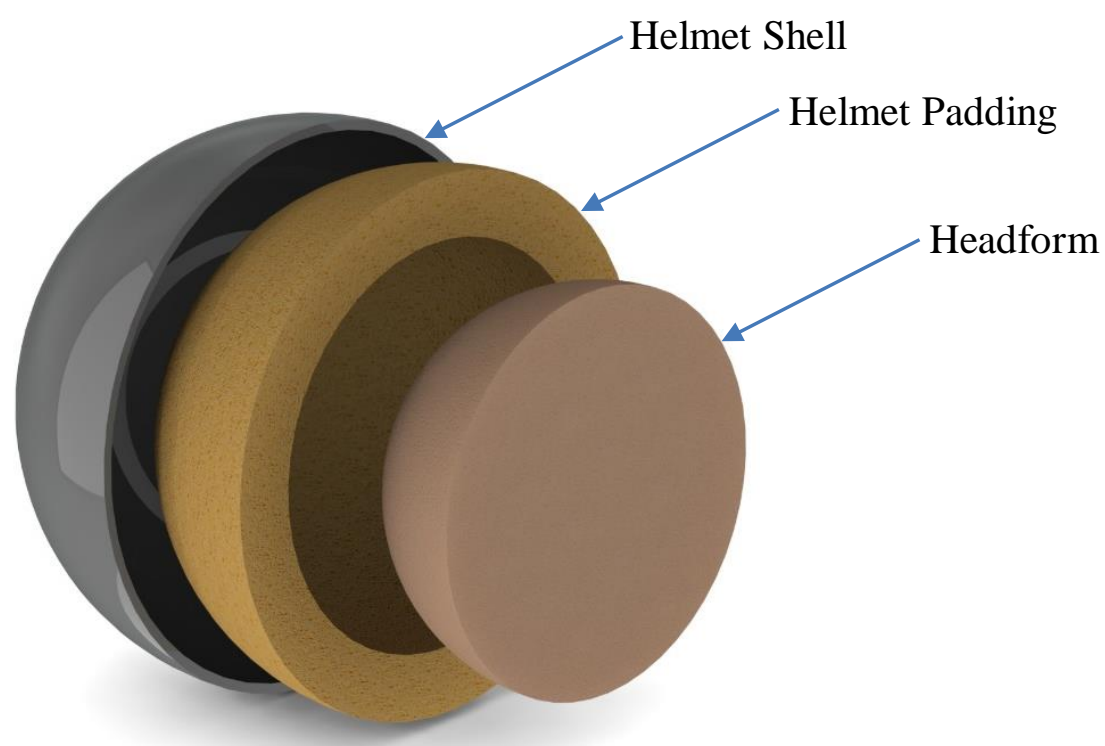

Figure 13. The simple spherical shell model used as the basis for the computer analysis.

Due to the symmetrical nature of the proposed model, it was possible to simplify the simulation even further. By modeling only half of the helmet assembly and applying boundary conditions to mimic the other half of the model, it was possible to achieve the same results as a full model but in half the time of running a full simulation. This concept can be seen in Figure 14. A technical drawing of this configuration can be found in APPENDICES

Appendix A. It's important to note here that even though only one quarter of the headform is being shown, it has been given properties that assign it the mass of half a head to maintain its relation to a half-model. 


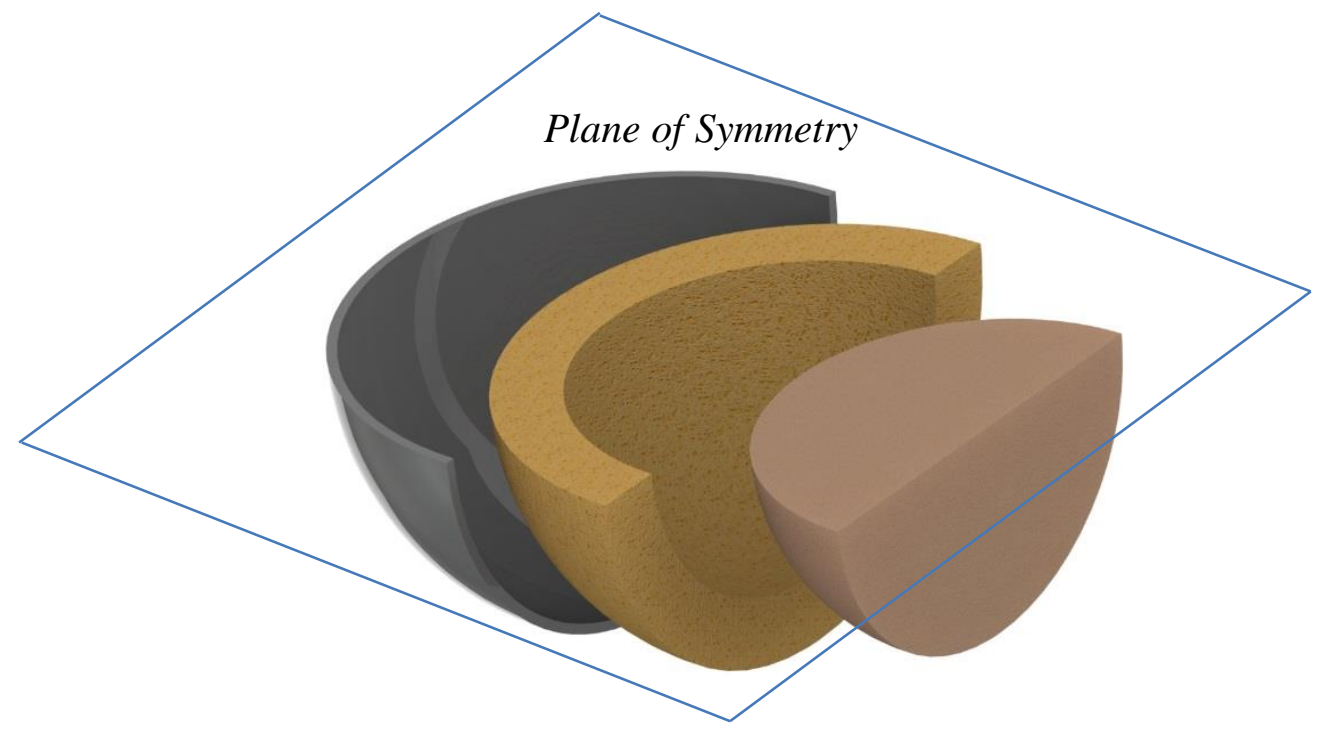

Figure 14. Half model used in the Abaqus simulations.

The solid models of each component were drawn in Solidworks 2015-2016. [17] The parts were then combined into an assembly in order to orient them correctly with respect to one another. Then each part was saved individually from the Solidworks assembly as an International Graphics Exchange Standard (IGES) file type by hiding and suppressing the other parts. This process ensured that the IGES geometries were all relative to a single set of global coordinate axes. Each part was then imported into Abaqus CAE, the finite element pre-processor and post-processor used for this analysis. Having all the parts preoriented saved a significant amount of time since the part orientation and mating tools in Abaqus are not as powerful as in Solidworks.

Each part was then given a section property that described its material characteristics: polycarbonate for the shell and low density foam for the padding, sub-padding and cores. The parts were then all meshed to generate the elements necessary to solve an FEA problem. The mesh characteristics for each part can be seen in Table 1. All the parts were 
brought into an assembly and, having been oriented properly ahead of time, required little additional manipulation. A model representing a rigid impact pad was also brought into the assembly.

Table 1. Element type and seed size for all the components of the Abaqus models.

\begin{tabular}{|c|c|c|c|}
\hline Part & Element Type & Seed size & $\begin{array}{c}\text { Approximate number of } \\
\text { elements }\end{array}$ \\
\hline Impact Surface & $\begin{array}{c}\text { Shell, Explicit, Linear, } \\
\text { Reduced Integration } \\
\text { (S4R) }\end{array}$ & 0.25 & 6,400 \\
\hline Helmet Shell & $\begin{array}{c}\text { 3D Stress, Explicit, } \\
\text { Linear (C3D8) }\end{array}$ & 0.075 & 40,000 \\
\hline Sub-padding & $\begin{array}{c}\text { 3D Stress, Explicit, } \\
\text { Linear (C3D8) }\end{array}$ & 0.25 & 1,000 \\
\hline Cores & $\begin{array}{c}\text { 3D Stress, Explicit, } \\
\text { Linear (C3D8) }\end{array}$ & 0.125 & 5,500 \\
\hline Helmet Padding & $\begin{array}{c}\text { 3D Stress, Explicit, } \\
\text { Linear (C3D8) }\end{array}$ & 0.20 & 3,200 \\
\hline Headform & $\begin{array}{c}\text { 3D Stress, Explicit, } \\
\text { Linear (C3D8) }\end{array}$ & 0.25 & \\
\hline
\end{tabular}

Establishing the proper contact regimes was the next step. Initially every contact situation, head to inner padding, outer padding to inner sub-padding, outer sub-padding to inner shell, cores to channels, and outer shell to impact surface, was given hard contact that allowed separation. This meant that none of the parts were permanently attached and they could all move and separate relative to each other. This presented problems because the simulation was predicting a large amount of relative movement and the parts were generating errors due to extremely rapid movement and deformation. After considering how each of the parts was going to be attached on a physical helmet, however, most of these contact interactions were converted to tie constraints in which all the DOF at the interface are equivalent. These constraints would not allow the parts to separate but, 
rather, they must remain in contact for the duration of the simulation. The only interactions that remained having separable contact were the headform with the padding and the shell with the impact surface. These two interfaces required this form of contact because they act this way in the real world. The other contacts represented boundaries where the parts would be velcroed or glued, which would eliminate any lateral movement. The contact method used for each interface is shown in Figure 15.

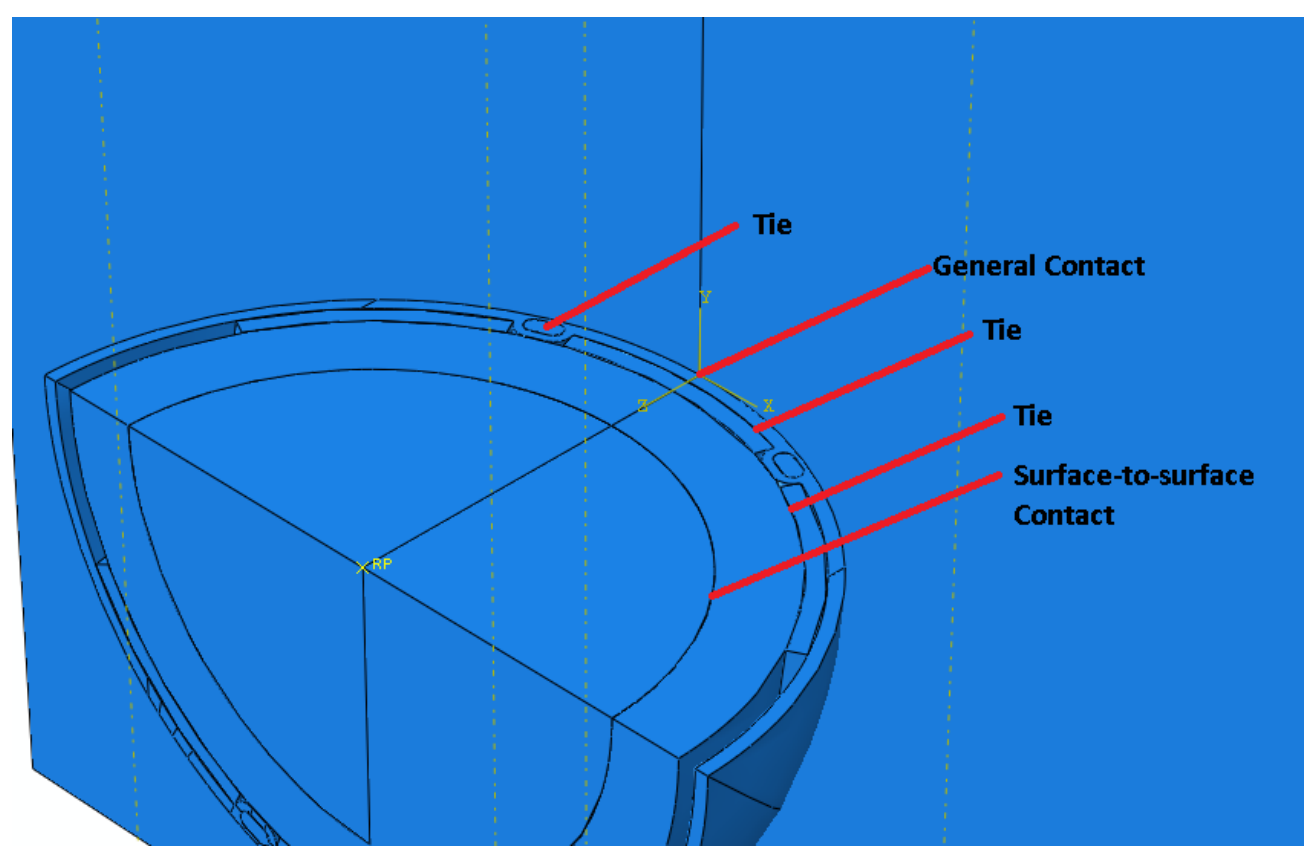

Figure 15. Denotation of the contact method used at each interaction area.

The helmet parts were then given an initial velocity applied in the step before the analysis was to take place. This ensured that the helmet would have no acceleration at the start of the analysis, but rather only an initial velocity. The analysis step was designed to be a dynamic explicit step to be run over the duration of 0.010 seconds, or 10 milliseconds. After running the model several times it was determined that the model had to be run for a full 15 milliseconds to capture the full acceleration spike from the impact. The acceleration data for the entire impact is required to calculate the Severity Index of an 
impact. A full accounting of the properties used in the simulations can be seen in Appendix B: Properties used in Abaqus/CAE simulations.

To gather the necessary acceleration data from the headform, a history output request was required. Abaqus generates many of its outputs based on what the user requests from its post processing suite. This is done simply by asking Abaqus to monitor the acceleration of a point on the head-form so that the data could then be output. The acceleration data was output at a frequency of $133,000 \mathrm{~Hz}$ which is a faster capture rate than the NOSCAE specification.

\subsection{Model Validation}

One of the most important things to remember when analyzing FEA results is that they are meaningless without having been validated. The simulations need to be compared to real-world data to ensure that the computer is correctly solving the loading case. For this model the validation was done in a number of steps.

\subsubsection{Helmet Shell}

The first component to validate was the shell of the helmet. The goal of this validation was to compare the stress and displacement values of a hemispherical shell under top loading (Figure 16) between an Abaqus simulation and physical equations. This would indicate if Abaqus was properly representing the shell of the helmet. The constitutive equations from Roarks's Formulas for Stress and Strain for a partial spherical shell with a load concentrated on a small circle at the pole with any edge support can be seen below in equations 7, 8 and 9. Factors $\mathrm{A}, \mathrm{B}$ and $\mathrm{C}$ are given by equation 10 and Table 2. [18] 


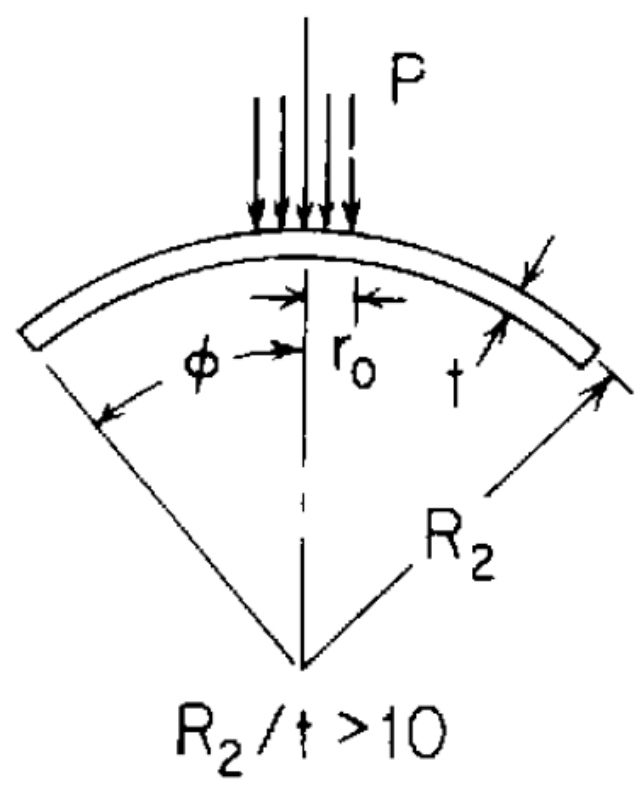

Figure 16. Image accompanying equations for a partial spherical shell from Roark's Formulas for Stress and Strain indicating the variables needed to complete the analysis. [18]

$$
\begin{gathered}
\text { Deflection }=\delta=-A \frac{P R_{2} \sqrt{1-v^{2}}}{E t^{2}} \\
\text { Max membrane stress }=\sigma_{1}=\sigma_{2}=-B \frac{P \sqrt{1-v^{2}}}{t^{2}} \\
\text { Max bending stress }={\sigma^{\prime}}_{1}={\sigma^{\prime}}_{2}=-C \frac{P(1+v)}{t^{2}} \\
\mu=r^{\prime}{ }_{o}\left[\frac{12\left(1-v^{2}\right)}{R_{2}{ }^{2} t^{2}}\right]^{1 / 4}
\end{gathered}
$$

Table 2. Table of $A, B$ and $C$ values with respect to values of mu to calculate maximum deflection, membrane stress, and bending stress for a partial spherical shell under loading at one pole. [18] 


\begin{tabular}{l|ccccccccc}
$\mu$ & 0 & 0.1 & 0.2 & 0.4 & 0.6 & 0.8 & 1.0 & 1.2 & 1.4 \\
\hline$A$ & 0.433 & 0.431 & 0.425 & 0.408 & 0.386 & 0.362 & 0.337 & 0.311 & 0.286 \\
$B$ & 0.217 & 0.215 & 0.212 & 0.204 & 0.193 & 0.181 & 0.168 & 0.155 & 0.143 \\
$C$ & $\infty$ & 1.394 & 1.064 & 0.739 & 0.554 & 0.429 & 0.337 & 0.266 & 0.211
\end{tabular}

The Abaqus model representing this loading can be seen in Figure 17. The maximum displacement observed in the simulation was 5.8\% larger than the formulas indicated and the max stress was $0.68 \%$ greater in the simulation than the formulas predicted. This low percentage error for both the displacement and stress prove that the simulation is accurately representing this shell correlation. The Matlab code for solving the Roark equations is shown in Appendix C: Max Bending and Spherical Stress Matlab Code

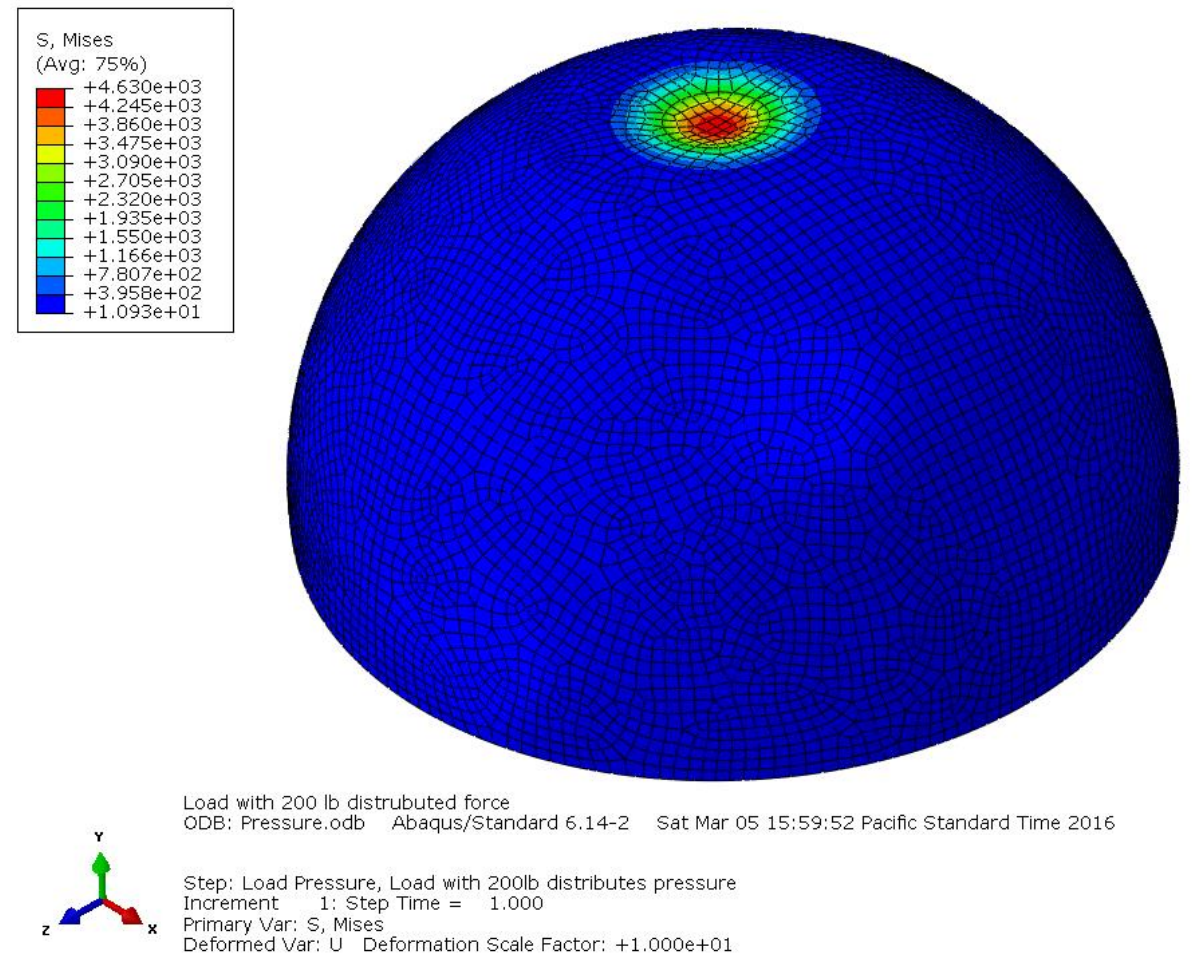

Figure 17. Spherical shell validation model.

In addition to the displacement and stress analysis on the hemispherical shell, a convergence study was run to determine the necessary seed size for the shell. The 
convergence plot for displacement and stress can be seen in Figure 18 and Figure 19 respectively. From this analysis a seed size of 0.125 was determined to be adequate for the shell. A seed of .075 was actually used in the simulations in order to have the correct density of elements through the thickness of the shell. Three elements were necessary since the explicit elements used employed linear shape functions. At least three elements were needed to allow for wave propagation through the shell.

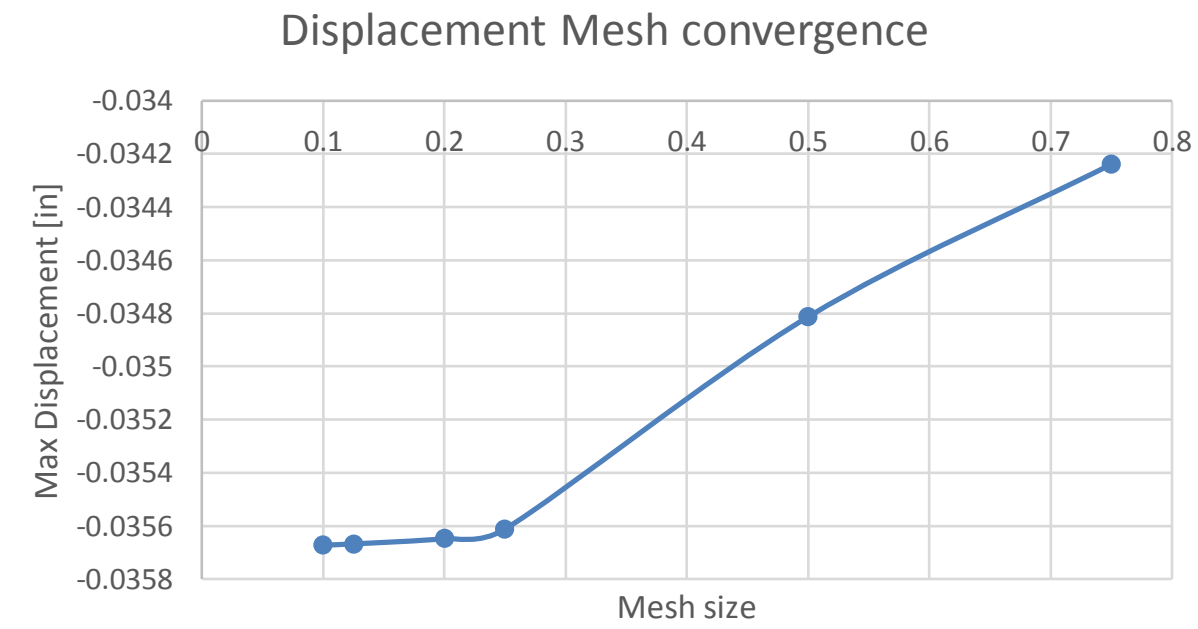

Figure 18. Mesh convergence plot for max displacement from the spherical shell analysis. 


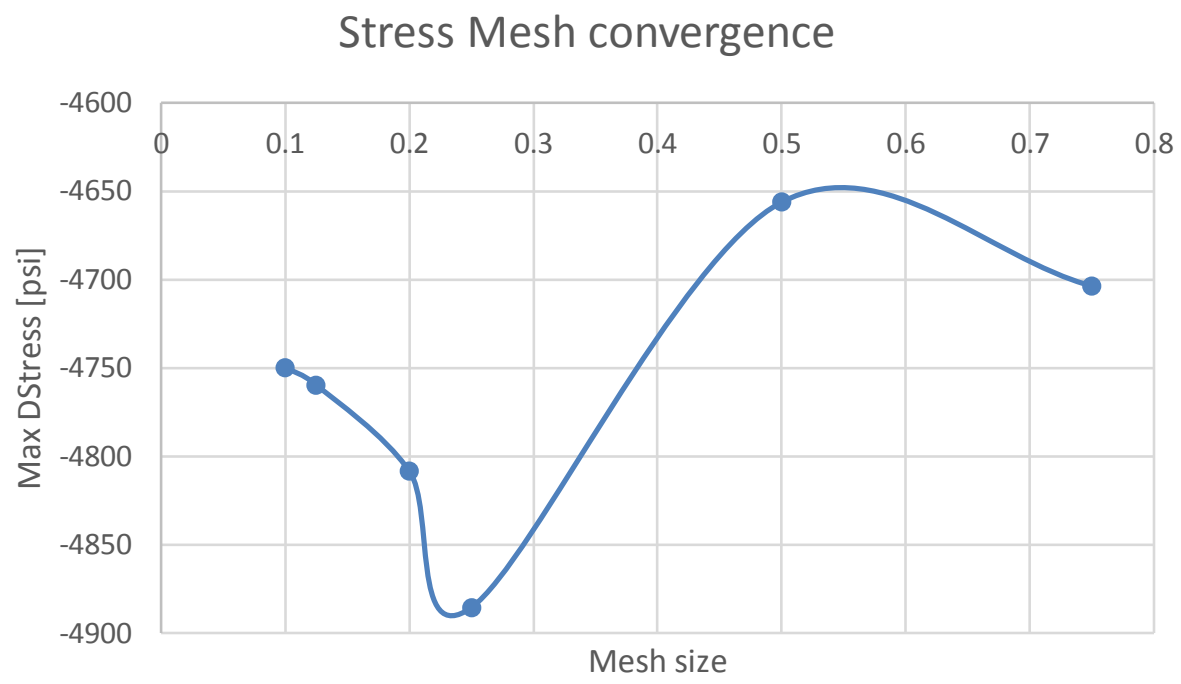

Figure 19. Mesh convergence plot for stress from the spherical shell analysis.

\subsubsection{Helmet Padding}

The next modeling challenge to overcome was how to accurately model the football helmet padding. All the physical helmets available for examination used a type of padding called Schutt Air XP padding. (Figure 20) This state of the art football padding made out of thermoplastic polyurethane is formed into a honeycomb-like structure which is excellent at absorbing impact energy. Additionally, this was the padding available to be used in the small scale testing, so it was important to try modeling this first. 


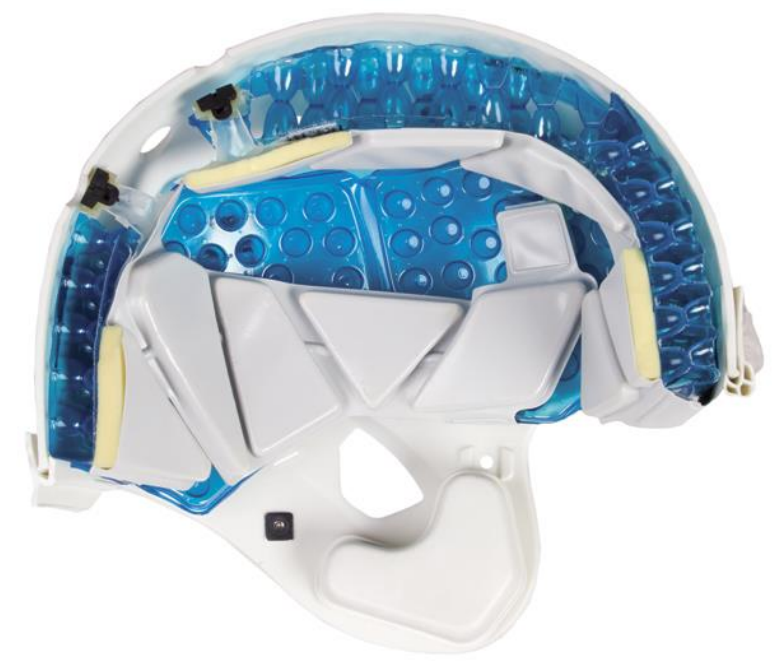

Figure 20. Schutt Air XP padding. [19]

Because the padding in the simulation model is a solid foam (not a honeycomb truss) and trying to model and run a finite element simulation with honeycomb structure padding in the full model would be needlessly complicated and time consuming, it was necessary to identify effective elastic properties for the Air XP padding. This material property could then be applied to the solid padding in the full simulation in the form of stress and strain values. To achieve these results, a sample of the Air XP padding was modeled in Solidworks and imported to Abaqus CAE. The model was reduced to surfaces and each surface was given a wall thickness of 0.035 " as well as the material properties of thermoplastic formed polyurethane. [20] (Appendix D: Thermoplastic Polyurethane Material Properties) The top of the padding was then made rigid and induced to move downward at a set rate. The simulation captured the stress exhibited by the deforming plastic on the rigid top as well as the total deflection for every moment in time. The deflection values could easily be converted into strain values and paired with their 
respective stress values to give a stress-strain relationship for the Air XP padding as a whole. The Abaqus simulation is shown in Figure 21.

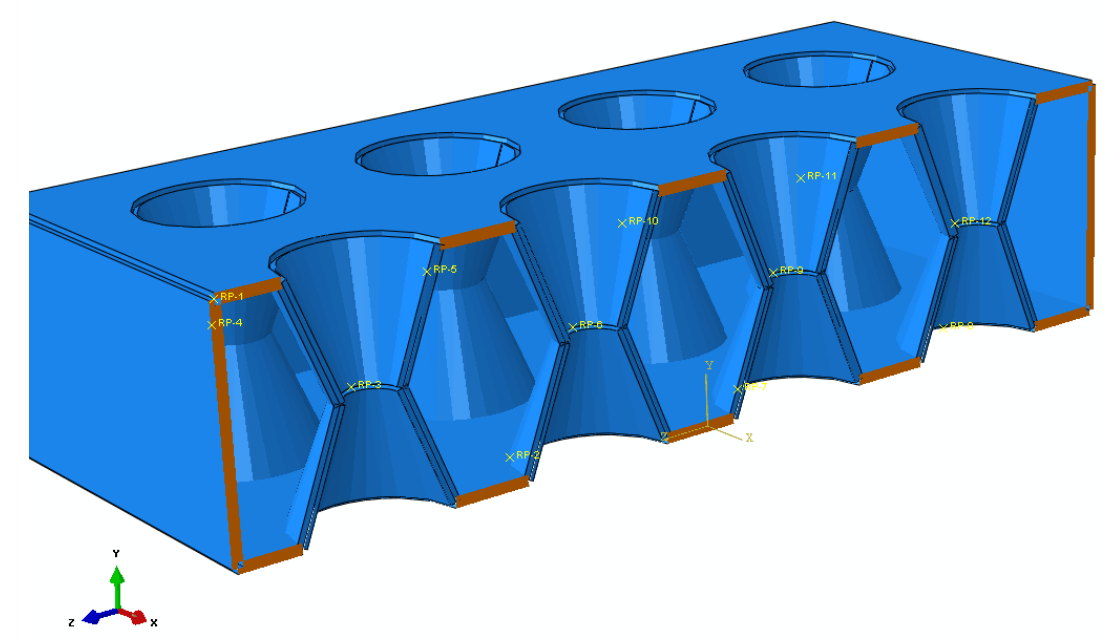

Figure 21. Abaqus model of thermoplastic polyurethane Schutt Air XP padding. Shown as a section view to display internal features.

Unfortunately, the simulation is highly unstable and would yield no results unless the sides of the padding were constrained in every direction except the compressive direction. These results ended up not matching the small scale testing and were, therefore, rendered useless.

The next attempt at simulating padding came from a Ford Motor Company handbook on impact reducing foams in bumper applications. A low density polyurethane foam with density of $88 \mathrm{~g} / \mathrm{l}$ from the handbook was used to represent the helmet padding. The handbook reported stress and strain data at 70 degrees Fahrenheit, based on impact tests done at a Ford facility. [21] The stress-strain plots used for the simulation as well as a 
table representation of the data can be found in Appendix E: Low Density Foam Material Properties.

The testing procedure called out for a $60 \times 60$ millimeter block to be impacted from above at a rate of $5 \mathrm{mph}$. By modeling an analogous experiment, the proper material model for the foam was able to be determined through trial and error. The stress and strain data for the foam was input into Abaqus and applied to the foam block. Once the simulation was run, the resulting stress and displacement data could be plotted against the original data from the handbook to check for compliance. It was ultimately determined that Abaqus's "Low Density Foam" material model was the best suited to the application with Hyperfoam and Hyperelastic material models being among those tested. The handbook data and Abaqus low density foam data plotted against each other can be seen in Figure 22 and the Abaqus model used of the analysis in Figure 23. It should be noted here that this data could only be achieved by restricting the sides of the block from moving outward. The gives the foam an effective Poisson's ratio of zero. This should have been the case regardless since low density foams have a default Poisson's ration of zero in Abaqus. The simulation was exhibiting unusual behavior, however, and the only solution was to force the zero Poisson's ratio condition. This suggests that the padding will have to be artificially constrained in the full model to accurately represent the foam. Mesh convergence was also carried out for the foam block. A mesh convergence plot for the foam can be found in Figure 24. It can be clearly seen that the seed size of 0.25 and 0.1 are almost identical. A final seed size of 0.25 was chosen for the foam components of the future models. 


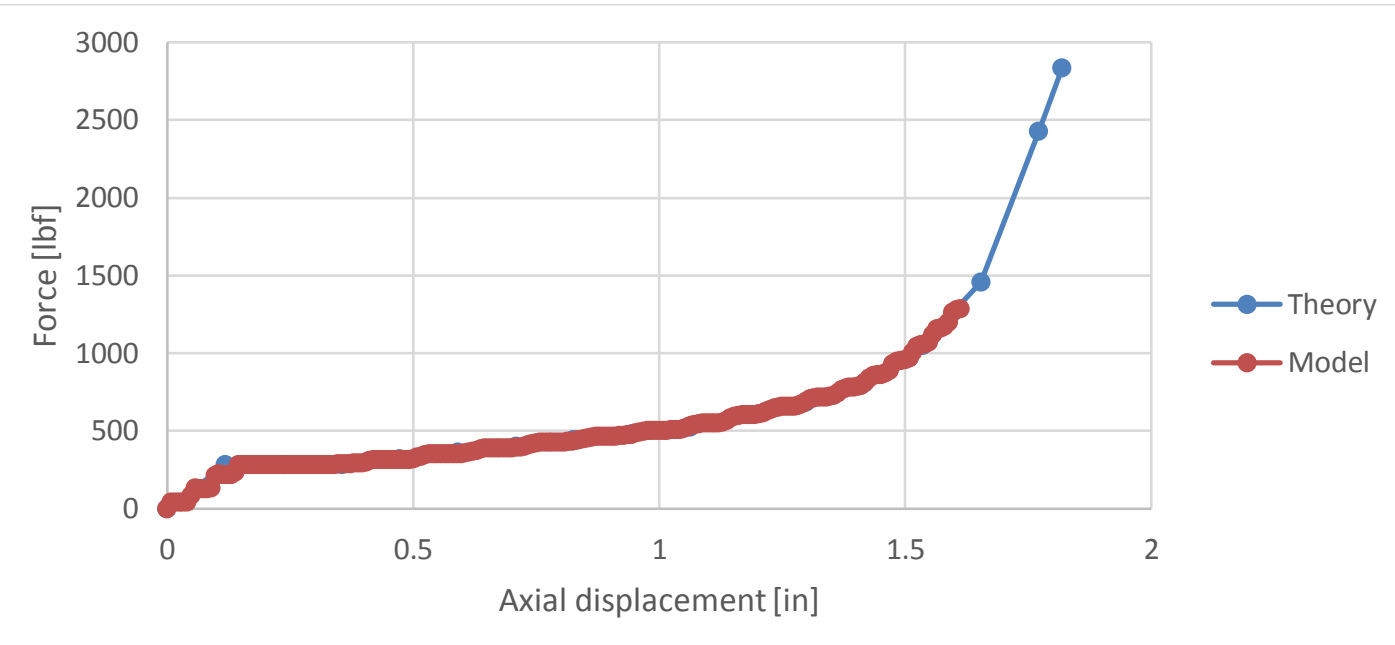

Figure 22. Force and displacement data from the Ford handbook (theory) plotted with data from an analogous Abaqus simulation (model).

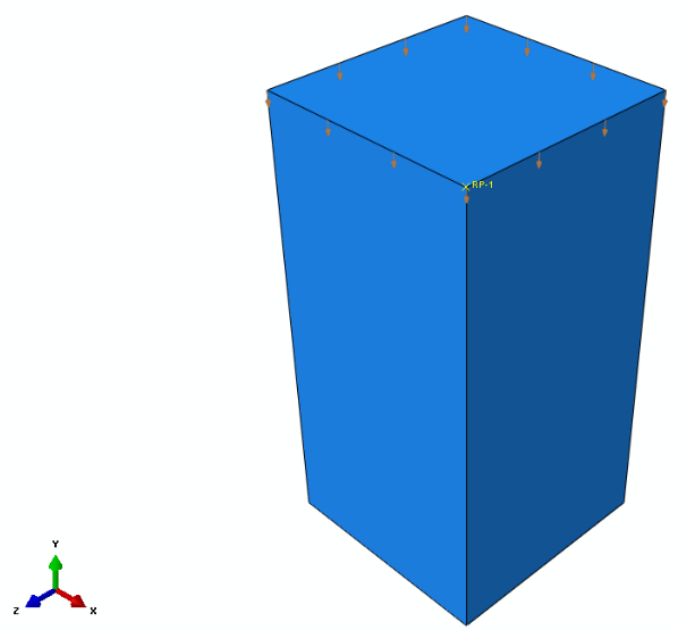

Figure 23. Foam block test model used to verify that Abaqus was accurately representing the data from the Ford handbook. 


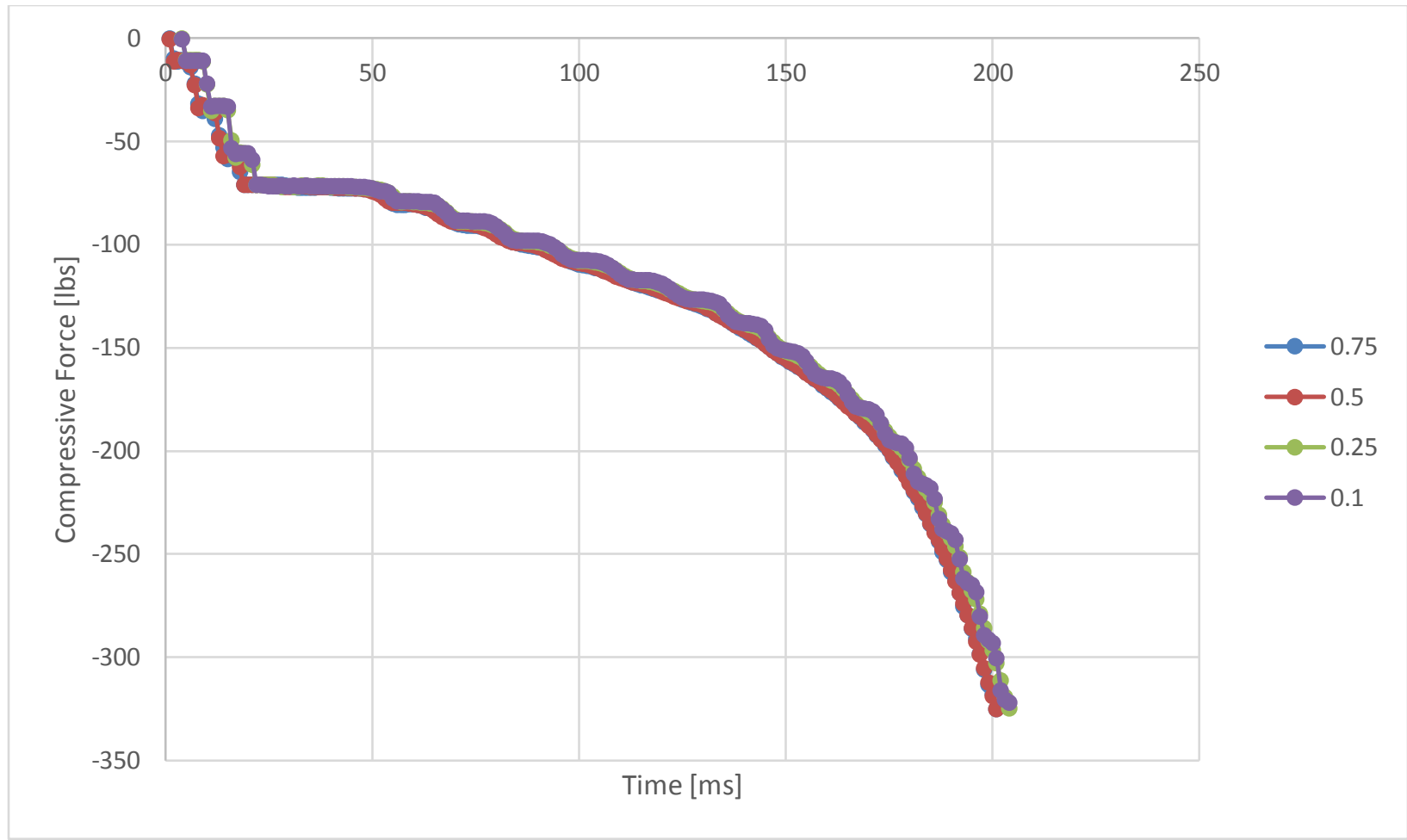

Figure 24. Compressive force over time for different seed values for the foam validation test. The 0.25 and 0.1 seed size were almost identical so a final seed size of 0.25 was chosen.

Now that it had established that the foam was being properly modeled, it had to be compared to the real life helmet padding to check for similarity. To do this, data from a real life helmet impact was needed to compare to a simulated impact. These data were generated from the testing of a 3-D printed model of the impact diffusion helmet design and a control model. The two prototypes were impacted from a set height and an accelerometer captured the acceleration of the "head". This small-scale testing was carried out by Michael Schuster. [22] Figure 25 shows the configuration of the components of the test. Once this data had been collected, it was only a matter of postprocessing it to determine the maximum acceleration of each impact. 


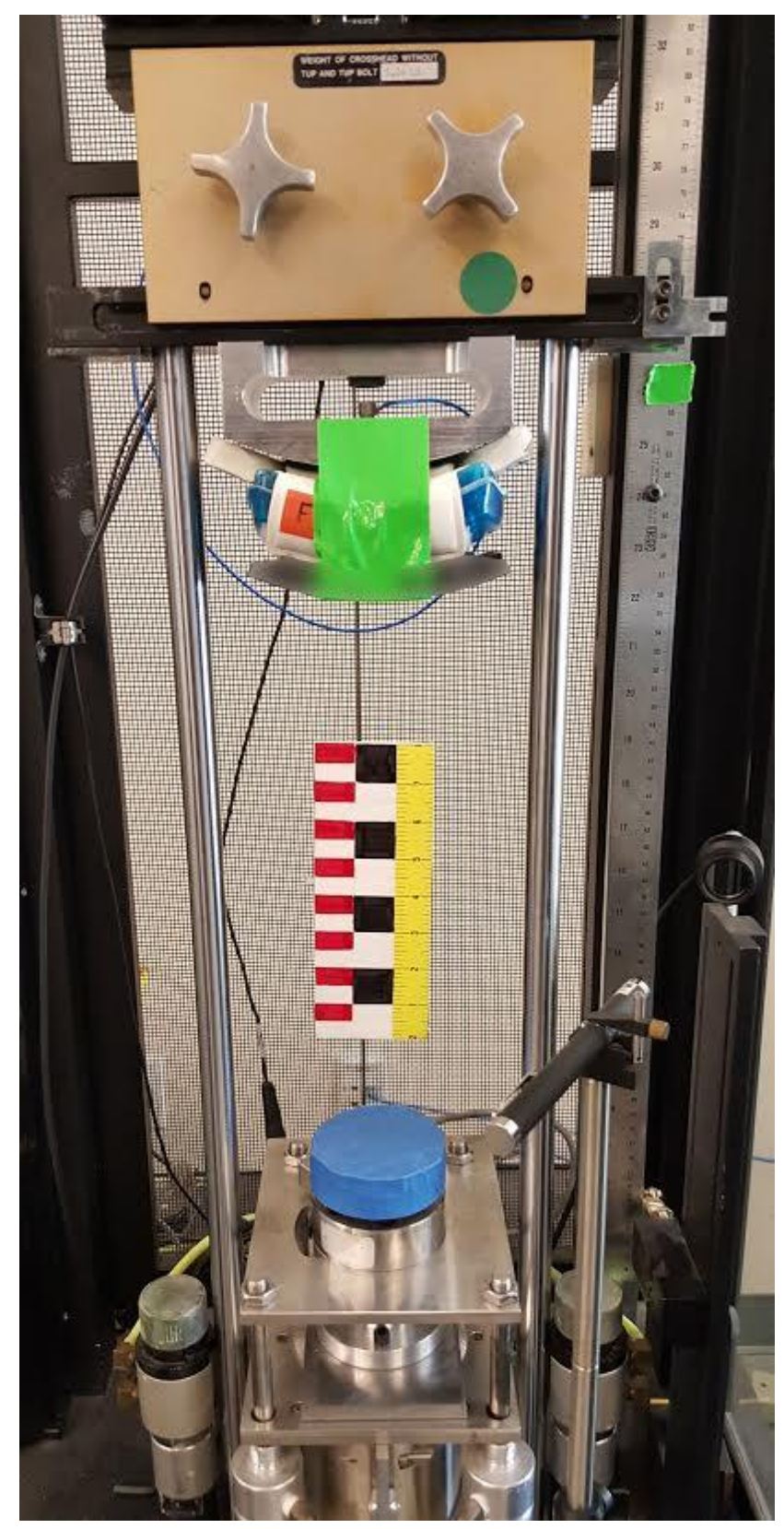

Figure 25. Small scale testing configuration and apparatus. The 3-D printed prototype was taped to the aluminum impactor beneath Schutt Air XP padding. This assembly was dropped onto the impact surface (blue) and the acceleration of the impactor was recorded. [22]

Now that the testing had been completed, a simulation identical to the test was developed to verify the padding properties. Both a control simulation and a channeled simulation were made to verify peak acceleration results from the control prototype as well as the 
results from impact diffusion design. Those models are shown in Figure 26 and Figure 27. The models were initially tested with foam material property data gathered from simulating the Shutt Air XP padding, but the results were orders of magnitude from the test data. At this point the data from the Ford foam handbook were substituted into the simulations. Unfortunately, the data from these tests showed a slightly higher peak acceleration than the data from the physical tests. In order to match the peak accelerations from each impact test, the stress-strain data of the padding was altered. At each strain level, the stress value was divided by four. This simple change led to the maximum acceleration in the control test being $5.9 \%$ different than the physical test and the channeled model being $15.4 \%$ different. These difference were determined to be adequate for the type of analysis that was to be performed. Since all subsequent models were going to be compared to a control helmet, the padding precisely mimicking real-life padding wasn't a priority as long as all the models used the same material properties. Figure 28 and Figure 29 depict the acceleration pulse from the control simulation and the physical testing respectively. 


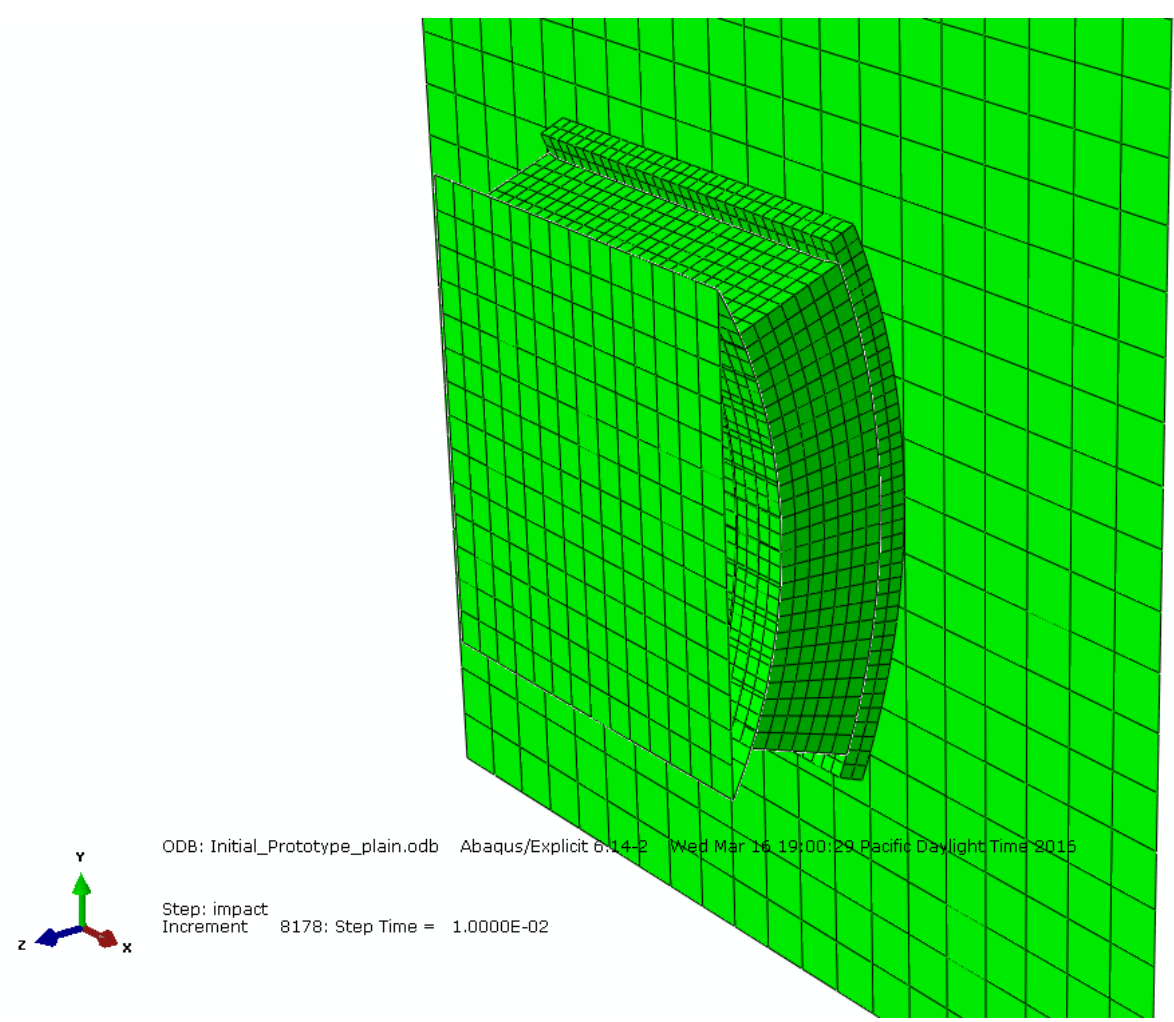

Figure 26. Control model used to validate padding properties.

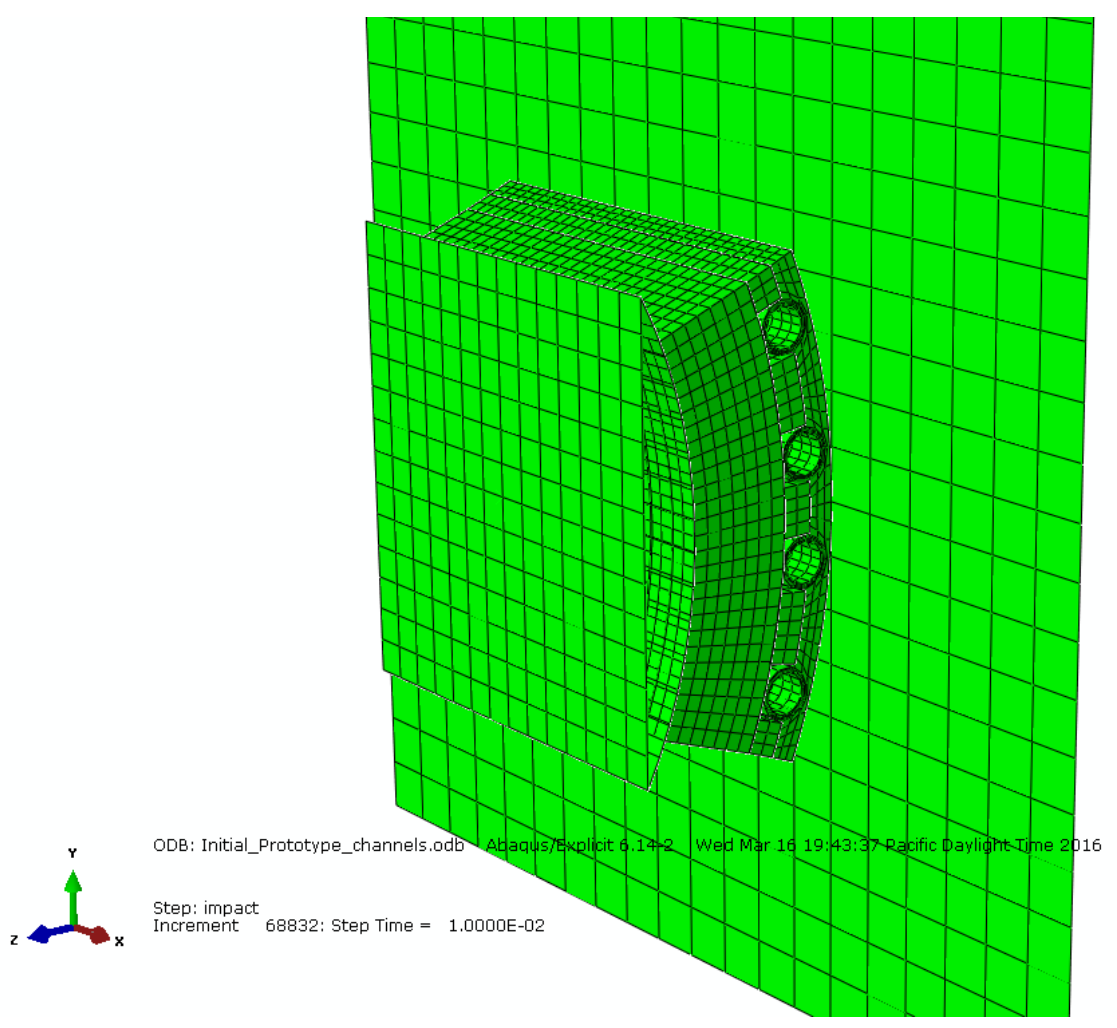

Figure 27. Channeled model used in validating padding properties. 


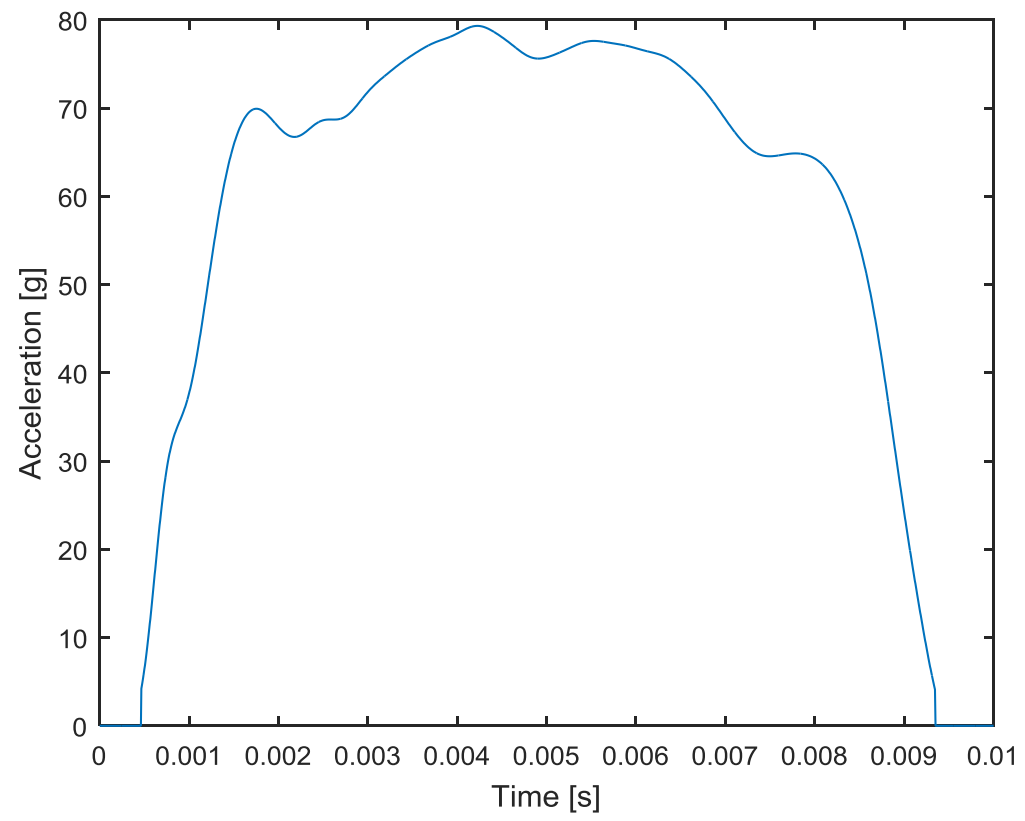

Figure 28. Acceleration pulse from the control prototype simulation.

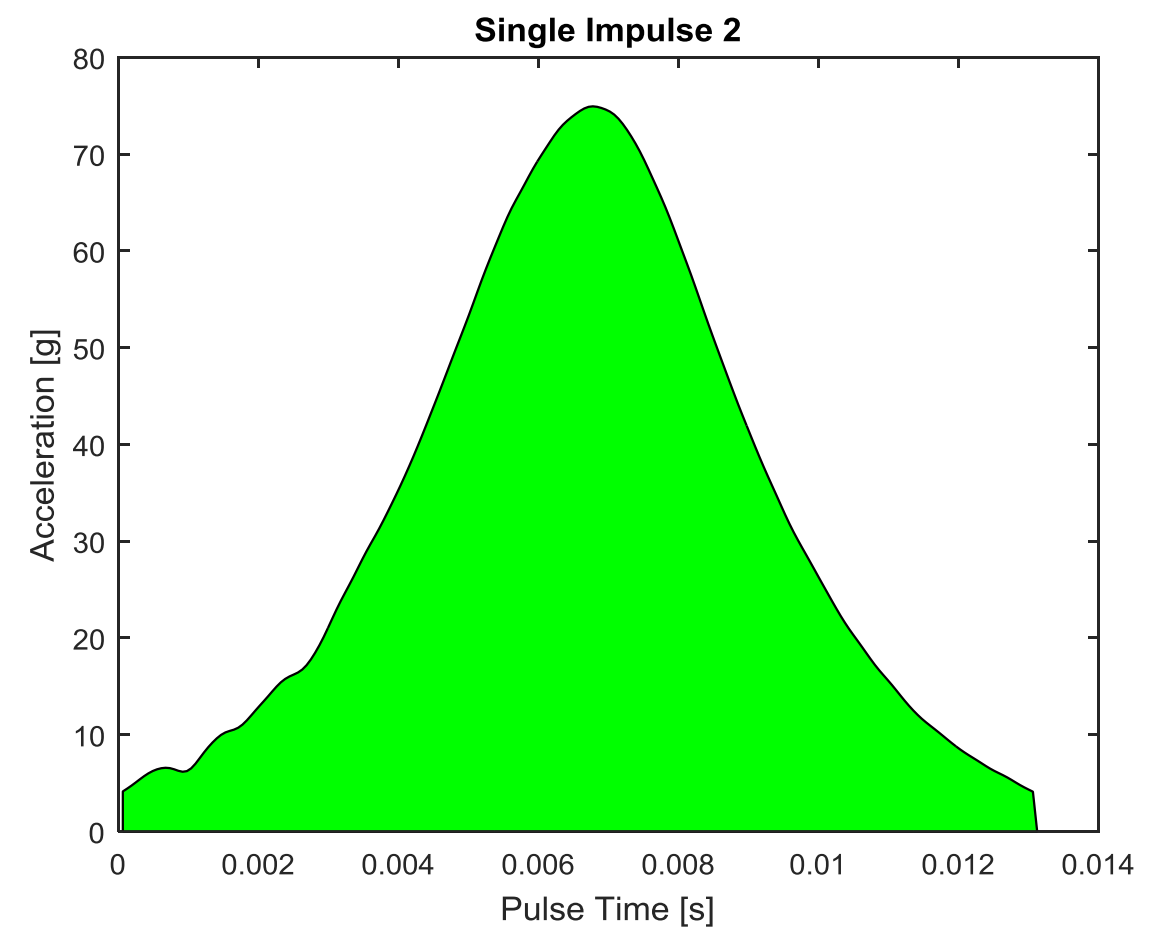

Figure 29. Acceleration pulse from the control physical testing impact. 


\section{Comparative Analysis}

Now that the models had been fully developed and validated, it was time to begin the analytical phase of the project. The objective was to determine if the channeled geometry provided significant impact reduction properties. Even a Severity Index reduction of as little as 5\% would be considered a success since even this little of a reduction would aid in reducing the effect of repeated impacts over time. Initially, an experiment was run to determine which controllable variables had the largest effect on the impact reduction of the design. Then a follow-up study was performed that optimized the variables that had the largest effect of reducing the impact energy. Lastly, a number of final verification simulations were run to ensure that the optimized design was the best possible configuration. A representative Abaqus model for the analysis that was performed can be seen in Figure 30 below.

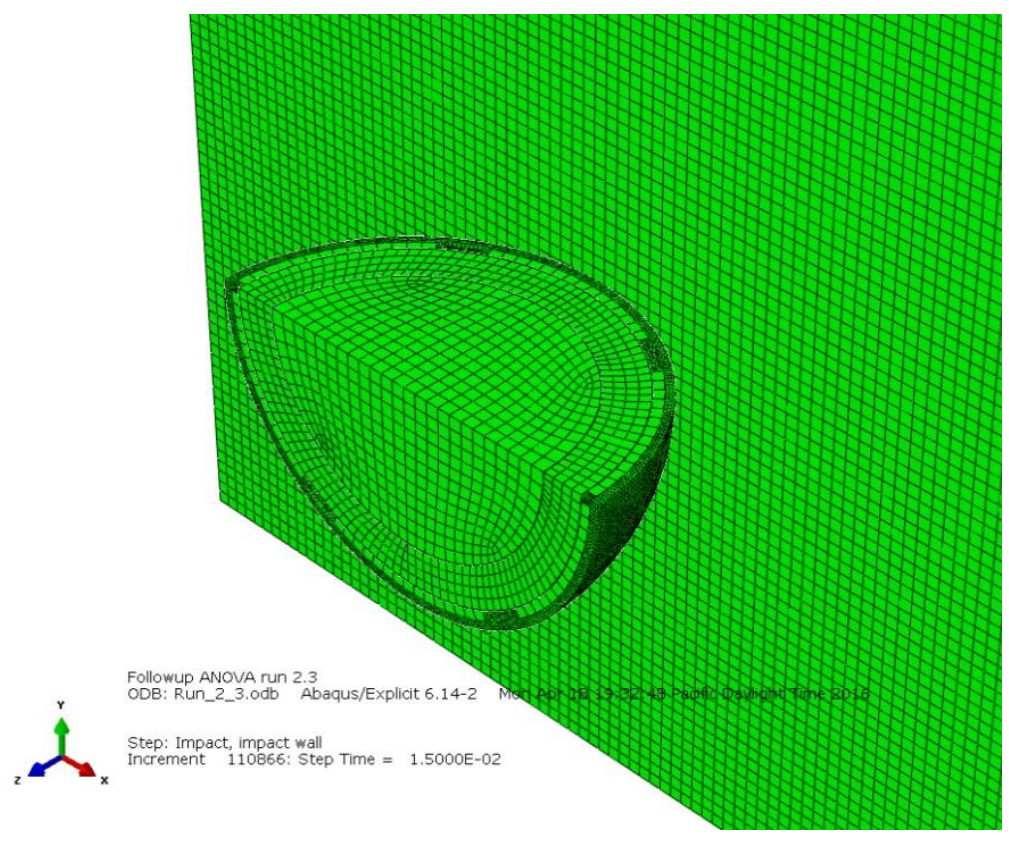

Figure 30. Representative Abaqus model for the analysis performed. The changes between the runs of the analysis involved changing the geometry of the helmet shell. 


\subsection{Statistical Modeling}

Since there were no definite geometries or dimensions, the variable aspect of this design had to be determined experimentally. Unfortunately, it would be extremely time consuming to test every configuration of the design. For example, if there were four design variables to test and each variable had four levels, it would require $4^{4}$, or 256 , experimental runs to capture every possible configuration. This is where the concept of design of experiments (DOE) is applicable. DOE can be used for everything from choosing the best between two alternatives to selecting the key factors that affect a response. [23] The latter application will be the one employed for this analysis.

\subsection{Screening Analysis}

\subsubsection{Background}

The first task for any design optimization is to screen out the variables that have no effect on the desired outcome. [23] This allows the analyst to then focus on the important factors as opposed to wasting time and money running experiments with variables that have little to no effect on the studied result.

In this project, the design of the impact reduction helmet was still very fluid prior to analysis. Many factors of the helmet were able to be changed and modified and still conform to the overarching design. The factors that were variable in this analysis are listed below in Table 3 . 
Table 3. List of factors available to be modified in the design of the impact reduction helmet.

\begin{tabular}{|l|r|}
\hline \multicolumn{2}{|c|}{ Factor } \\
\hline $\mathbf{1}$ & Minimum Shell Thickness \\
\hline $\mathbf{2}$ & Channel Shape \\
\hline $\mathbf{3}$ & Channel Diameter \\
\hline $\mathbf{4}$ & Number of Channels \\
\hline $\mathbf{5}$ & Core Material \\
\hline $\mathbf{6}$ & Impact Velocity \\
\hline
\end{tabular}

To determine which variables were statistically significant and which had little effect on the impact reduction properties of the helmet, an approach of DOE called analysis of variation (ANOVA) was used. [23,24] The screening ANOVA study was determined to have six factors at two levels each. The levels would then be paired by applying a fractional factorial design, an ANOVA approach. This procedure reduces the total number of experiments to be run by cleverly matching factors in a way that can later be analyzed and subjected to statistical breakdown. The type of design used here is called a $2_{\mathrm{III}}^{6-3}$ fractional factorial design. The two refers to the number of levels of each factor, the six to the number of factors being tested, the three to the class of the fractional factorial design, and the III to the resolution of the analysis. The resolution identifies the amount that the main effects are aliased, or confounded. This should be avoided in a typical analysis, but resolution III designs are commonly used for screening analyses since they allow a larger number of factors to be tested in fewer runs. This fractional factorial design reduced the number of runs in the experiment from $2^{6}$ to $2^{6-3}$, or from 64 runs to 8 runs. The screening ANOVA analysis table can be seen in Table 4 and the run table is shown in Table 5. The run table works by combining the levels for each factor for every run. For example, Run 1 would consist of a minimum shell thickness of .05”, a circular channel 
shape, a channel diameter of .20", 4 channels, foam cores in the channels, and an impact speed of $100 \mathrm{in} / \mathrm{s}$. A representative drawing of the screening analysis model can be found in Appendix F: Drawing of Representative Experimental Model. The levels of this screening ANOVA were chosen with the helmet geometry limitations in mind. The minimum shell thickness was chosen to prevent the shell from getting to thin while still allowing the channels to be slightly imbedded in the shell. The diameters were selected because .20 " and .30 " approximately represented the channel sizes indicated by the sponsor. The number of channels was decided arbitrarily. The initial velocities were chosen because they closely emulate the velocities seen in the small scale testing.

Table 4. Screening ANOVA factors and levels.

\begin{tabular}{|c|c|c|c|}
\hline & & \multicolumn{2}{|c|}{ Levels } \\
\hline \# & Factor & -1 & +1 \\
\hline 1 & Minimum Shell Thickness & .05 & $.10 "$ \\
\hline 2 & Channel Shape & Circle & Oval \\
\hline 3 & Channel Diameter & $.20 ”$ & $.30 "$ \\
\hline 4 & Number of Channels & 2 & 4 \\
\hline 5 & Material in Cores & Nothing & Foam* \\
\hline 6 & Initial Velocity of Helmet & $80 \mathrm{in} / \mathrm{s}$ & $100 \mathrm{in} / \mathrm{s}$ \\
\hline
\end{tabular}

Table 5. Run table for a $2{ }_{I I I}^{6-3}$ Fractional Factorial ANOVA design.

\begin{tabular}{|c|c|c|c|c|c|c|}
\cline { 2 - 7 } \multicolumn{1}{c|}{} & \multicolumn{7}{c|}{ Factor/ Level } \\
\hline Run & $\mathbf{1}$ & $\mathbf{2}$ & $\mathbf{3}$ & $\mathbf{4}$ & $\mathbf{5}$ & $\mathbf{6}$ \\
\hline $\mathbf{1}$ & -1 & -1 & -1 & +1 & +1 & +1 \\
\hline $\mathbf{2}$ & +1 & -1 & -1 & -1 & -1 & +1 \\
\hline $\mathbf{3}$ & -1 & +1 & -1 & -1 & +1 & -1 \\
\hline $\mathbf{4}$ & +1 & +1 & -1 & +1 & -1 & -1 \\
\hline $\mathbf{5}$ & -1 & -1 & +1 & +1 & -1 & -1 \\
\hline $\mathbf{6}$ & +1 & -1 & +1 & -1 & +1 & -1 \\
\hline $\mathbf{7}$ & -1 & +1 & +1 & -1 & -1 & +1 \\
\hline $\mathbf{8}$ & +1 & +1 & +1 & +1 & +1 & +1 \\
\hline
\end{tabular}

It is important to note that all the properties of the simulations that are not included in the ANOVA test must remain constant for the results to have any meaning. The properties of 
the model that remained the same were the material properties of all the parts, the boundary conditions, the step properties, and the section of the helmet where channels were placed. In this case the channels were distributed through a 90 degree portion in the middle of the helmet. (Figure 31)

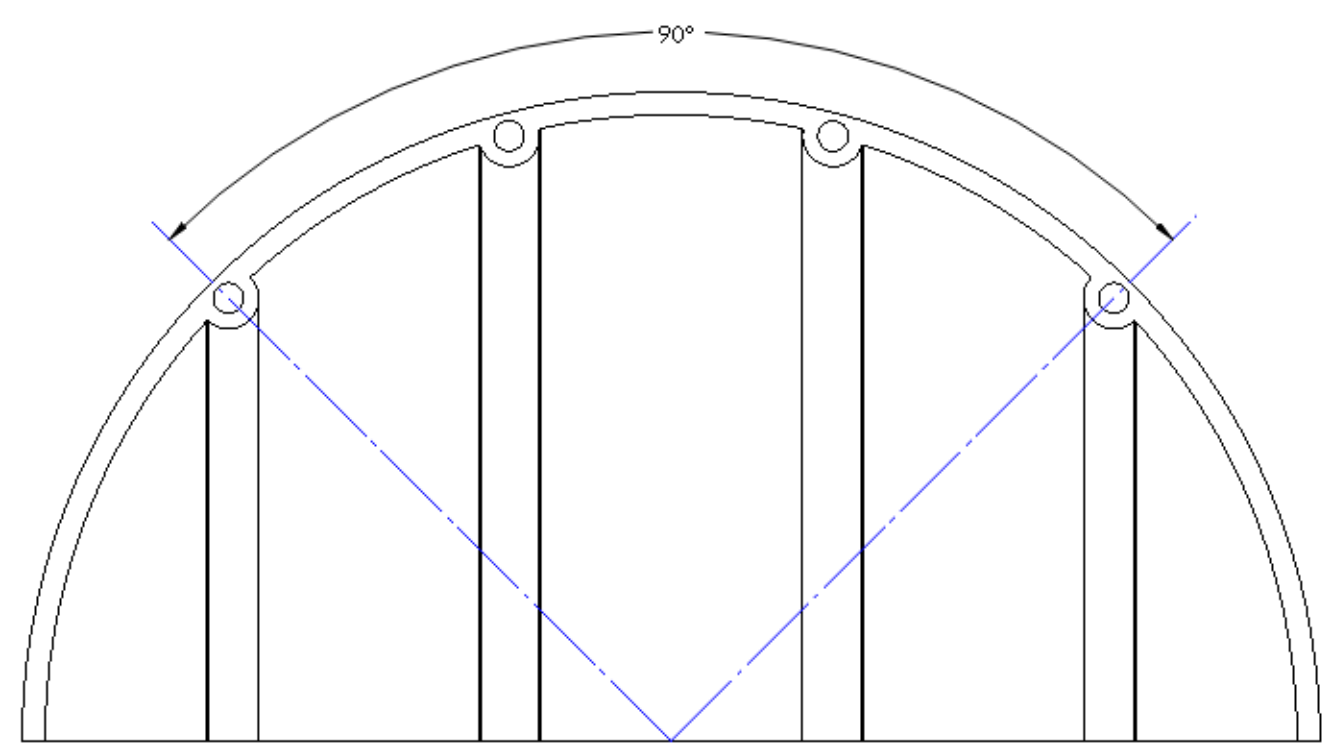

Figure 31. Channels spaces evenly in a 90 degree wedge.

\subsubsection{Results}

It was determined that the best criteria to judge the models was the Severity Index. This metric, which is what is used by NOCSAE to quantify a helmet's ability to absorb an impact, is what makes it the best judge of impact reduction. The Severity Index for each run was input into Minitab 17.2.1, a statistical analysis software, to determine the relationships between each of the factors and the Severity Index. [25] Table 6 shows the Severity Index values for each of the runs and Figure 32 shows the main effects plot generated by Minitab. This main effects plot graphically indicates how each of the factors contributes to the SI. 
Table 6. Calculated Severity Index for the screening ANOVA. The control severity indices are also included to allow for easy comparison between the experimental trials.

\begin{tabular}{|c|c|c|}
\hline Run & Severity Index & Impact Speed \\
\hline 1 & 124.34 & $100 \mathrm{in} / \mathrm{s}$ \\
\hline 2 & 115.23 & $100 \mathrm{in} / \mathrm{s}$ \\
\hline 3 & 71.28 & $80 \mathrm{in} / \mathrm{s}$ \\
\hline 4 & 74.47 & $80 \mathrm{in} / \mathrm{s}$ \\
\hline 5 & 74.46 & $80 \mathrm{in} / \mathrm{s}$ \\
\hline 6 & 76.16 & $80 \mathrm{in} / \mathrm{s}$ \\
\hline 7 & 117.86 & $100 \mathrm{in} / \mathrm{s}$ \\
\hline 8 & 123.82 & $100 \mathrm{in} / \mathrm{s}$ \\
\hline Control & 73.87 & $80 \mathrm{in} / \mathrm{s}$ \\
\hline Control & 117.52 & $100 \mathrm{in} / \mathrm{s}$ \\
\hline
\end{tabular}

\section{Main Effects Plot for SI}

Fitted Means

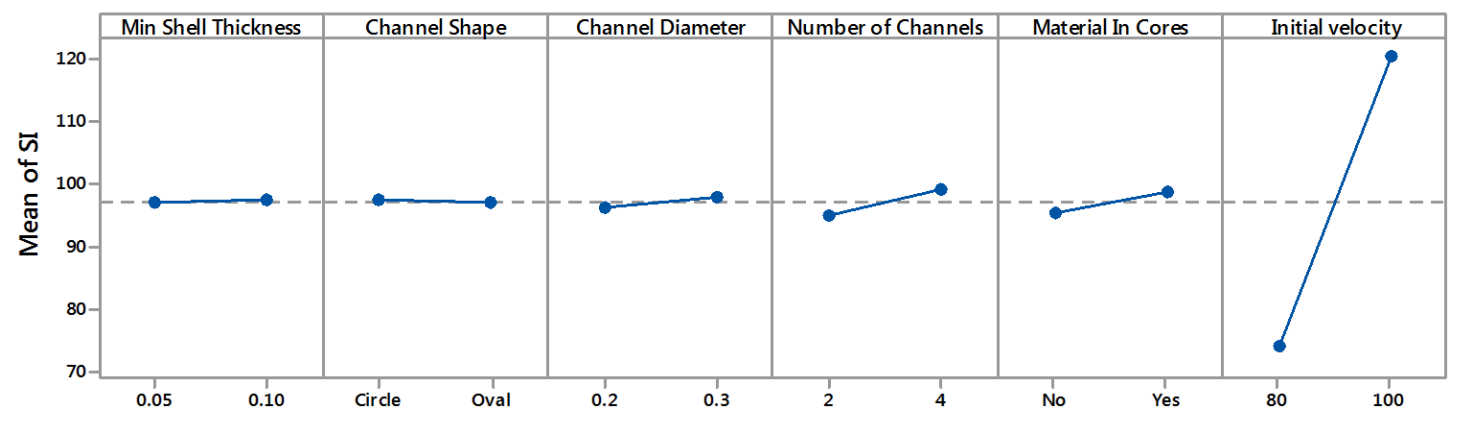

Figure 32. Main effects plot generated for the screening ANOVA.

The SI values themselves were calculated for each run from the acceleration data gathered from the simulation. The raw data captured at $133,333 \mathrm{~Hz}$ was first imported into a Matlab code for filtering. The raw and filtered data can be seen in Figure 33 and Figure 34 respectively for Run 1 . The data was filtered using a NOCSAE compliant four pole low pass Butterworth filter with a cutoff frequency of $1000 \mathrm{~Hz}$. This filtered data was then applied to Equation 1 to calculate the SI. Equation 1 is shown below for 
convenience. Due to the fact that the impact surface being used is polycarbonate and is deflecting slightly during the impact, the acceleration curve has multiple peaks. To diminish the effect and to produce more consistent results, the experiments following the screening ANOVA were carried out with a rigid impact surface. The Matlab code used to carry out the SI calculations for the screening analysis can be found in Appendix G:

Representative Matlab Code for Calculating Severity Index.

$$
S I=\int_{0}^{T} A^{2.5} d t
$$

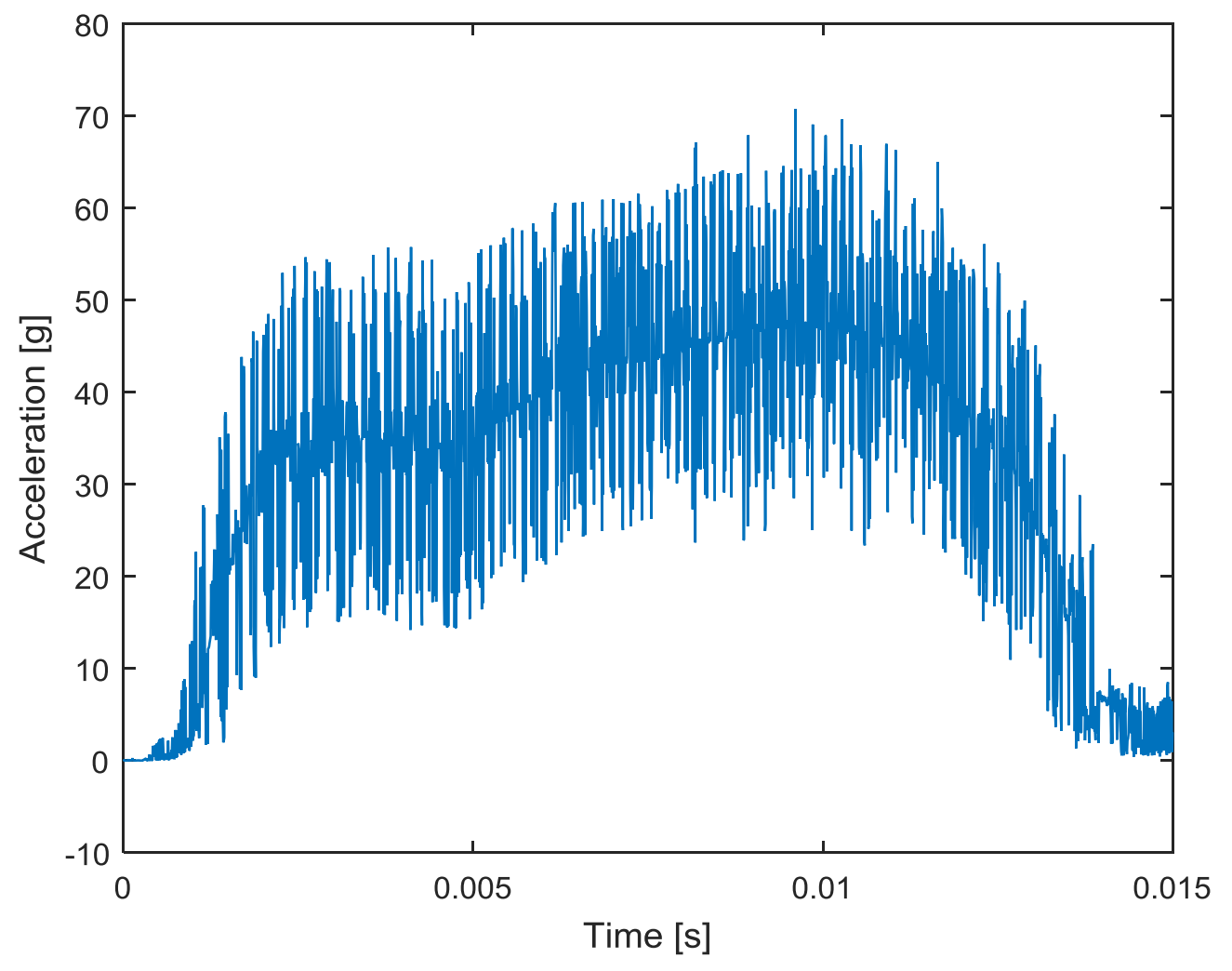

Figure 33. Raw (unfiltered) acceleration data from Abaqus/CAE for Screening ANOVA Run 1. 


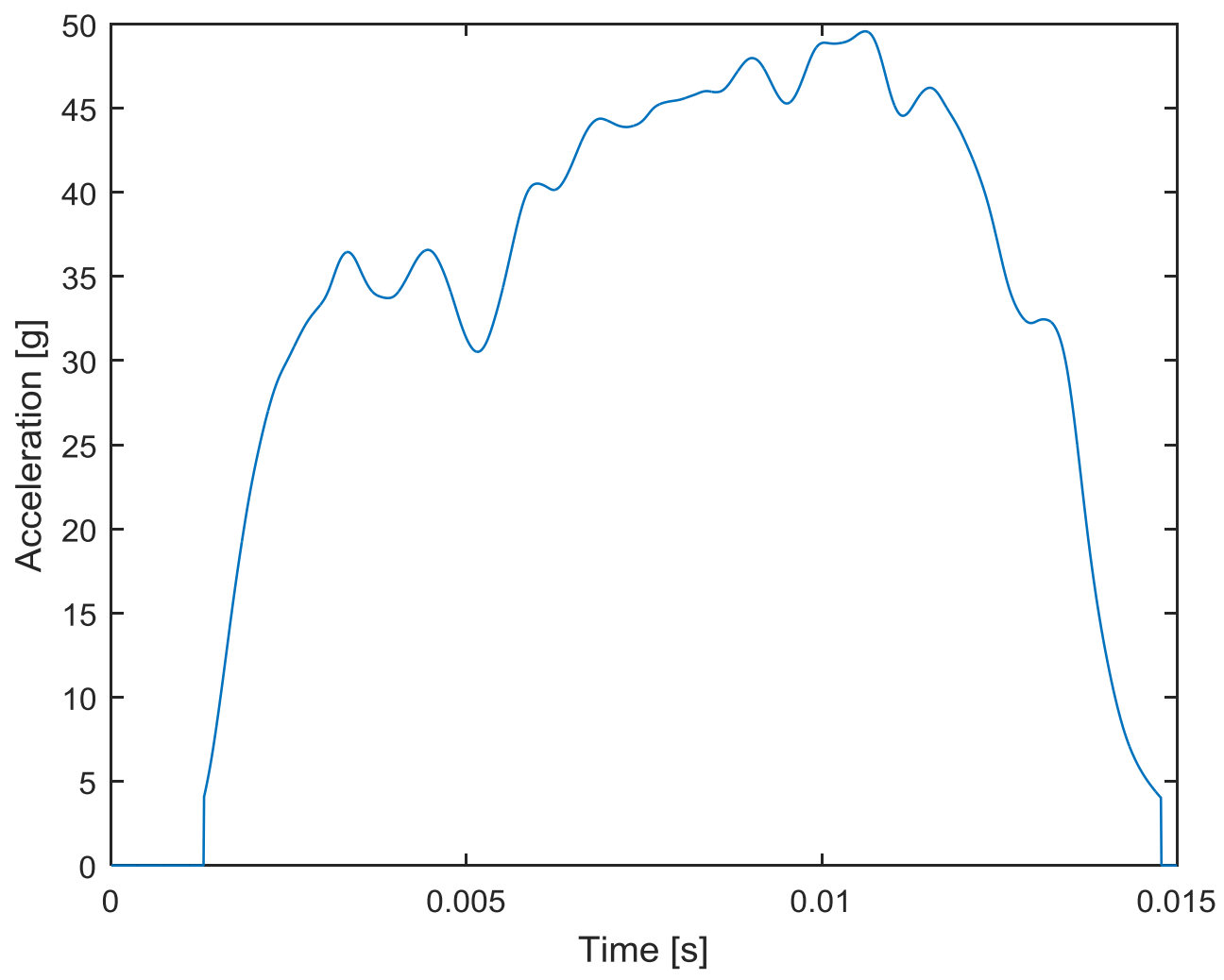

Figure 34. Acceleration data from Screening ANOVA Run 1 filtered with 4-pole low pass Butterworth filter. The acceleration data is cut off at 4 G's to comply with NOCSAE Severity Index calculation standards.

Based on the screening ANOVA it was determined that a design with two oval shaped channels with no cores and a minimum shell thickness of 0.05 " produced the lowest SI. The minimum shell thickness of 0.10 " was chosen to proceed into the following experiments because a shell thickness of 0.05 " would lead to a location of extreme structural weakness that would not be acceptable in real life situations. The factors that will be optimized in the next experiment will be the channel diameter, the channel spacing, and the channel width. The main effects graph indicates that the impact velocity was by far the most significant factor in determining the SI, but since this is not a factor that is controllable through the design alone it has been removed from the proceedings. It is an indication that the statistical analysis is working, however, since the impact velocity 
would intuitively have the largest effect of the severity of the impact. The remaining analyses were completed at $80 \mathrm{in} / \mathrm{s}$.

The sponsor also indicated that they were curious if closing the ends of the channels would have any effect on the impact reduction the helmet provided. After running the trials again with the channels capped, it was determined that there was no difference between the open and capped designs.

\subsection{Follow-up Analysis}

\subsubsection{Round 1}

Once the screening ANOVA study had been run, it was then necessary to optimize the chosen variables (diameter, channel spacing and channel width) to determine the best possible configuration. A list of the factors and levels to be tested in the follow-up ANOVA can be seen in Table 7.

Table 7. Factors and levels for Follow-up ANOVA study.

\begin{tabular}{|c|c|c|c|}
\hline & \multicolumn{3}{|c|}{ Factors } \\
\hline & Diameter & Channel Spacing & Channel Width \\
\hline \multirow{6}{*}{ 吕 } & $.05 "$ & $10^{\circ}$ & $3 / 16^{\prime \prime}$ \\
\hline & $.10 "$ & $17.5^{\circ}$ & 6/16" \\
\hline & $.15 "$ & $25^{\circ}$ & 9/16" \\
\hline & $.20 "$ & $32.5^{\circ}$ & $12 / 16^{\prime \prime}$ \\
\hline & $.25 "$ & $40^{\circ}$ & $15 / 16^{\prime \prime}$ \\
\hline & $.30 "$ & $47.5^{\circ}$ & 18/16" \\
\hline
\end{tabular}

To test such a wide range of levels, the analysis had to be broken up into a number of smaller ANOVA studies. The bold lines in Table 7 above indicate the groupings for these ANOVA analyses. A fractional factorial design was still going to be used to reduce the 
overall number of runs, in this case a $2^{3-1}$ factorial design. Table 8 , Table 9 and Table 10 below indicate the factors and levels for follow-up ANOVA 1, 2 and 3 and Table 11 shows the run scheme for a $2^{3-1}$ factorial design. The factors from each ANOVA that produced the lowest Severity Index would then be advanced to another analysis where they would be tested against the winning factors from the other ANOVA's. This tournament would proceed until there was a single winner for each of the three factors.

Table 8. Run table for the $2^{3-1}$ fractional factorial design for Follow-up ANOVA \#1 analysis.

\begin{tabular}{|c|c|c|c|}
\cline { 3 - 4 } \multicolumn{2}{c|}{} & \multicolumn{2}{c|}{ Levels } \\
\hline$\#$ & Factor & $\mathbf{- 1}$ & $\mathbf{+ 1}$ \\
\hline 1 & Diameter & $.05^{\prime \prime}$ & $.10^{\prime \prime}$ \\
\hline 2 & Channel Spacing & $10^{\circ}$ & $17.5^{\circ}$ \\
\hline 3 & Channel Width & $3 / 16^{\prime \prime}$ & $6 / 16^{\prime \prime}$ \\
\hline
\end{tabular}

Table 9. Run table for the $2^{3-1}$ fractional factorial design for Follow-up ANOVA \#2 analysis.

\begin{tabular}{|c|c|c|c|}
\cline { 3 - 4 } \multicolumn{2}{c|}{} & \multicolumn{2}{c|}{ Levels } \\
\hline$\#$ & Factor & $\mathbf{- 1}$ & $\mathbf{+ 1}$ \\
\hline 1 & Diameter & $.15^{\prime \prime}$ & $.20^{\prime \prime}$ \\
\hline 2 & Channel Spacing & $25^{\circ}$ & $32.5^{\circ}$ \\
\hline 3 & Channel Width & $9 / 16^{\prime \prime}$ & $12 / 16^{\prime \prime}$ \\
\hline
\end{tabular}

Table 10. Run table for the $2^{3-1}$ fractional factorial design for Follow-up ANOVA \#3 analysis.

\begin{tabular}{|c|c|c|c|}
\cline { 3 - 4 } \multicolumn{2}{c|}{} & \multicolumn{2}{c|}{ Levels } \\
\hline$\#$ & Factor & $\mathbf{- 1}$ & $\mathbf{+ 1}$ \\
\hline 1 & Diameter & $.25^{\prime \prime}$ & $.30^{\prime \prime}$ \\
\hline 2 & Channel Spacing & $40^{\circ}$ & $47.5^{\circ}$ \\
\hline 3 & Channel Width & $15 / 16^{\prime \prime}$ & $18 / 16^{\prime \prime}$ \\
\hline
\end{tabular}


Table 11. Run table for a $2^{3-1}$ fractional factorial ANOVA design.

\begin{tabular}{|c|c|c|c|}
\cline { 2 - 4 } \multicolumn{1}{c|}{} & \multicolumn{3}{c|}{ Factor/Level } \\
\hline Run & $\mathbf{1}$ & $\mathbf{2}$ & $\mathbf{3}$ \\
\hline $\mathbf{1}$ & -1 & -1 & +1 \\
\hline $\mathbf{2}$ & +1 & -1 & -1 \\
\hline $\mathbf{3}$ & -1 & +1 & -1 \\
\hline $\mathbf{4}$ & +1 & +1 & +1 \\
\hline
\end{tabular}

\subsubsection{Round 1 Results}

The Severity Index in the follow-up ANOVA was calculated in the same manner as in the screening trials. Table 12 provides the SI values for ANOVAs 1, 2 and 3 respectively. Again, this data was input into Minitab and the main effects plot indicated which level produced the lower SI. Table 13, presents the winning levels from each ANOVA.

Table 12. Severity Index results from follow-up ANOVAs 1, 2 and 3.

\begin{tabular}{|c|c|c|c|}
\cline { 2 - 4 } \multicolumn{1}{c|}{} & ANOVA 1 & ANOVA 2 & ANOVA 3 \\
\hline Run & SI & SI & SI \\
\hline $\mathbf{1}$ & 101.81 & 99.17 & 98.16 \\
\hline $\mathbf{2}$ & 103.08 & 99.05 & 100.45 \\
\hline $\mathbf{3}$ & 103.31 & 98.50 & 96.62 \\
\hline $\mathbf{4}$ & 100.23 & 99.91 & 99.60 \\
\hline
\end{tabular}

Table 13. Factors and winning levels from follow-up ANOVAs 1, 2 and 3.

\begin{tabular}{|l|l|l|l|}
\hline Factor & ANOVA 1 & ANOVA 2 & ANOVA 3 \\
\hline Diameter & $0.10^{\prime \prime}$ & $0.15^{\prime \prime}$ & $.25^{\prime \prime}$ \\
\hline Channel Spacing & $17.5^{\circ}$ & $25^{\circ}$ & $47.5^{\circ}$ \\
\hline Channel Width & $6 / 16^{\prime \prime}$ & $9 / 16^{\prime \prime}$ & $15 / 16^{\prime \prime}$ \\
\hline
\end{tabular}




\subsubsection{Round 2}

The winning levels from ANOVAs 1, 2 and 3 were then combined into two more

ANOVA analyses. These experiments are denoted in Table 14 and Table 15.

Table 14. Run table for the $2^{3-1}$ fractional factorial design for Follow-up ANOVA \#4 analysis.

\begin{tabular}{|c|c|c|c|}
\cline { 3 - 4 } \multicolumn{2}{c|}{} & \multicolumn{2}{c|}{ Levels } \\
\hline$\#$ & Factor & $\mathbf{- 1}$ & $\mathbf{+ 1}$ \\
\hline 1 & Diameter & $.10^{\prime \prime}$ & $.15^{\prime \prime}$ \\
\hline 2 & Channel Spacing & $17.5^{\circ}$ & $25^{\circ}$ \\
\hline 3 & Channel Width & $6 / 16^{\prime}$ & $9 / 16^{\prime}$ \\
\hline
\end{tabular}

Table 15. Run table for the $2^{3-1}$ fractional factorial design for Follow-up ANOVA \#5 analysis.

\begin{tabular}{|c|c|c|c|}
\cline { 3 - 4 } \multicolumn{2}{c|}{} & \multicolumn{2}{c|}{ Levels } \\
\hline$\#$ & Factor & $\mathbf{- 1}$ & $\mathbf{+ 1}$ \\
\hline 1 & Diameter & $.15^{\prime \prime}$ & $.25^{\prime \prime}$ \\
\hline 2 & Channel Spacing & $25^{\circ}$ & $47.5^{\circ}$ \\
\hline 3 & Channel Width & $9 / 16^{\prime \prime}$ & $15 / 16^{\prime \prime}$ \\
\hline
\end{tabular}

\subsubsection{Round 2 Results}

The severity indices for ANOVAs 4 and 5 can be seen in Table 16 below and the winning levels of the tested factors in Table 17.

Table 16. Severity Index results from follow-up ANOVAs 4 and 5.

\begin{tabular}{|c|c|c|}
\cline { 2 - 3 } \multicolumn{1}{c|}{} & ANOVA 4 & ANOVA 5 \\
\hline Run & SI & SI \\
\hline $\mathbf{1}$ & 101.99 & 102.85 \\
\hline $\mathbf{2}$ & 101.21 & 97.05 \\
\hline $\mathbf{3}$ & 99.16 & 100.23 \\
\hline $\mathbf{4}$ & 99.19 & 99.03 \\
\hline
\end{tabular}


Table 17. Factors and winning levels from follow-up ANOVAs 4 and 5.

\begin{tabular}{|l|l|l|}
\hline Factor & ANOVA 4 & ANOVA 5 \\
\hline Diameter & $0.15^{\prime \prime}$ & $0.25^{\prime \prime}$ \\
\hline Channel Spacing & $25^{\circ}$ & $47.5^{\circ}$ \\
\hline Channel Width & $6 / 16^{\prime \prime}$ & $9 / 16^{\prime \prime}$ \\
\hline
\end{tabular}

\subsubsection{Round 3}

The final follow-up ANOVA scheme is listed in Table 18.

Table 18. Run table for the $2^{3-1}$ fractional factorial design for Follow-up ANOVA \#6 analysis.

\begin{tabular}{|c|c|c|c|}
\cline { 3 - 4 } \multicolumn{2}{c|}{} & \multicolumn{2}{c|}{ Levels } \\
\hline$\#$ & Factor & $\mathbf{- 1}$ & $\mathbf{+ 1}$ \\
\hline 1 & Diameter & $.15^{\prime \prime}$ & $.25^{\prime \prime}$ \\
\hline 2 & Channel Spacing & $25^{\circ}$ & $47.5^{\circ}$ \\
\hline 3 & Channel Width & $6 / 16^{\prime}$ & $9 / 16^{\prime \prime}$ \\
\hline
\end{tabular}

\subsubsection{Round 3 Results}

The final follow-up ANOVA yielded the optimized design. The SI values for the four runs in ANOVA 6 are listed below in Table 19. The configuration of geometries that best reduces Severity Index is one in which there are two oval channels each with a diameter of .25" and a width of 6/16" spaced 47.5 degrees off center. The optimized design can be seen in Figure 35. A technical drawing of this configuration can be found in Appendix H: Drawing of the Optimal Helmet Configuration. 
Table 19. Severity Index results from follow-up ANOVA 6.

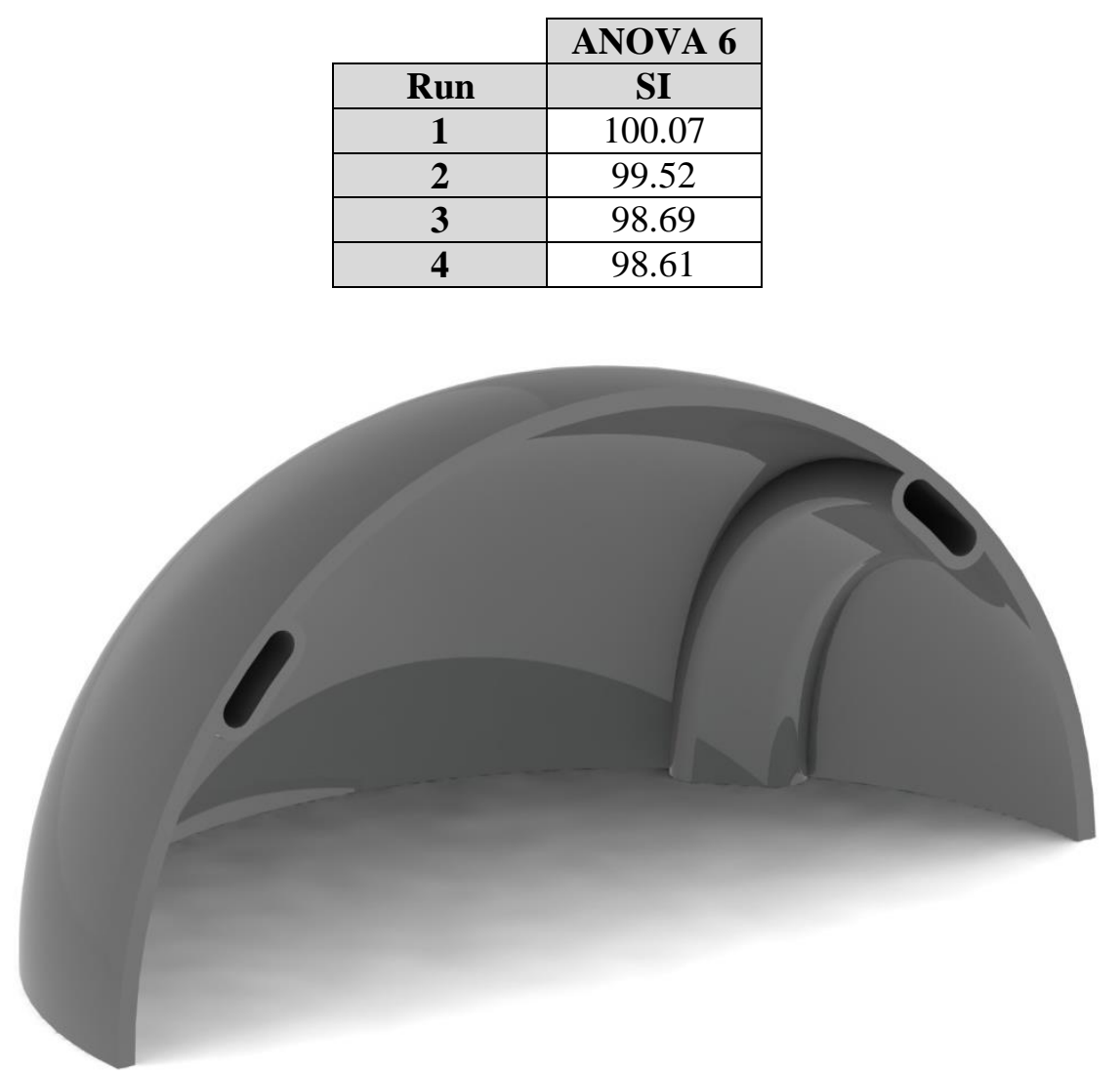

Figure 35. Rendering of the optimal configuration of the impact reduction helmet.

\subsection{Additional Simulations}

After determining the optimized design, it was now necessary to run a simulation that utilized the parameters of this design to compare the resulting SI to the control simulation. In addition to the optimized design, a simulation with a third channel directly in the middle was also tested. Up until this point only even numbers of channels had been tested so a trial was done by adding a third identical channel to the middle of the optimized design. The results from these trials can be seen below in Table 20. It can be seen that the two channeled design has a lower Severity Index value and is, therefore, the better design configuration. 
Table 20. Final Analysis Severity Index results. Includes the optimized design and a three channeled design using the optimized channel geometry.

\begin{tabular}{|c|c|}
\cline { 2 - 2 } \multicolumn{1}{c|}{} & Final Analysis \\
\hline Run & SI \\
\hline Optimized Design & 99.73 \\
\hline Three Channel Design & 101.29 \\
\hline
\end{tabular}

\subsection{Testing}

Based on the results from the final simulations, the design that best reduces Severity Index is one that conforms to the original optimized configuration. The last step was to test this design in real life on an impact testing machine to complete the validation of the model. Masters student Michael Schuster developed a helmet testing machine for this purpose. [22] This testing machine can be seen in Figure 36. The helmet itself was 3-D printed due to its complicated geometry. A fused deposition modeling ABS plastic printing method was chosen due to price constraints. 


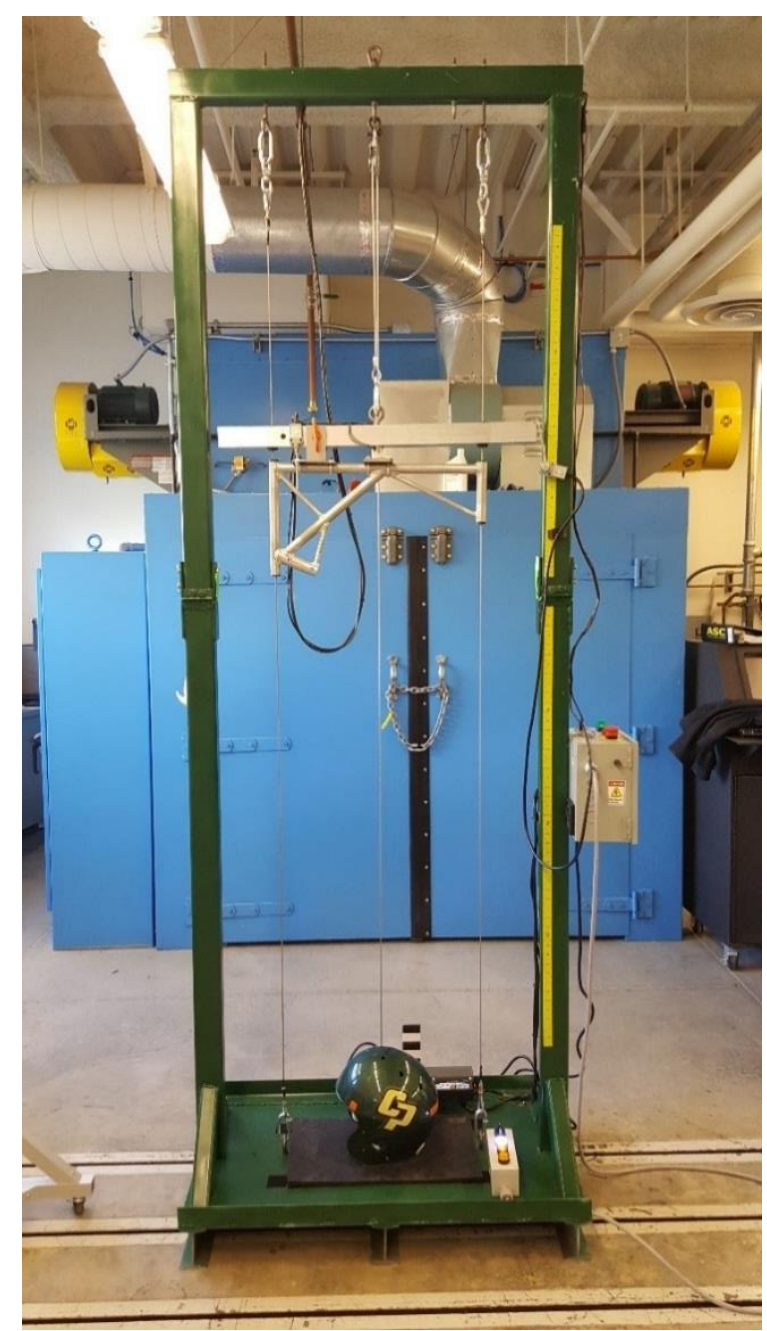

Figure 36. Impact testing machine used to test the 3-D printed control and channeled helmets. [22]

Because the "optimized" design seemed to move the channels as far as possible from the area of impact, it is clear that the simulations show that the best geometry is one that most closely resembles the control helmet. This is possibly because having a channel under the impact zone effectively reduces the thickness of the padding at that location. It is possible that Abaqus is not able to capture how the channels themselves are helping, however. Physically testing a design in which the channels are as far removed from the impact as possible does not help determine whether they help or not in real life. Because of this a separate design for the 3-D printed experimental model was chosen that brought the 
channels back into play. The geometries of the channels from the optimized design was carried into this design, but the spacing and number of channels was altered. The tested experimental helmet had four channels spaced 30 degrees from each other. The final printed models can be seen in Figure 37, Figure 38 and Figure 39 below. The outer geometry matches that of a traditional football helmet.

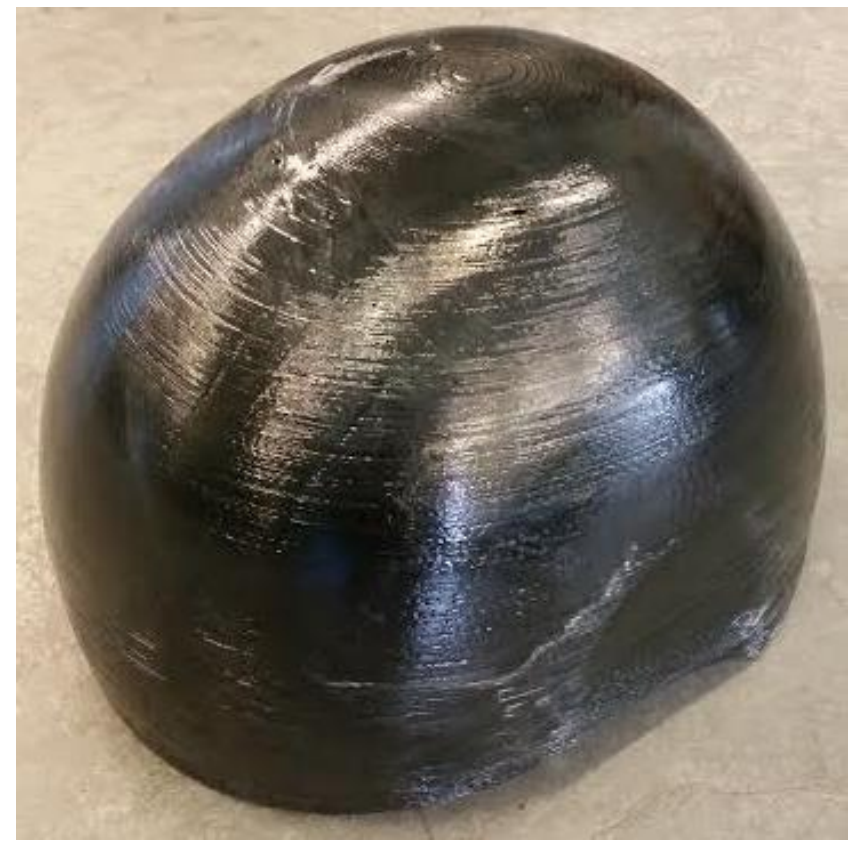

Figure 37. Outer side of the control and channeled 3-D printed football helmets. Both designs utilized the same outer geometries. 


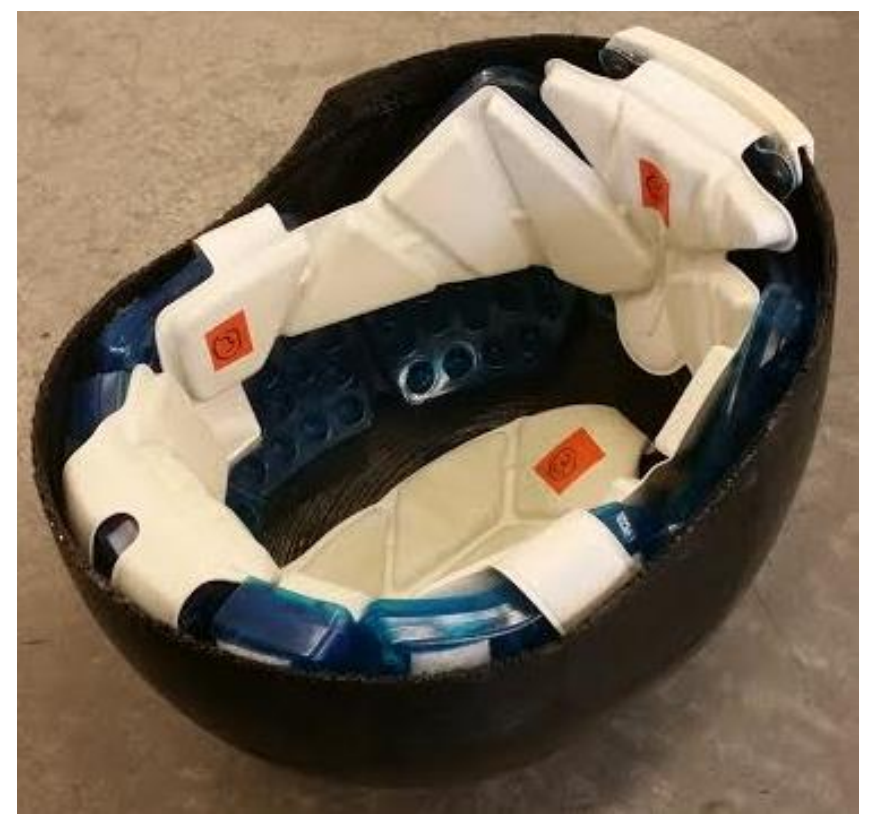

Figure 38. 3-D printed control football helmet used to compare against the channeled design.

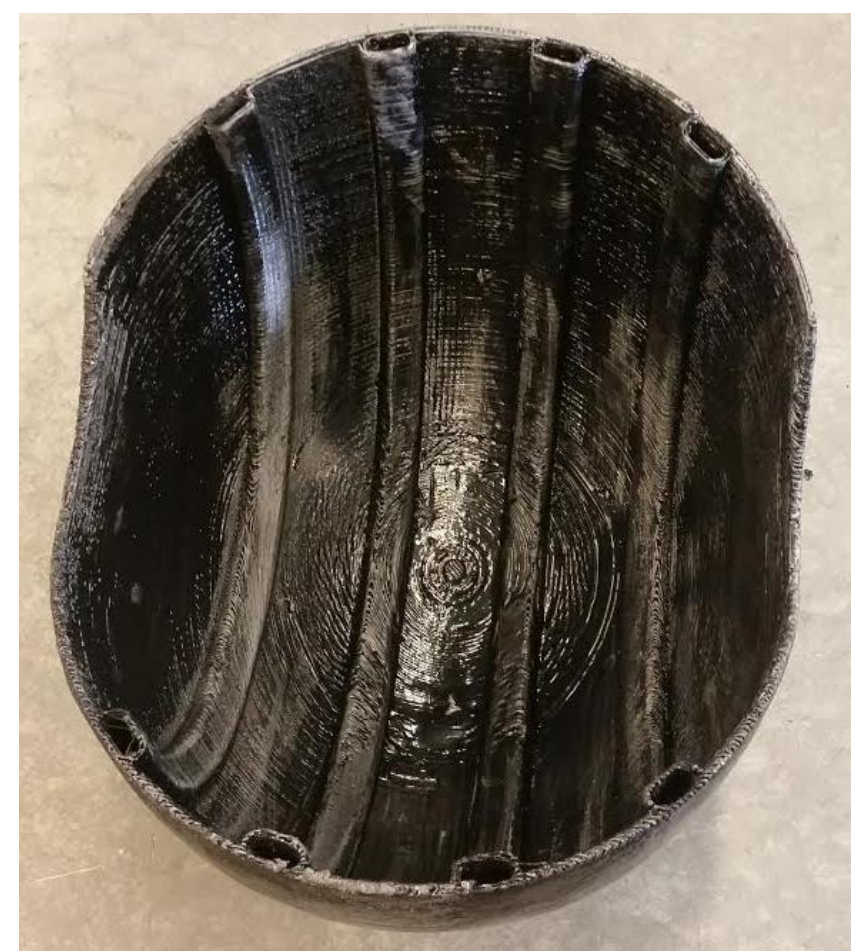

Figure 39. 3-D printed impact diffusion helmet design. 
The testing itself consisted of impacting a real football helmet and the two 3-D printed helmets using the impact rig. The normal football helmet was tested in order to provide assurance that the acceleration data that was collected was consistent across the tests. This calibration data was consistent to within $14 \%$ indicating that the impact tester was able to perform with enough accuracy to obtain usable experimental results. For more information on this topic, reference Physical Testing of Potential Football Helmet Design Enhancements by Michael Schuster. At this point the control helmet was loaded onto the tester and impacted from 2 feet a total of five times. The speed of the impact was determined through the use of a high speed camera and was calculated to be $159 \mathrm{in} / \mathrm{s}$ for drops from this height. The helmet was then raised to an impact height of 4 feet, but cracked after the first drop. The channeled helmet was then impacted from 2 feet five times and then raised to 4 feet where it too cracked on the first drop. Appendix I: High Speed camera screen captures of the first control helmet impact test show a series of frame captures from the high speed camera during the first impact trial of the control helmet.

It should be noted that the channeled design did not included sub-padding like the Abaqus simulations did. This was done to provide a more direct correlation between the channeled and control designs. If sub-padding were to be added to the channeled design, it would provide that model with additional damping potential. It was important to keep the comparison between the two designs as simple as possible so a conclusion could be drawn about the channels and not about the extra padding that was included between the channels. 
Several additional Abaqus simulations were carried out at this stage to verify the earlier results of the FEA. The control geometry that was 3-D printed was modeled with ABS plastic as the shell material and analyzed similarly to the previously run simulations. Having these results allowed for a direct comparison between real-world impacts and the simulations that were used to optimize the helmet design and was extremely important in authenticating the optimization simulations. 


\section{Results}

The results of the simulation trials are very clear. They plainly indicate that a helmet design that moves the channel geometries as far away from the impact zone as possible is the optimal design. As discussed briefly in the Testing section above, this has a fairly intuitive explanation. By having channels that extend below the surface of the shell, the design reduces the thickness of the padding under the impact zone. Moving the channels away from the impact as much as possible allows the maximum padding to exist between the impact and the head. Additionally, the channels provided the shell with a further measure of stiffness that could account for the heightened SI. This was further corroborated when a channel was placed directly below the impact location and it performed worse than the optimized design. If there is a mechanism that would help dissipate energy, the Abaqus simulations did not capture it and instead performed exactly as one would expect. In addition, the "optimized" design still produced a higher Severity Index than the control helmet.

The physical testing was able to further illuminate the effect of the channels. After comparing the average Severity Index results from the control and channeled designs, it was evident that the channels were not doing anything to help with the impact. In fact the channels seem to be exacerbating the impact and increasing the SI. Table 21 below shows the calculated Severity Index for the control helmet and the experimental helmet. A number of other impact metrics are included here to further support the findings. The Severity Index values are the values for the Severity Index from the time that acceleration goes above 4 g's to when it falls below 4 g's. The windowed Severity Index value 
truncates the data to $25 \mathrm{~ms}$ corresponding to the time that the helmet was in contact with the impact surface, determined through analyzing the high speed camera footage. Head injury criterion (HIC) 15 and 36 are calculated similarly to the SI, but for varying lengths of time and with a slightly different integral. HIC 15 is measured as the greatest value of the equation over a $15 \mathrm{~ms}$ time interval and HIC 36 is the largest integral value achieved over any 36 ms time period. Equations 11 and 12 below indicate the HIC 15 and HIC 36 criteria. Figure 40 and Figure 41 show the difference between the traditional SI calculation and the truncated results used to calculate HIC.

$$
\begin{aligned}
& H I C_{15}=\left\{\left[\frac{1}{t_{2}-t_{1}} \int_{t_{1}}^{t_{2}} a(t) d t\right]^{2.5}\left(t_{2}-t_{1}\right)\right\}_{\max } \text { for }\left(t_{2}-t_{1}\right)=0.015 \mathrm{~s} \\
& H I_{36}=\left\{\left[\frac{1}{t_{2}-t_{1}} \int_{t_{1}}^{t_{2}} a(t) d t\right]^{2.5}\left(t_{2}-t_{1}\right)\right\}_{\max } \text { for }\left(t_{2}-t_{1}\right)=0.036 \mathrm{~s}
\end{aligned}
$$

Table 21. Impact metric values for the 3-D printed control and channeled helmet designs averaged over five runs.

\begin{tabular}{|c|c|c|}
\hline Impact Metric & Control Helmet & Channeled Helmet \\
\hline Severity Index (1200 = FAIL) & 326.90 & 878.52 \\
\hline Windowed Severity Index & 275.65 & 811.11 \\
\hline HIC 15 (700 = FAIL) & 169.60 & 436.46 \\
\hline HIC 36 (1000 = FAIL) & 167.85 & 456.13 \\
\hline Max Acceleration (300 G's = FAIL) & $88.92 \mathrm{G}$ & $173.56 \mathrm{G}$ \\
\hline
\end{tabular}




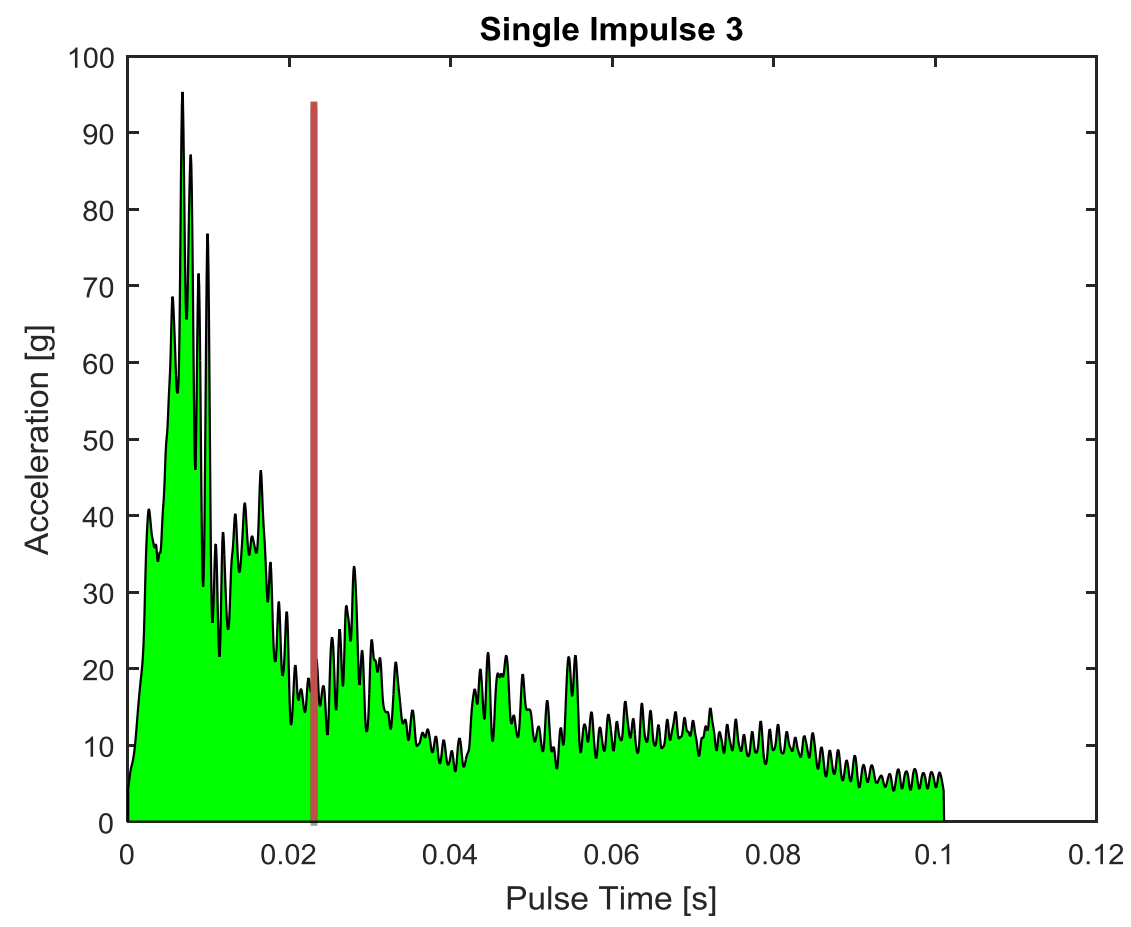

Figure 40. Plot of the filtered acceleration pulse from the third drop of the control helmet. This plot shows the entire duration of the impact that exists above $4 \mathrm{G}$ 's. Red line indicates where contact with impact pad ended.

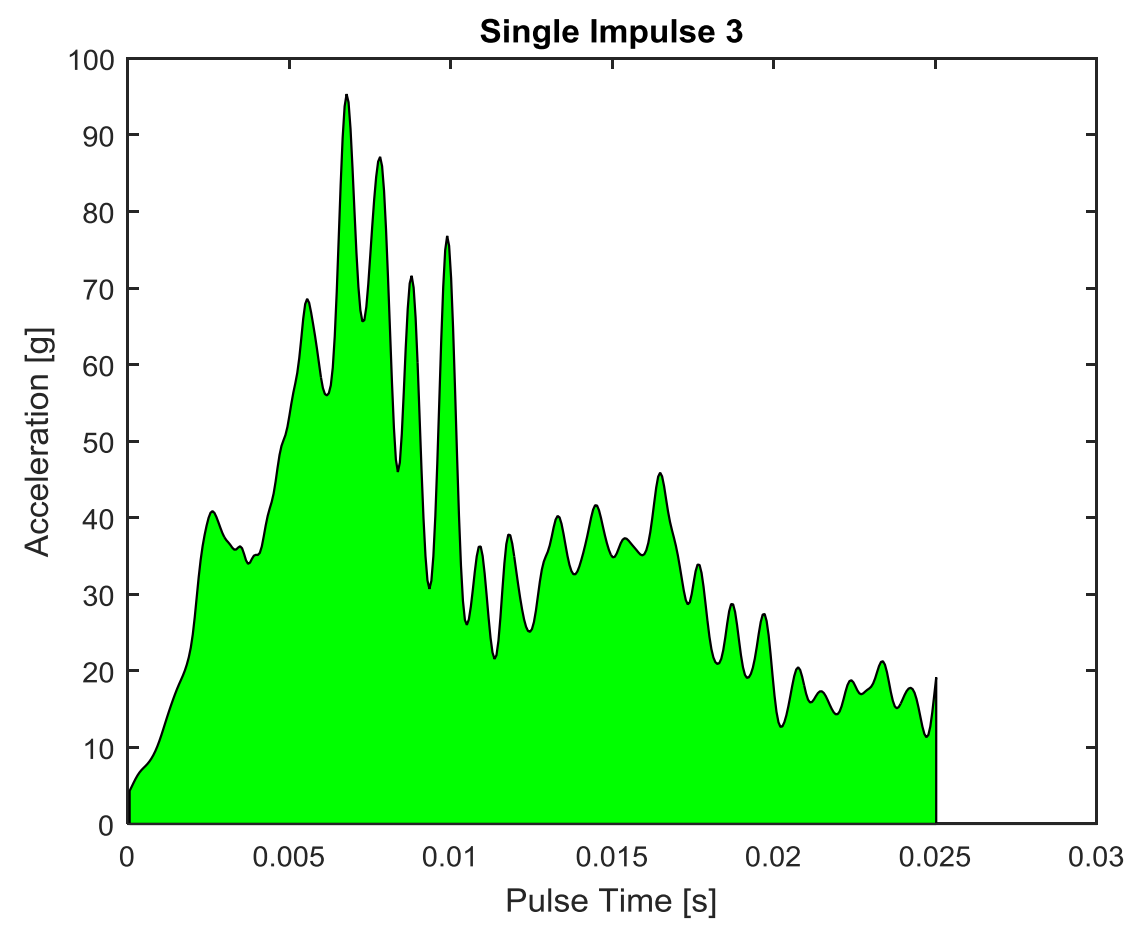

Figure 41. Plot of the filtered acceleration data from the third drop of the control helmet.

This plot only shows up to $25 \mathrm{~ms}$ pulse time since that was the duration of impact determined through studying the high speed camera footage. 
After attempting to run a simulation identical to the 2-ft drop of both the control helmet and the experimental helmet, it was determined that the padding properties were not able to withstand that high of an impact speed. In order to still achieve verification, a 1-ft drop of the polycarbonate helmet was performed on the impact rig and a similar Abaqus simulation was generated. The simulation indicated that the Severity Index of the helmet impact should be 140 . The physical testing resulted in an SI value close to 510 . This huge disparity between the two values can be explained by the movement of the carriage in the non-vertical direction. When only considering the acceleration in the vertical direction, the SI of the physical test drops to 161 . This is $13.9 \%$ different than the simulation predicted. Since this is an impact event, this percent difference is small enough to mean that the optimization simulations can be considered validated since the same parameters were used in this simulation as the earlier ones.

Based on the results from the optimization trials as well as from the physical testing, the impact diffusion helmet design has been shown to produce a higher SI than that of a traditional helmet design. 


\section{Discussion}

This project presented a number of interesting concepts to be studied. First of all, the channeled structure was remarkable in that it was completely novel and nothing like it

preceded it. Unfortunately, it did not respond as anybody would have hoped and instead produced a higher SI than the control helmet in the physical testing. This may be explained by helmets used for the testing. Since ABS plastic was used for the full size prototypes, it is difficult to correlate those results to what would happen to a polycarbonate helmet. Additionally, the channeled prototype used was subjected to an additional surface treatment that was not performed on the control helmet. This caused the layers of the 3-D print to adhere much more effectively on the experimental helmet which could cause additional stiffness in this model. A further problem with the experimental helmet was that a portion of the channels near the crown of the helmet was filled in with support material. This could have reduced the ability of the helmet to perform under loading. A final possible source of discrepancy was the elimination of padding between the channels in the full scale testing. This was done in order to specifically test the ability of the channels, but since the impact diffusion helmet design contains this padding, it may have been appropriate to include in the testing. Adding the sub-padding to the physical model is a test that would have been performed had the helmets not had such a short lifespan. Since that test was never conducted, however, there is no data regarding how the helmet would behave with it included.

By far the most time consuming and thought provoking portion of the project was the model development phase. It involved generating a working helmet model that accurately 
represented a real world impact. Despite all the work that went into this stage, there are a number of things that could have made this a more accurate model. All of these would complicate the simulation and increase solve time, but more life-like results would have been achieved. The first and most important of these changes would be to change the padding properties. The padding properties that ended up being a part of the final simulations were modified data from experiments of car bumper foam. It would be better to have a padding that had a real-world analogue so that the properties could be separately verified and tested. In future simulations, it would probably be best to directly test the Schutt Air XP padding for material properties and then apply those to the padding models. Additionally, the padding properties were calibrated in the small scale testing by comparing the test results to the simulations. The testing involved impacting the test specimen on a rubber mat. The simulation impacted on a rigid surface. This caused the additionally damping from the rubber mat to be convoluted with the helmet damping. When calibrating the padding, a rubber mat should have been used to mimic the physical testing. Another possible area of improvement for the simulations would be to use more accurate helmet geometries. The simulations run for the analysis modeled the shell of the helmets as perfectly spherical. While there may not be a significant difference in the results of the simulations with the two different shell types, it would bring the models closer to real life. Along this same line, traditional football helmets cover the player's ears as well as the top of the head. There is likely a component of friction from the sides of the player's head during an impact. This friction was neglected in the analysis in favor of the much larger damping forces of the padding, but it would be a good place to improve the model. 
The statistical analysis of the FEA models deserved more thought. The optimization trial involved performing several different two factor ANOVA studies. It would have been better to run these studies as three factor ANOVA studies in order to be able to see curvature in the Severity Index results. It's also possible that a more complicated statistical design would allow the optimization to be conducted with more accuracy than a tournament style analysis.

The physical testing also had a few problems that should be addressed before any further testing resumed. First, it was unfortunate that both 3-D printed helmet cracked so soon after the testing began. This presented only a small pool of data to draw from to make conclusions. A more professionally manufactured set of prototype helmets would be necessary to continue testing of this design. Testing the helmets from the several drop heights would definitely make the conclusions more concrete. Secondly, it was very difficult to accurately determine the duration of the impact. NOCSAE specifies that the SI calculation window should remain open until the acceleration falls below 4 G's. The biggest problem with this is that after the impact has completed, the carriage assembly that holds the helmet rocks back and forth. This motion contributes to the acceleration after the impact and lengthens the duration over which the Severity Index is calculated. It may be possible to tighten the guide wires to reduce the movement of the carriage after the impact or perhaps add an accelerometer to the base of the impact tower to record the impact time. 
Lastly, the final simulations that were run to verify the Abaqus simulations to the fullscale testing presented a few problems. Initially the padding component of the simulation became very unstable at the 2 -ft impact speed. The increased speed of the impact caused the headform to intrude all the way through the padding until it touched the inner shell. This is likely due to how the properties for the padding were initially developed. To get these properties in the first place, the Ford bumper foam data were scaled until the simulated results matched the small scale testing results. This mode of gathering material model parameters seems to have misrepresented the strain rate sensitivity of the padding. One would expect the stiffness of the padding to go up as the strain rate increased, but this appears not to have happened to the degree necessary to accurately capture acceleration data for higher drop speeds. To alleviate this concern, further full-scale testing was done with a traditional polycarbonate football helmet at much lower impact speeds. This test data was then paired with Abaqus data that mimicked those tests. The results from these tests were successfully able to verify the Abaqus models. 


\section{Conclusion}

By utilizing finite element analysis methodology, the impact diffusion helmet was able to be fully analyzed and compared to a traditional football helmet. The Abaqus FEA models were initially validated using a variety of methods including comparisons to published correlations and physical testing. The screening series of Abaqus simulations were able to show that two oval shaped channels would lead to the lowest Severity Index. The next series of optimization simulations demonstrated that the best helmet configuration required the channels to be as far from the impact zone of the helmet as possible. Full scale physical testing of a 3-D printed prototype indicated that the channeled design produced a higher Severity Index value from the same drop height as a control helmet. With the results from the simulations and the physical testing in mind, it can be definitively stated that the impact diffusion helmet has a negative effect on the concussion reducing properties of a football helmet. 


\section{WORKS CITED}

1. "Brain Injury Basics." Centers for Disease Control and Prevention. Centers for Disease Control and Prevention, 16 Feb. 2015. Web. 15 Nov. 2015. Web.

2. Broglio, Steven P., Brock Schnebel, Jacob J. Sosnoff, Sunghoon Shin, Xingdong Feng, Xuming He, and Jerrad Zimmerman. "Biomechanical Properties of Concussions in High School Football." Medicine \& Science in Sports \& Exercise 42.11 (2010): 2064-071. Web.

3. Marshall, Stephen W., Kevin M. Guskiewicz, Viswanathan Shankar, Michael Mccrea, and Robert C. Cantu. "Epidemiology of Sports-related Concussion in Seven US High School and Collegiate Sports." Inj. Epidemiol. Injury Epidemiology 2.1 (2015): n. pag. Web.

4. Rowson, Steven, and Stefan M. Duma. "Brain Injury Prediction: Assessing the Combined Probability of Concussion Using Linear and Rotational Head Acceleration." Annals of Biomedical Engineering Ann Biomed Eng41.5 (2013): 873-82. Web.

5. "NFL Acknowledges, for First Time, Link between Football, Brain Disease."ESPN. ESPN Internet Ventures, n.d. Web. 22 Dec. 2015.

6. Yoganandan, Narayan, Frank A. Pintar, and Sanford J. Larsen. "Frontiers in Head and Neck Trauma: Clinical and Biomechanical." PsycEXTRA Dataset (n.d.): n. pag. Web.

7. Standard Test Method and Equipment Used in Evaluating the Performance Characteristics of Headgear/Equipment. Tech. Vol. NOCSAE DOC 001-13m15c. N.p.: National Operating Committee on Standards for Athletic Equipment, 2015. Print.

8. Standard Performance for Newly Manufactured Football Helmets. Tech. Vol. NOCSAE DOC 002-13m13. N.p.: National Operating Committoo on Standards for Athletic Equipment, 2013. Print.

9. Standard Performance Specifications for Recertified Football Helmets. Tech. Vol. NOCSAE DOC 004-11m15. N.p.: National Operating Committoo on Standards for Athletic Equipment, 2015. Print.

10. Rowson, Steven, and Stefan M. Duma. "Development of the STAR Evaluation System for Football Helmets: Integrating Player Head Impact Exposure and Risk of Concussion." Annals of Biomedical Engineering Ann Biomed Eng 39.8 (2011): 2130-140. Web.

11. "Anatomy of a Traditional Football Helmet." The New York Times. The New York Times, 19 Oct. 2010. Web.

12. Bartoli, Ivan, Alessandro Marzani, Francesco Lanza Di Scalea, and Erasmo Viola. "Modeling Wave Propagation in Damped Waveguides of Arbitrary Crosssection." Journal of Sound and Vibration 295.3-5 (2006): 685-707. Web. 
13. Stepanenko, Dmitry A., and Vladimir T. Minchenya. "Modeling of Flexible Waveguides for Ultrasonic Vibrations Transmission: Longitudinal and Flexural Vibrations of Non-deformed Waveguide." Ultrasonics 50.3 (2010): 424-30. Web. 14. "Simulia." Abaqus/CAE User's Guide. Dassault Systemes, 2014. Web.

15. Reeves, Jacob, Keoni Sanchez, and Alek Squires. Impact Diffusion Helmet. Rep. San Luis Obispo: Cal Poly, 2015. Print.

16. Bradtmiller, Bruce, and Martin Friess. A HEAD-AND-FACE ANTHROPOMETRIC SURVEY OF U.S. RESPIRATOR USERS. Rep. N.p, n.d. Anthrotech, 28 May 2004. Web

17. "Solidworks 2016 Help." Solidworks Web Help. Dassault Systemes, 2016. Web.

18. Roark, Raymond J., and Warren C. Young. Roark's Formulas for Stress and Strain. New York: McGraw-Hill, 1989. Print.

19. "Schutt Sports XP Padding." PRWeb. Schutt Sports, n.d. Web. 03 Apr. 2016.

20. "Classification System for Thermoplastic Polyurethane Materials (TPU)." (n.d.):

n. pag. PU Solutions Elastogran. BASF. Web.

21. Geier, Peggy. Polyurethane Foam as an Energy Absorber in Bumper Systems.

N.p.: Ford Motor Compary, 1995. Print.

22. Schuster, Michael. Physical Testing of Potential Football Helmet Design

Enhancements. Master's Thesis, 2016. Print.

23. "NIST/SEMATECH E-Handbook of Statistical Methods." NIST/SEMATECH EHandbook of Statistical Methods. US Department of Commerce, 30 Oct. 2013. Web. 16 Jan. 2016.

24. Onyiah, Leonard C. Design and Analysis of Experiments: Classical and Regression Approaches with SAS. Boca Raton: Taylor and Francis Group, LLC, 2009. Print.

25. "Minitab 17 Help." Minitab. Minitab Statistical Software, n.d. Web. 2016. 


\section{APPENDICES}

Appendix A: Control Helmet Drawing

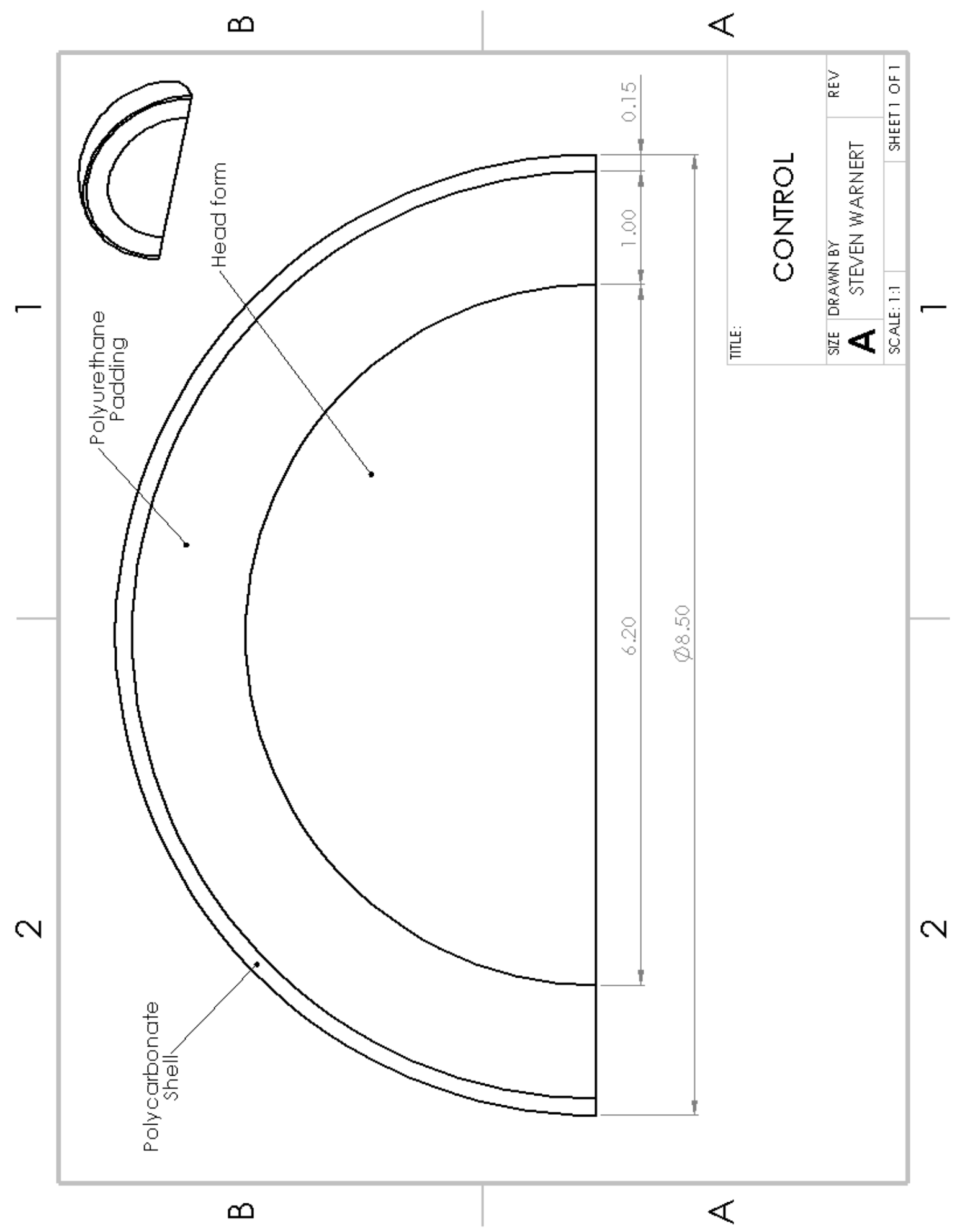




\section{Appendix B: Properties used in Abaqus/CAE simulations}

Elements and Seed sizes:

\begin{tabular}{|c|c|c|c|}
\hline Part & Element Type & Seed size & $\begin{array}{c}\text { Approximate number of } \\
\text { elements }\end{array}$ \\
\hline Impact Surface & $\begin{array}{c}\text { Shell, Explicit, Linear, } \\
\text { Reduced Integration } \\
\text { (S4R) }\end{array}$ & 0.25 & 6,400 \\
\hline Helmet Shell & $\begin{array}{c}\text { 3D Stress, Explicit, } \\
\text { Linear (C3D8) }\end{array}$ & 0.075 & 40,000 \\
\hline Sub-padding & $\begin{array}{c}\text { 3D Stress, Explicit, } \\
\text { Linear (C3D8) }\end{array}$ & 0.25 & 1,000 \\
\hline Cores & $\begin{array}{c}\text { 3D Stress, Explicit, } \\
\text { Linear (C3D8) }\end{array}$ & 0.125 & 5,500 \\
\hline Helmet Padding & $\begin{array}{c}\text { 3D Stress, Explicit, } \\
\text { Linear (C3D8) }\end{array}$ & 0.20 & 3,200 \\
\hline Headform & $\begin{array}{c}\text { 3D Stress, Explicit, } \\
\text { Linear (C3D8) }\end{array}$ & 0.25 & 5,500 \\
\hline
\end{tabular}

\section{Material Properties:}

1. Helmet Padding:

a. Density: 8.22774E-6

b. Low Density Foam:

i. Strain rate measure: Principal

ii. $\mathrm{mu} 0=0.0001$

iii. $\mathrm{mu} 1=0.005$

iv. alpha $=2$

c. Uniaxial Compression Data: 


\begin{tabular}{|r|r|r|}
\hline $\begin{array}{r}\text { Nominal } \\
\text { Stress }\end{array}$ & $\begin{array}{r}\text { Nominal } \\
\text { Strain }\end{array}$ & $\begin{array}{r}\text { Nominal } \\
\text { Strain } \\
\text { rate }\end{array}$ \\
\hline 0 & 0 & 88 \\
\hline 12.69 & 0.05 & 88 \\
\hline 12.69 & 0.1 & 88 \\
\hline 12.69 & 0.15 & 88 \\
\hline 14.505 & 0.2 & 88 \\
\hline 16.3175 & 0.25 & 88 \\
\hline 18.13 & 0.3 & 88 \\
\hline 19.9425 & 0.35 & 88 \\
\hline 21.755 & 0.4 & 88 \\
\hline 23.5675 & 0.45 & 88 \\
\hline 27.195 & 0.5 & 88 \\
\hline 30.82 & 0.55 & 88 \\
\hline 36.26 & 0.6 & 88 \\
\hline 47.1375 & 0.65 & 88 \\
\hline 65.2675 & 0.7 & 88 \\
\hline 108.7775 & 0.75 & 88 \\
\hline 126.9075 & 0.77 & 88 \\
\hline & & \\
\hline
\end{tabular}

d. Uniaxial tension data

\begin{tabular}{|r|r|r|}
\hline $\begin{array}{r}\text { Nominal } \\
\text { Stress }\end{array}$ & $\begin{array}{r}\text { Nominal } \\
\text { Strain }\end{array}$ & $\begin{array}{r}\text { Strain } \\
\text { rate }\end{array}$ \\
\hline 0 & 0 & 0 \\
\hline
\end{tabular}

2. Polycarbonate

a. Density: 0.000112

b. Elastic

i. Young's Modulus: 320,000

ii. Poisson's ratio: 0.37

Step:

1. Time period: $0.015 \mathrm{~s}$

2. Nlgeom: ON

\section{Interactions:}

1. Head to Padding: Surface-to-Surface (Explicit)

2. Padding to Shell: Surface-to-Surface (Explicit)

3. Shell to Wall: General Contact (Explicit) 


\section{Interactions Properties:}

1. Impact
a. Normal Behavior
b. Hard contact
c. Default constraint enforcement method
d. Allow separation after contact

\section{Constraints}

1. Rigid Head

a. Reference point

2. Subpadding to padding

3. Subpadding to shell

4. Cores to channels

\section{Boundary Conditions}

1. Encastre impact surface

2. Symmetry on top surface

\section{Predefined Fields}

1. Velocity in $-\mathrm{V} 3$ direction 


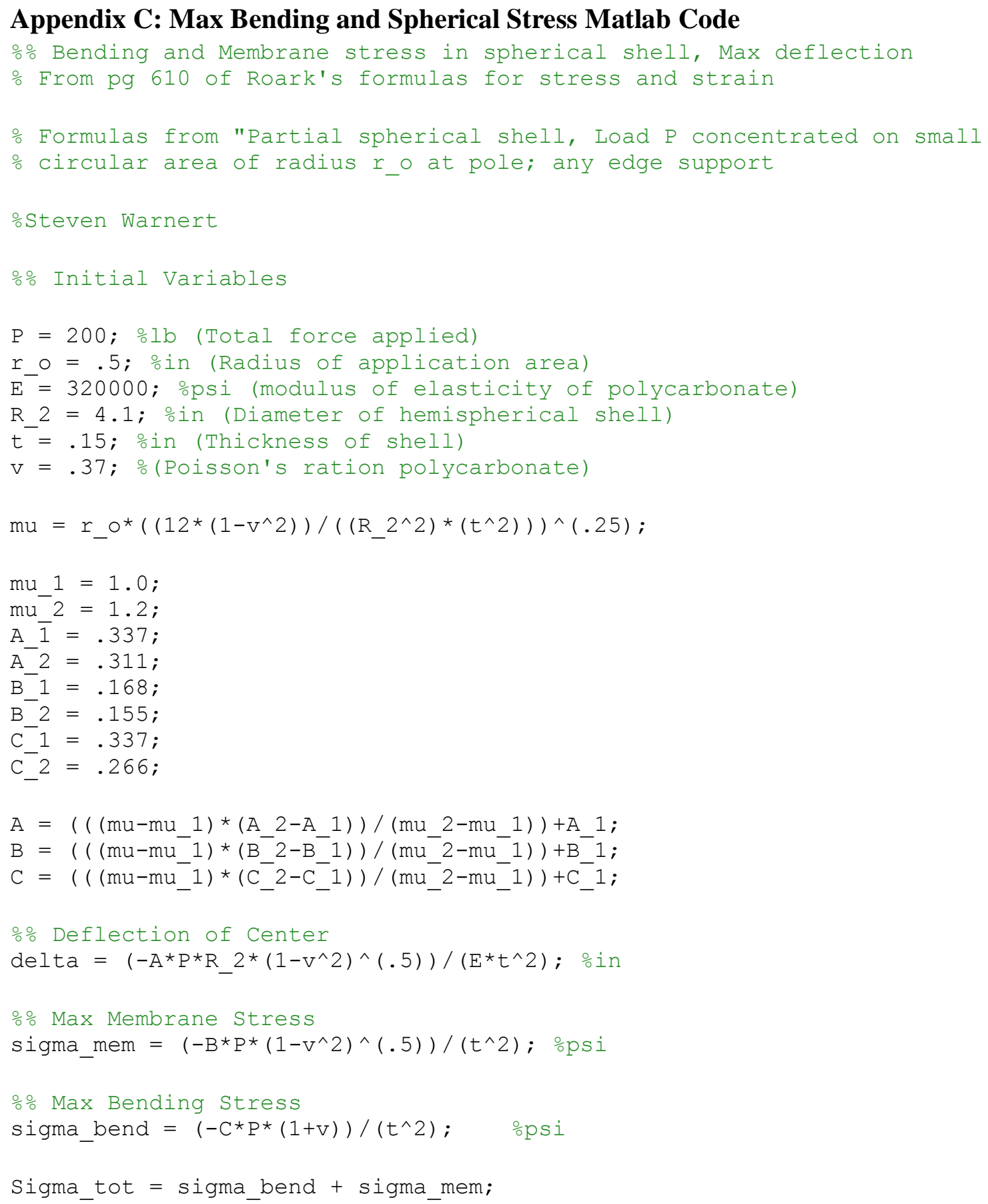




\section{Appendix D: Thermoplastic Polyurethane Material Properties}

\section{Elastollan C $85 \mathrm{~A}$}

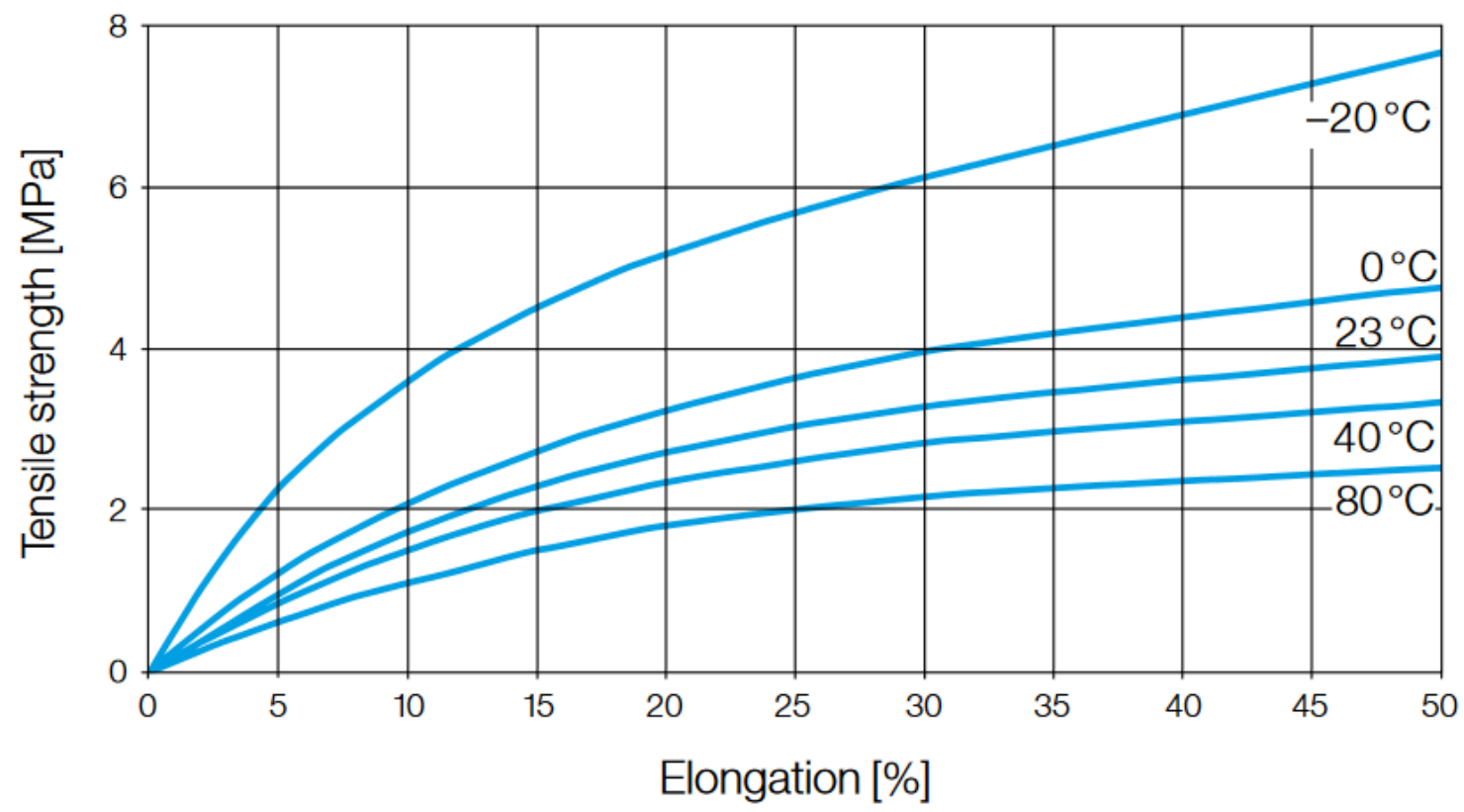

Fig. 24

Figure 42. Graphical representation of stress and strain data for thermoplastic formed polyurethane. The data curve used is the one at $23^{\circ} \mathrm{C}$.

Table 22. Stress and strain data for thermoplastic formed polyurethane at $23^{\circ} \mathrm{C}$ taken from the above plot.

\begin{tabular}{|c|c|}
\hline Strain & Stress \\
\hline 0.025 & 217.56 \\
\hline 0.05 & 304.6 \\
\hline 0.1 & 478.63 \\
\hline 0.15 & 623.66 \\
\hline 0.2 & 812.21 \\
\hline 0.25 & 841.22 \\
\hline 0.3 & 884.73 \\
\hline 0.35 & 906.49 \\
\hline 0.4 & 1015.3 \\
\hline 0.45 & 1044.27 \\
\hline
\end{tabular}




\section{Appendix E: Low Density Foam Material Properties}

\section{ApPendix D.2}

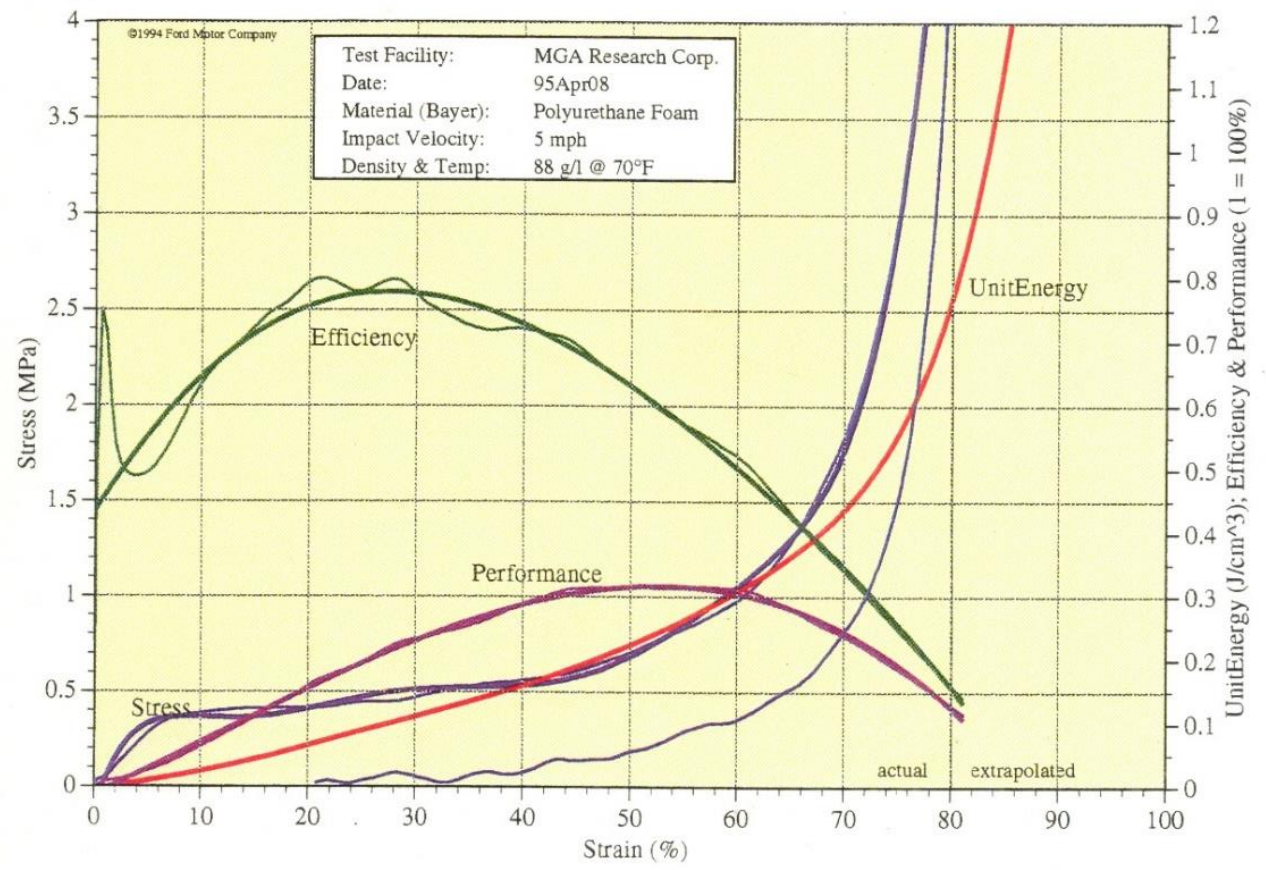

Stress as a Function of Strain, Polyurethane, $88 \mathrm{~g} /, 70^{\circ} \mathrm{F}\left(20^{\circ} \mathrm{C}\right)$

Figure 43. Stress strain data from the Ford motor company handbook on impact reducing foams. The important curve here is the "Stress" curve in blue.

Table 23. Table representation of the stress and strain data taken from the Ford motor company handbook on impact reducing foams.

\begin{tabular}{|c|c|c|c|c|c|c|c|c|c|}
\hline Strain & 0 & 0.05 & 0.10 & 0.15 & 0.20 & 0.25 & 0.30 & 0.35 & 0.40 \\
\hline Stress [psi] & 0 & 50.76 & 50.76 & 50.76 & 58.02 & 65.27 & 72.52 & 79.77 & 87.02 \\
\hline Strain & 0.45 & 0.50 & 0.55 & 0.60 & 0.65 & 0.70 & 0.75 & 0.77 & \\
\hline Stress [psi] & 94.27 & 108.8 & 123.3 & 145 & 188.6 & 261.1 & 435.1 & 507.6 & \\
\hline
\end{tabular}




\section{Appendix F: Drawing of Representative Experimental Model}

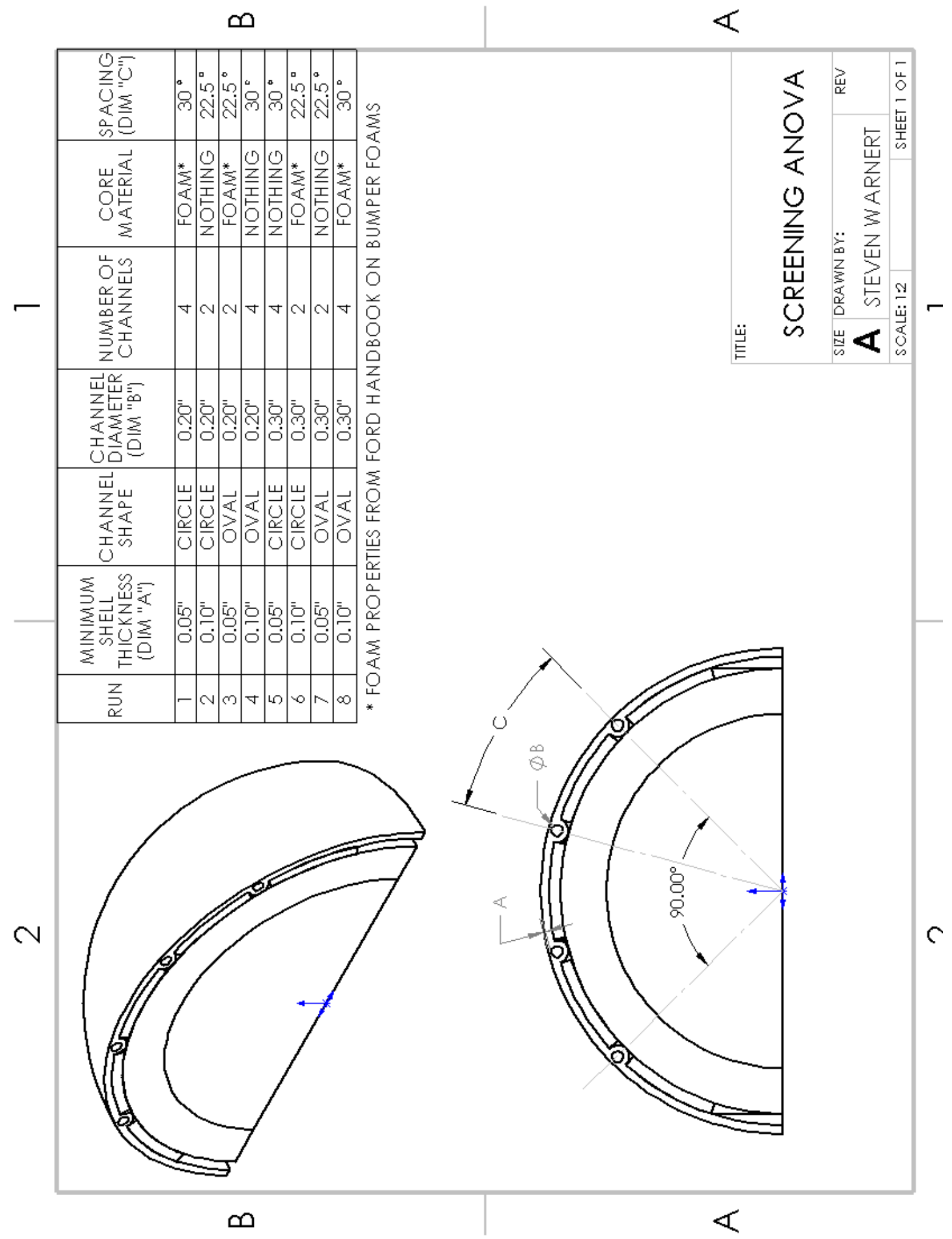




\section{Appendix G: Representative Matlab Code for Calculating Severity Index}

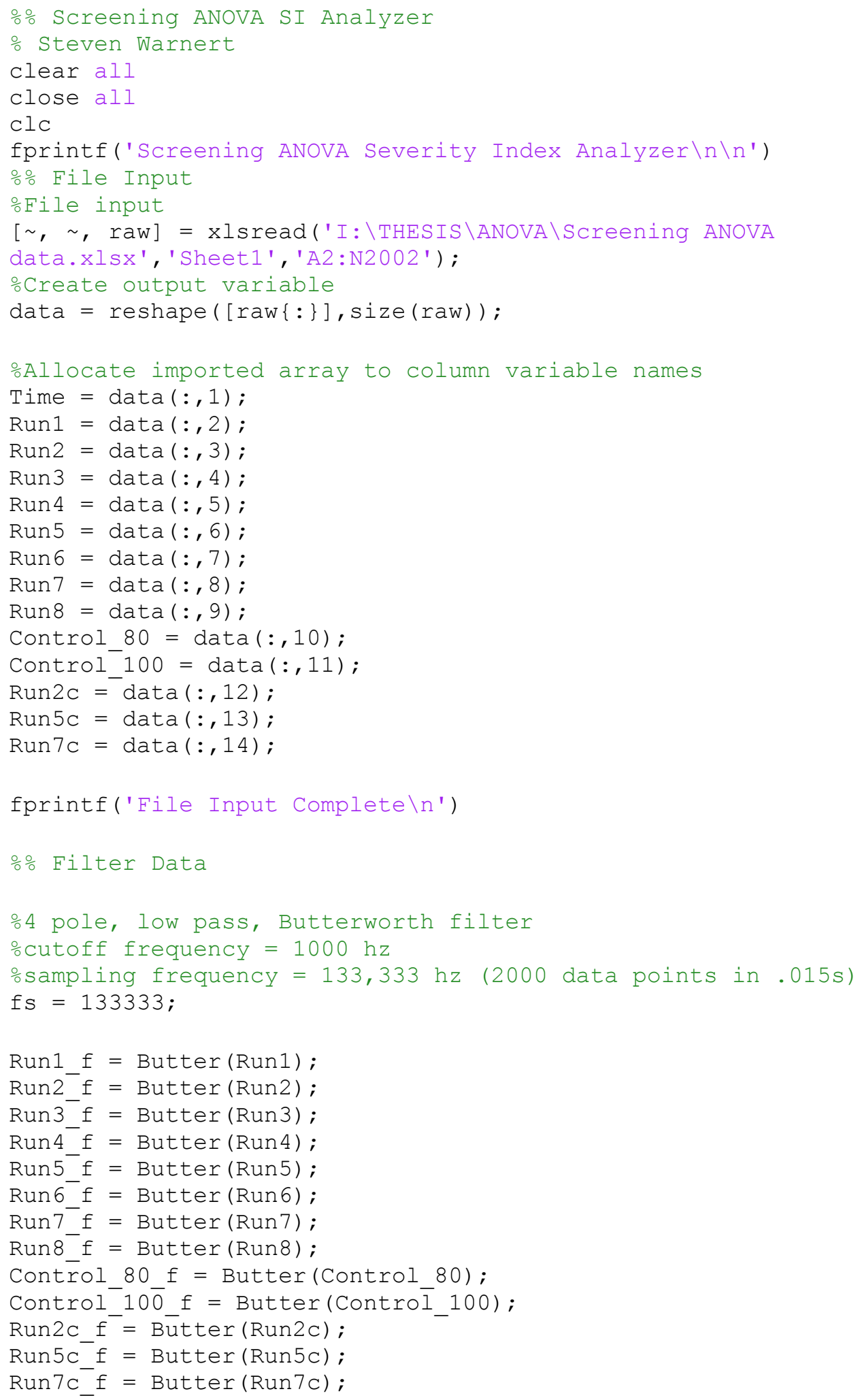




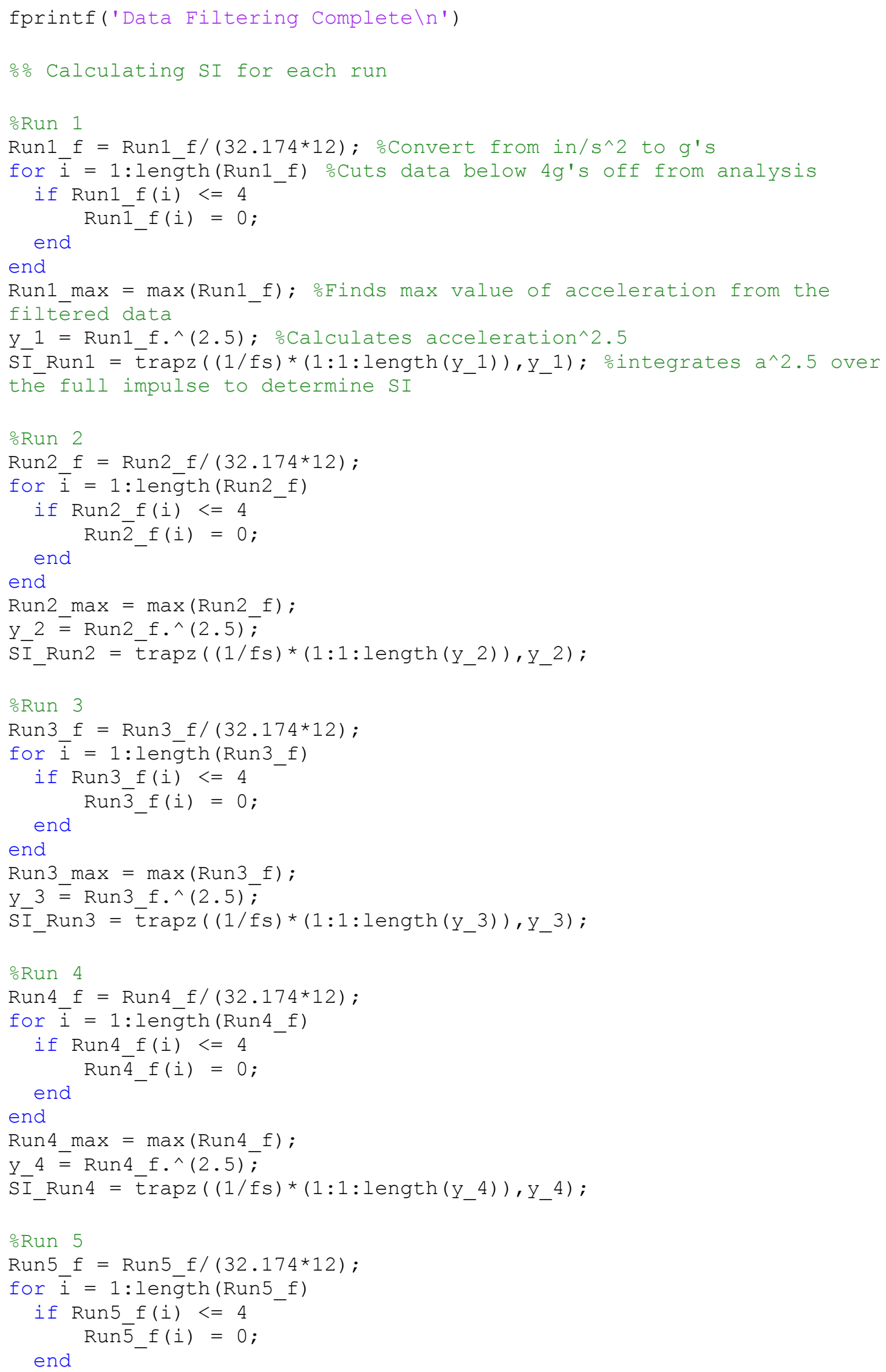




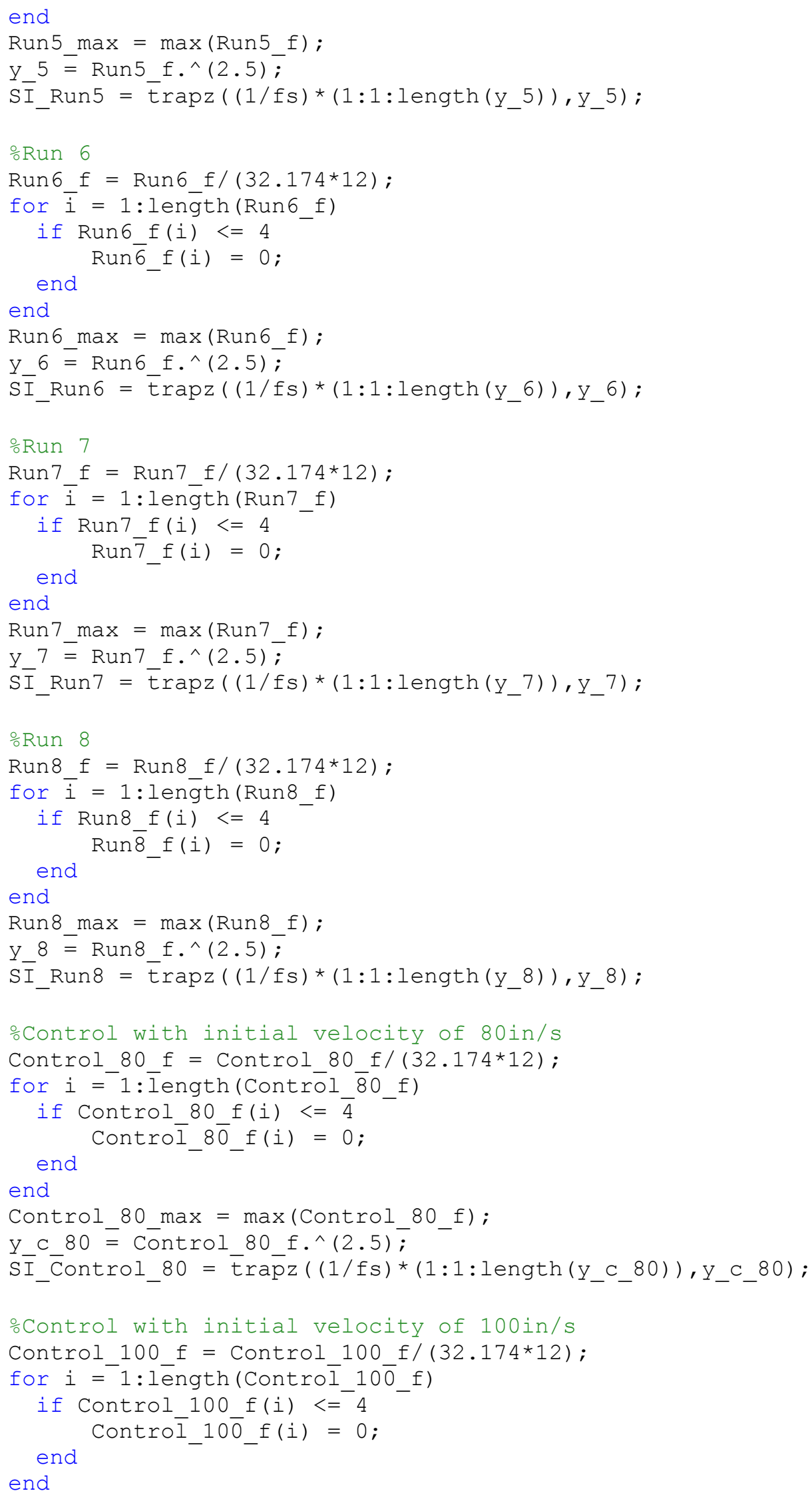




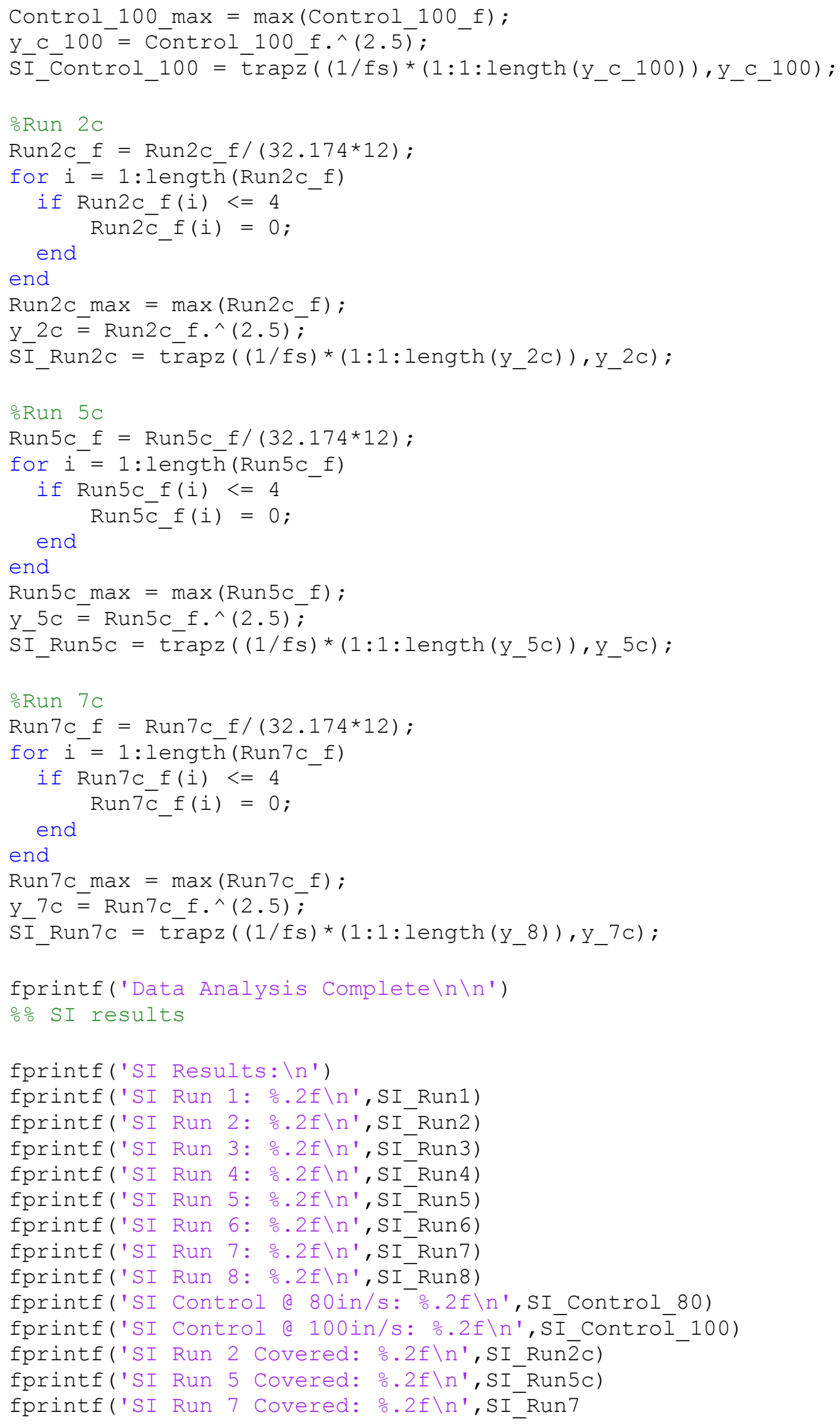




\section{Appendix H: Drawing of the Optimal Helmet Configuration}

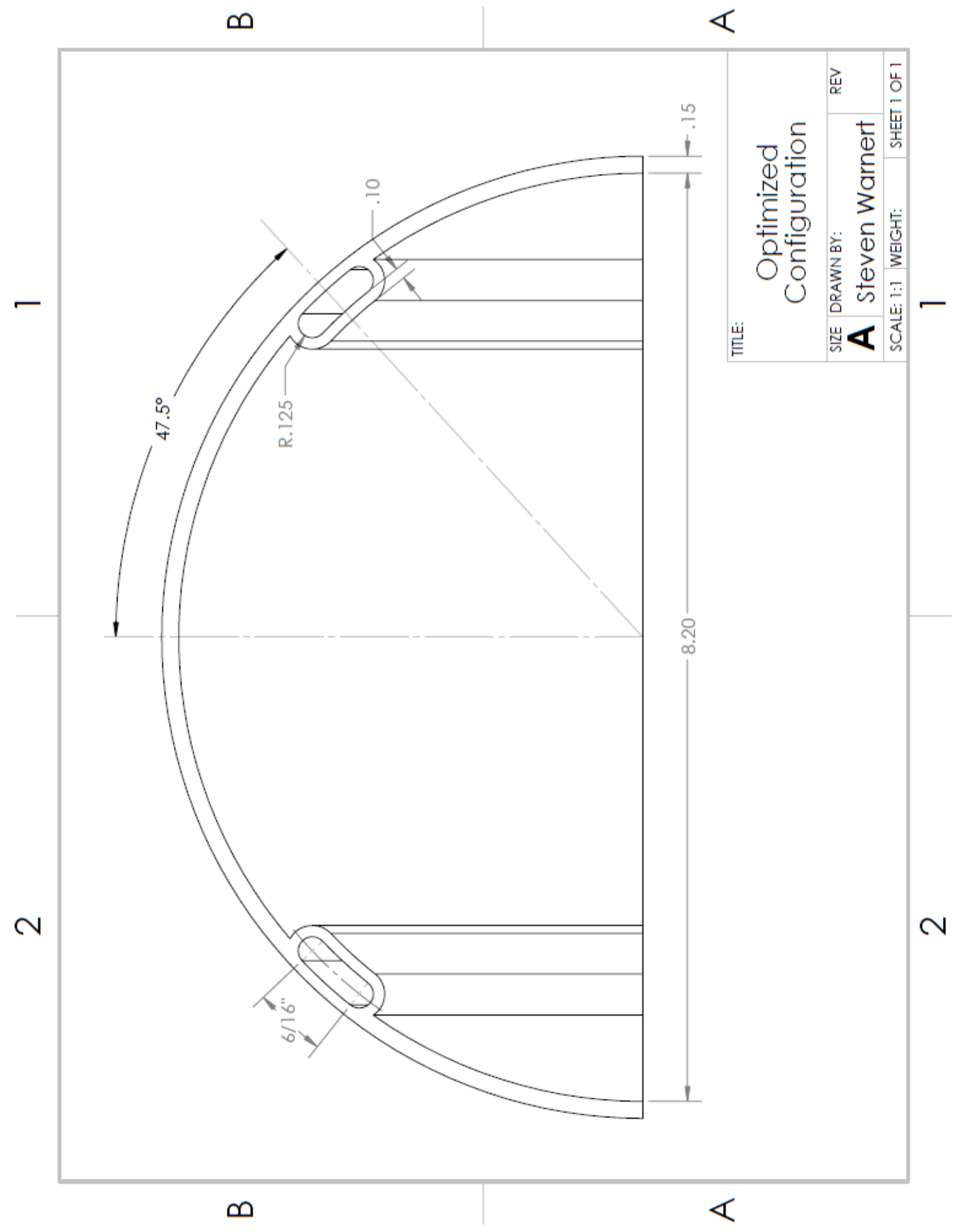




\section{Appendix I: High Speed camera screen captures of the first control helmet impact}

test

Images are given 13 frames $(2.6 \mathrm{msec})$ apart. Footage was filmed at 5000 frames per second.

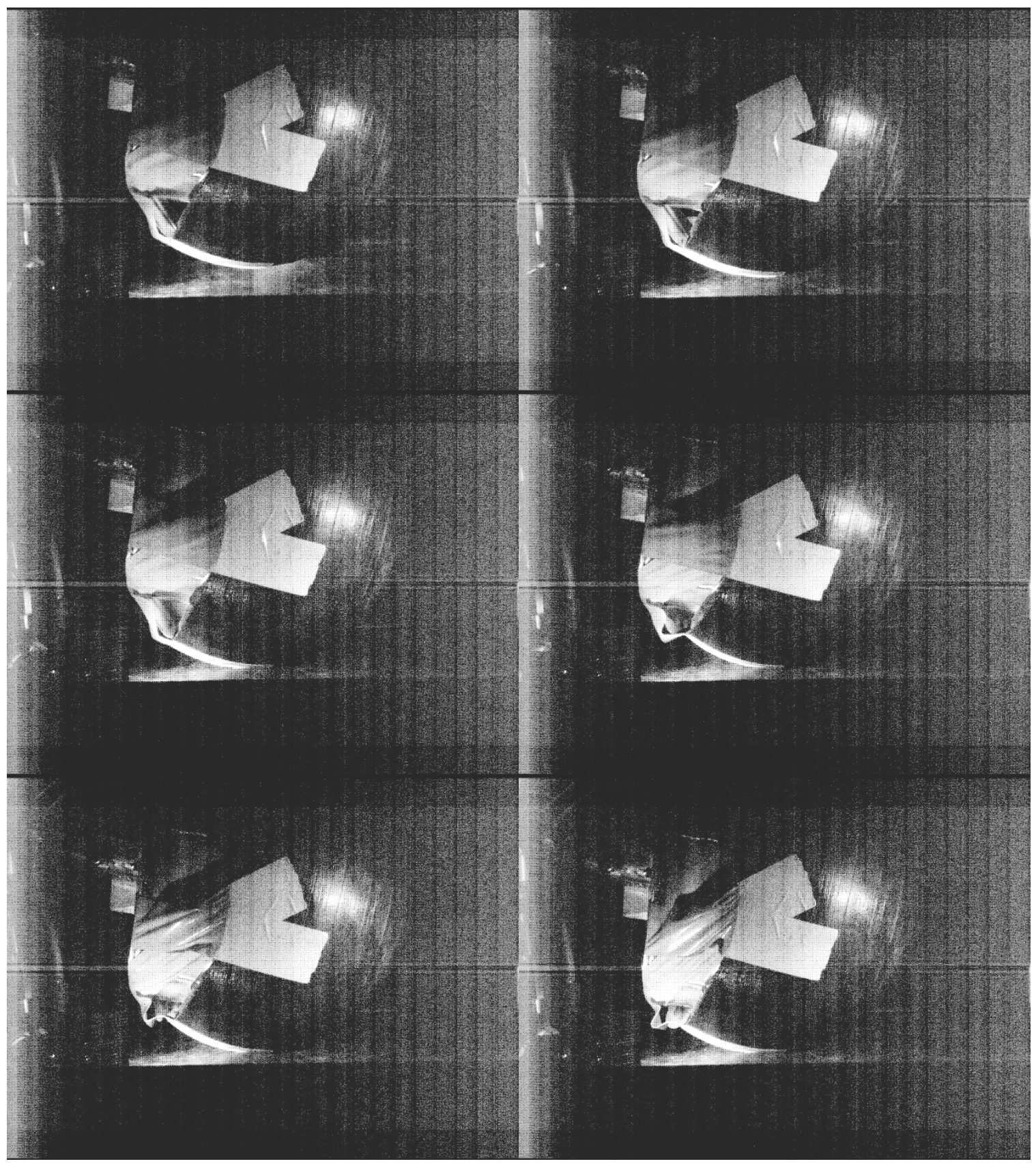




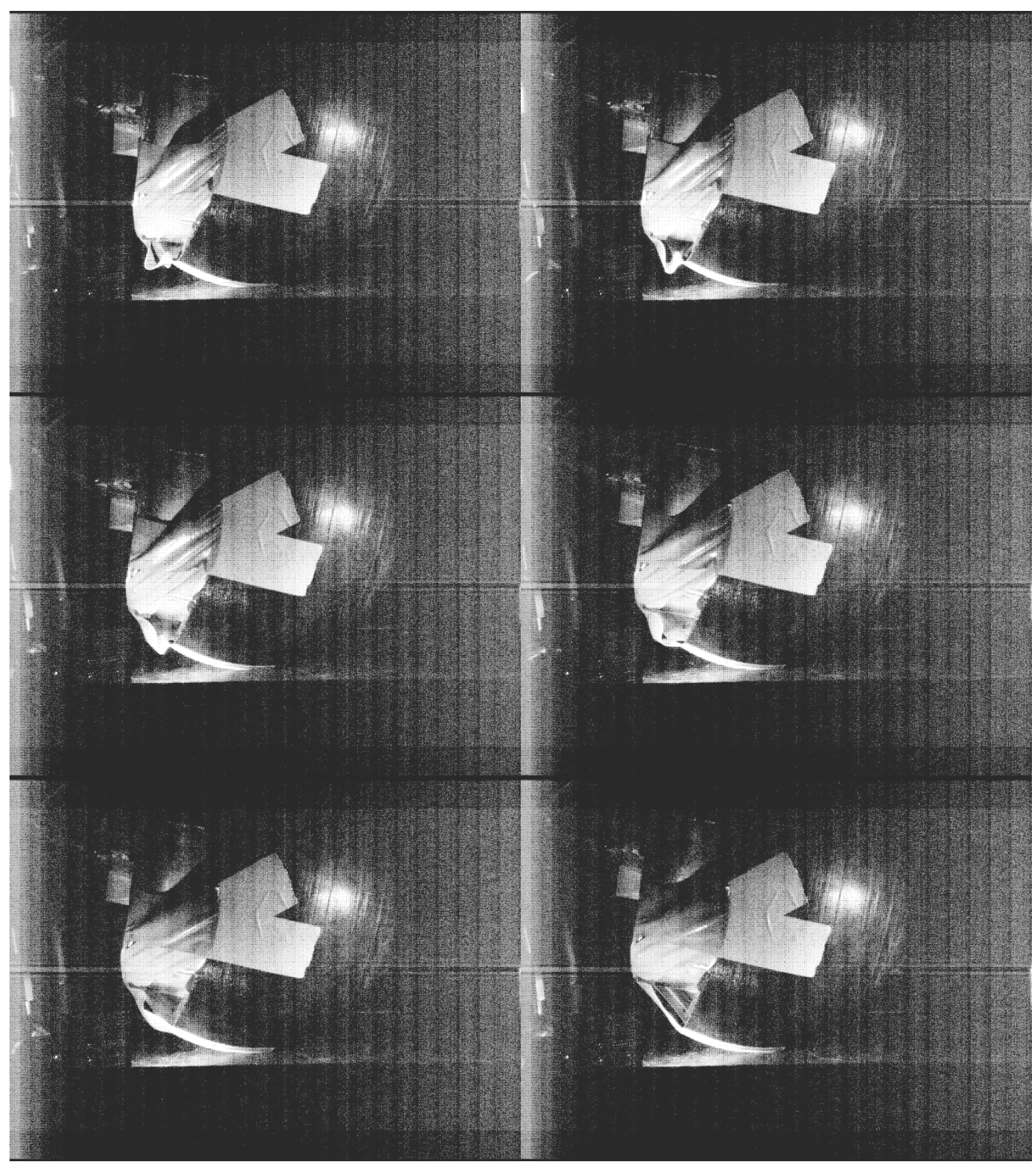

